\title{
The stapedius muscle of the rat : developmental aspects and adaptive properties of stapedius muscle fibre composition
}

Citation for published version (APA):

Dammeijer, P. F. M. (2008). The stapedius muscle of the rat : developmental aspects and adaptive properties of stapedius muscle fibre composition. [Doctoral Thesis, Maastricht University]. Datawyse / Universitaire Pers Maastricht. https://doi.org/10.26481/dis.20080117pd

Document status and date:

Published: 01/01/2008

DOI:

10.26481/dis.20080117pd

Document Version:

Publisher's PDF, also known as Version of record

\section{Please check the document version of this publication:}

- A submitted manuscript is the version of the article upon submission and before peer-review. There can be important differences between the submitted version and the official published version of record.

People interested in the research are advised to contact the author for the final version of the publication, or visit the DOI to the publisher's website.

- The final author version and the galley proof are versions of the publication after peer review.

- The final published version features the final layout of the paper including the volume, issue and page numbers.

Link to publication

\footnotetext{
General rights rights.

- You may freely distribute the URL identifying the publication in the public portal. please follow below link for the End User Agreement:

www.umlib.nl/taverne-license

Take down policy

If you believe that this document breaches copyright please contact us at:

repository@maastrichtuniversity.nl

providing details and we will investigate your claim.
}

Copyright and moral rights for the publications made accessible in the public portal are retained by the authors and/or other copyright owners and it is a condition of accessing publications that users recognise and abide by the legal requirements associated with these

- Users may download and print one copy of any publication from the public portal for the purpose of private study or research.

- You may not further distribute the material or use it for any profit-making activity or commercial gain

If the publication is distributed under the terms of Article $25 \mathrm{fa}$ of the Dutch Copyright Act, indicated by the "Taverne" license above, 
ISBN 9789052786872

- Copyright P.F.M. Dammeijer, Venlo 2008

Print and lay-out: Datawyse / Universitaire Pers Maastricht

Design of cover and lay-out: letje Thiery

Financial support; Abbott BV, Atos Medical BV, Artu Biologicals Europe, Beter Horen BV, Bioprof BV, CARA C'AIR BV, Entercare BV, GN Resound BV, GlaxoSmithKline BV, Ooms Allergie BV, Schering-Plough BV, Schoonenberg Hoorcomfort, Siemens Audiologie Techniek BV, VieCuri Medisch Centrum voor Noord-Limburg. 


\section{The Stapedius Muscle of the Rat}

Developmental aspects and adaptive properties of stapedius muscle fibre composition

\section{PROEFSCHRIFT}

ter verkrijging van de graad van doctor aan de Universiteit Maastricht

op gezag van de Rector Magnificus, Prof. mr. G.P.M.F. Mols,

volgens het besluit van het College van Decanen in het openbaar te verdedigen

op donderdag 17 januari 2008 om 14.00 uur

door

Patrick Fitzgerald Maria Dammeijer

geboren op 10 mei 1964 te Someren, Nederland

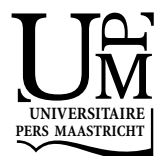




\section{PROMOTORES}

Prof. em. dr. J.J. Manni

Prof. em. dr. H. van Mameren

\section{COPROMOTOR}

Dr. L.J.C. Anteunis

\section{BEOORDELINGSCOMMISSIE}

Prof. dr. B. Kremer (voorzitter)

Prof. dr. P. van Dijk (Rijksuniversiteit Groningen)

Prof. dr. ir. J.H.M. Frijns (Universiteit Leiden)

Prof. dr. H. Kuipers

Prof. dr. A.F.M. Moorman (Universiteit van Amsterdam)

Financial support of the study by the Heinsius Houbolt Foundation and the Maastricht ENT Research Foundation, the Netherlands, is gratefully acknowledged. Publication and distribution of this thesis was financially supported by the Stichting Atze Spoor Fonds. 
Voor Petra

Floris, Maud en Joost 


\section{Table of Contents}

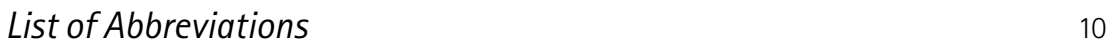

\section{CHAPTER 1}

Introduction. 11

1.1 Anatomy, physiology of the middle ear including the activity of the SM and TM 13

1.1.1 Anatomy; ossicular chain and middle ear muscles 13

1.1.2 Physiology: Conduction and modulation of sound in the middle ear 14

1.1.3 Activity of the SM 16

1.1.4 Activity of the TTM 16

1.1.5 The acoustic reflex 18

1.2 Supposed functions of the SM 19

1.2.1 Injury preventing theory 19

1.2.2 Interference theory 21

1.2.3 Desensitization theory 21

1.2.4 Ossicular-chain fixation theory 22

1.3 Remarks on the supposed functions of the SM 22

1.4 Aim and contents of this thesis 23

1.4.1 Fibre type composition of the adult SM 24

1.4.2 Postnatal changes of the fibre type composition in the SM 24

1.4.3 Electrophysiological measurement of the effect of different levels of noise exposure and sound deprivation 25

1.4.4 The effect of different exposures of sound on the fibre type composition of the SM 25

\section{CHAPTER 2}

Stapedius muscle fibre composition in the rat.

(Hear. Res. 2000; 141 (1-2), 169-179) 31

2.1 Introduction 33

$2.2 \quad$ Materials and Methods 34

2.2.1 Animals, dissection and tissue processing 34

2.2.2 mATPase characterization 35

2.2.3 MHC characterization $\quad 35$

2.2.4 Selection of muscle fibres 36

2.3 Results 37

2.3.1 EDL 37

$\begin{array}{lll}2.3 .2 & \text { SM } & 40\end{array}$

2.4 Discussion 42 
2.4.1 EDL characterization compared to previous EDL results 42

2.4.2 SM fibre composition 43

2.5 Conclusions 45

\section{CHAPTER 3}

Stapedius muscle fibre characterization during postnatal development in the rat. (Hear. Res. 2006; 219 (1-2), 48-55) 49

3.1 Introduction 51

3.2 Materials and Methods 52

3.2.1 Animals, dissection and tissue processing 52

3.2.2 mATPase characterization 53

3.2.3 MHC isoform characterization $\quad 54$

3.2.4 Selection of muscle fibres $\quad 54$

3.3 Results 54

3.4 Discussion $\quad 58$

3.5 Conclusions 60

\section{CHAPTER 4}

Effects of early auditory deprivation and stimulation on auditory brainstem responses in the rat. (Acta Otolaryngol. 2002; $122(7), 703-708)$

4.1 Introduction 67

4.2 Materials and Methods 69

$\begin{array}{lll}4.2 .1 & \text { Animals and procedures } & 69\end{array}$

$\begin{array}{ll}\text { 4.2.2 Electrophysiologic recordings } & 69\end{array}$

$\begin{array}{lll}4.2 .3 & \text { Data analysis } & 70\end{array}$

$\begin{array}{lll}4.3 & \text { Results } & 70\end{array}$

$\begin{array}{lll}4.3 .1 & \text { Thresholds } & 70\end{array}$

$\begin{array}{lll}\text { 4.3.2 Latencies } & 70\end{array}$

$\begin{array}{lll}4.4 & \text { Discussion } & 73\end{array}$

CHAPTER 5

Stapedius muscle fibre characterization in the noise exposed and auditory deprived rat. (Hear Res. 2007; 233 (1-2), 54-66) 79

5.1 Introduction 81

5.2 Materials and Methods 83

5.2.1 Animals and procedures 83

5.2.2 Muscle dissection and tissue processing 84

5.2.3 mATPase characterization 85 
5.2.4 MHC isoform characterization $\quad 85$

5.2.5 Selection of muscle fibres $\quad 85$

5.2.6 Statistical analysis 89

5.3 Results 89

5.3.1 Similarity of fibre composition in SMs of the same experimental group compared $\begin{array}{ll}\text { with differences between groups } & 89\end{array}$

5.3.2 Fibres with dominant expression of the MHC I isoform 90

5.3.3 mATPase type IIA fibres and expression of the MHC IIA isoform 90

5.3.4 mATPase type IIX fibres and expression of the MHC IIX isoform 90

5.3.5 The absence of fibres in a mATPase type IIB category 93

5.3.6 SMs only processed for mATPase activity staining after pre-incubation at $\mathrm{pH} 4.35 \quad 93$

5.4 Discussion 94

5.4.1 SM fibre's mATPase category assignment is able to predict its MHC isoform expression to a large extent $\quad 94$

5.4.2 Results obtained from SMs only processed for mATPase activity staining after preincubation at pH 4.35 support the differences found between SMs which were subject for complete analysis

5.4.3 SM fibres in the mATPase types 'Misc' are MHC isoform hybrids, and constitute the continuum of fibre types intermediate to the fibre types mATPase I, IIA and IIX

5.4.4 The fibres of auditory deprived SMs differ significantly from the SMs in the control group with respect to mATPase as well MHC typing

5.4.5 Noise exposed SMs versus control SMs show differences that are less apparent than $\begin{array}{ll}\text { auditory deprived SMs versus control SMs } & 97\end{array}$

$\begin{array}{lll}5.5 & \text { Conclusions } & 98\end{array}$

\section{CHAPTER 6}

$\begin{array}{ll}\text { General Discussion and Conclusions. } & 103\end{array}$

$\begin{array}{lll}6.1 & 104\end{array}$

6.2 Muscle fibre classification technique of the rat SM 104

6.2.1 The use of serial consecutive cross-sections to characterize the SM 104

$\begin{array}{lll}\text { 6.2.2 The choice of markers } & 105\end{array}$

6.2.3 Complementary information from mATPase classification and myosin heavy chain $\begin{array}{ll}\text { monoclonal antibodies } & 105\end{array}$

6.2.4 Expression of MHC cardiac- $\alpha$ isoform in the SM 106

6.2.5 IIB or not IIB? 107

6.2.6 Determination of neonatal MHC in the rat SM 107

$\begin{array}{lll}\text { 6.2.7 MHC isoform hybridism in the SM } & 108\end{array}$

6.3 Does the complex muscle fibre composition in the SM make sense? 108 
6.4 Muscle fibre characterization as a marker of maturation of the SM 110

6.5 Maturation of the rat SM runs parallel to and precedes that of the auditory system $\quad 110$

6.6 The effect of sound deprivation or noise exposure on ABR 111

6.6.1 Effect of early auditory deprivation on ABR in the rat 111

6.6.2 Effect of early noise exposure on ABR in the rat 112

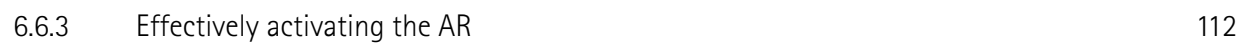

6.7 SM fibre characterization; its relevancy with respect to SM function 112

6.7.1 Injury-preventing function 112

6.7.2 Interference and desensitization theory 113

6.7.3 Function of the SM in the neonate rat versus the adult; is there a difference? 113

6.8 Implications for stapedius muscle related clinical issues $\quad 114$

6.8.1 Increased susceptibility to NIHL having a non-functional SM 114

6.8.2 Increased susceptibility to NIHL in the premature infant 114

6.8.3 Reconstruction of the SM tendon to the stapes prosthesis 115

$6.9 \quad$ Future research perspectives 116

$\begin{array}{lll}6.10 & \text { General Conclusions } & 117\end{array}$

\section{CHAPTER 7}

Summary and Summary in Dutch $\quad 123$

$\begin{array}{ll}\text { Summary } & 124\end{array}$

$\begin{array}{ll}\text { Samenvatting } & 126\end{array}$

$\begin{array}{ll}\text { CHAPTER } 8 & 129\end{array}$

$\begin{array}{ll}\text { Appendix 1. Clinical application of stapedius muscle contraction. } & 131\end{array}$

Appendix 2. Muscle fibre types; their classification and properties. 135

$\begin{array}{ll}\text { Acknowledgments in Dutch } & 147\end{array}$

Curriculum Vitae cover

List of Publications cover 


\section{List of Abbreviations}

\begin{tabular}{|c|c|}
\hline$A R$ & acoustic reflex \\
\hline$A B R$ & auditory brainstem responses \\
\hline ART & acoustic reflex threshold \\
\hline ATP & adenosine triphosphate \\
\hline $\mathrm{Cl}$ & cochlear implantation \\
\hline CM & cochlear microphonics \\
\hline CSA & cross sectional area \\
\hline$d E$ & decibel \\
\hline EDL & extensor digitorum longus \\
\hline EHC & enzymehistochemistry \\
\hline Emb & embryonal \\
\hline FG & fast glycolytic \\
\hline FOG & fast oxidative glycolytic \\
\hline GPD & $\alpha$-glycerophosphate dehydrogenase \\
\hline $\mathrm{IHC}$ & immunohistochemistry \\
\hline IPL & interpeak latency \\
\hline IR & immunoreactivity \\
\hline LDL & loudness discomfort level \\
\hline$m A b$ & monoclonal antibody \\
\hline mATPase & myofibrillar adenosine triphosphatase \\
\hline MEM & middle ear muscle \\
\hline MER & middle ear reflex \\
\hline Misc & miscellaneous \\
\hline $\mathrm{MHC}$ & myosin heavy chain \\
\hline Neo & neonatal \\
\hline NIHL & noise induced hearing loss \\
\hline PND & post natal day \\
\hline PTS & permanent threshold shift \\
\hline $\mathrm{SDH}$ & succinate dehydrogenase \\
\hline SL & sensation level \\
\hline SM & stapedius muscle \\
\hline SNHL & sensorineural hearing loss \\
\hline SO & slow oxidative \\
\hline SPL & sound pressure level \\
\hline TाM & tensor tympani muscle \\
\hline & temporary threshold shift \\
\hline
\end{tabular}




\section{Chapter 1}

\section{Introduction}

\section{Introduction}

te

is?

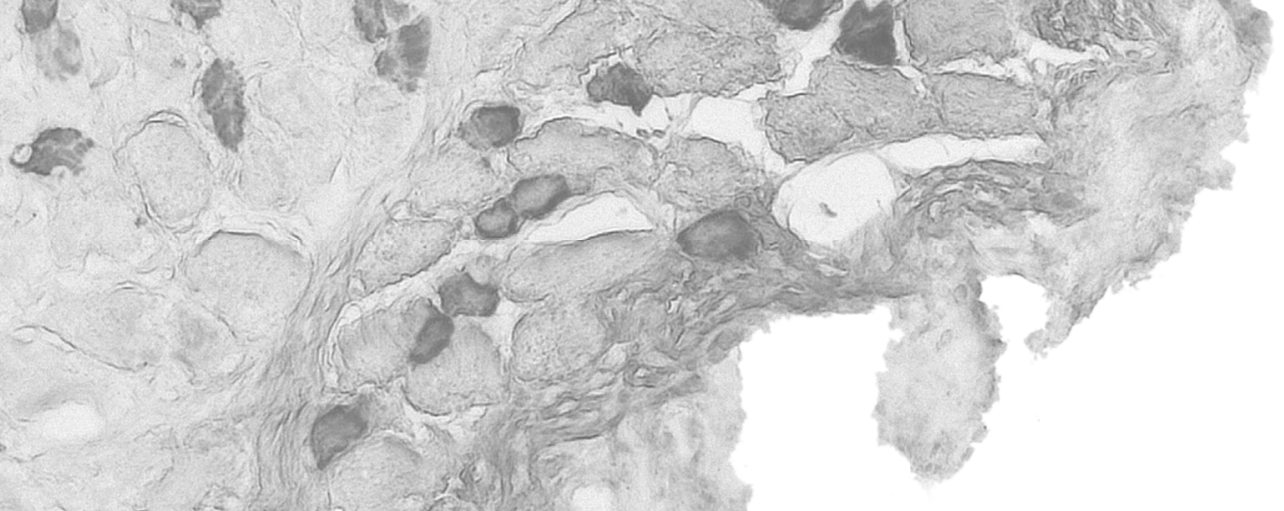

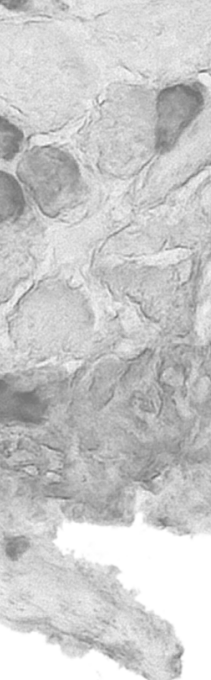

(biv 
Chapter 1 
The stapedius muscle (SM) is the smallest striated muscle in mammals and is located in the middle ear with its muscle tendon attached to the stapes. It is still a subject of debate whether the contracting SM modulates the acoustical energy in its way through the middle ear towards the cochlea. It is the aim of this thesis to contribute in the understanding of the function of the SM. In the studies presented in this thesis we investigated the adaptation of the SM muscle fibres to environments with varying acoustic exposition in order to further clarify the proposed function(s) of the SM.

Before describing the aims and design of our study, the anatomy of the middle ear, auditory system and proposed functions of the SM will be addressed in the following paragraphs. Moreover, we also describe the function of the tensor tympani muscle (TTM) although not being part of this thesis, because it is the other muscle situated in the middle ear besides the SM.

\subsection{Anatomy, physiology of the middle ear including the activity of the SM and TTM}

\subsubsection{Anatomy; ossicular chain and middle ear muscles}

The middle ear of mammals (see Fig. 1.1) contains the tympanic membrane and three small bones (ossicles); the malleus, incus and stapes constituting the ossicular chain. The manubrium of the malleus is integrated with the tympanic membrane; the head of the malleus is suspended in the epitympanum by ligaments. The head of the malleus articulates with the incus, which long process is connected to the stapes. The short process of the incus is held in place by the posterior incudal ligament. The stapes is suspended in the oval window of the cochlea by a posterior and anterior ligament, the posterior being stiffest. The two middle ear muscles (MEMs), SM and TTM are attached to the ossicular chain. The SM inserts to the head of the stapes and originates from inside the pyramidal process. The TTM inserts to the manubrium of the malleus and originates from the medial wall of the middle ear near the entrance to the Eustachian tube. The SM is largely encapsulated by the bony walls of the pyramidal process. The abundant presence of hyaluronic acid in the endomysium (the fibrous tissue surrounding the muscle) of the SM (Hellstrom et al., 1994) facilitates movement of the SM tissue within his bony capsule.

Despite the differences in architecture of the middle ear of the rat and the middle ear in man, the biomechanical organisation of the ossicular chain and the MEMs seems to be similar which justifies comparative studies by using the rat as a model (van den Berge et al., 1990a). Van den Berge (1990a) gave a detailed description of the gross anatomy of the rat's equivalent of the middle ear, the bulla, based on a computerized three dimensional reconstruction using consecutive cross-sections (thickness $10 \mu \mathrm{m}$ ) of the whole bulla (see Fig. 1.2a-b for a 3-dimensional impression of the rat's middle ear). 


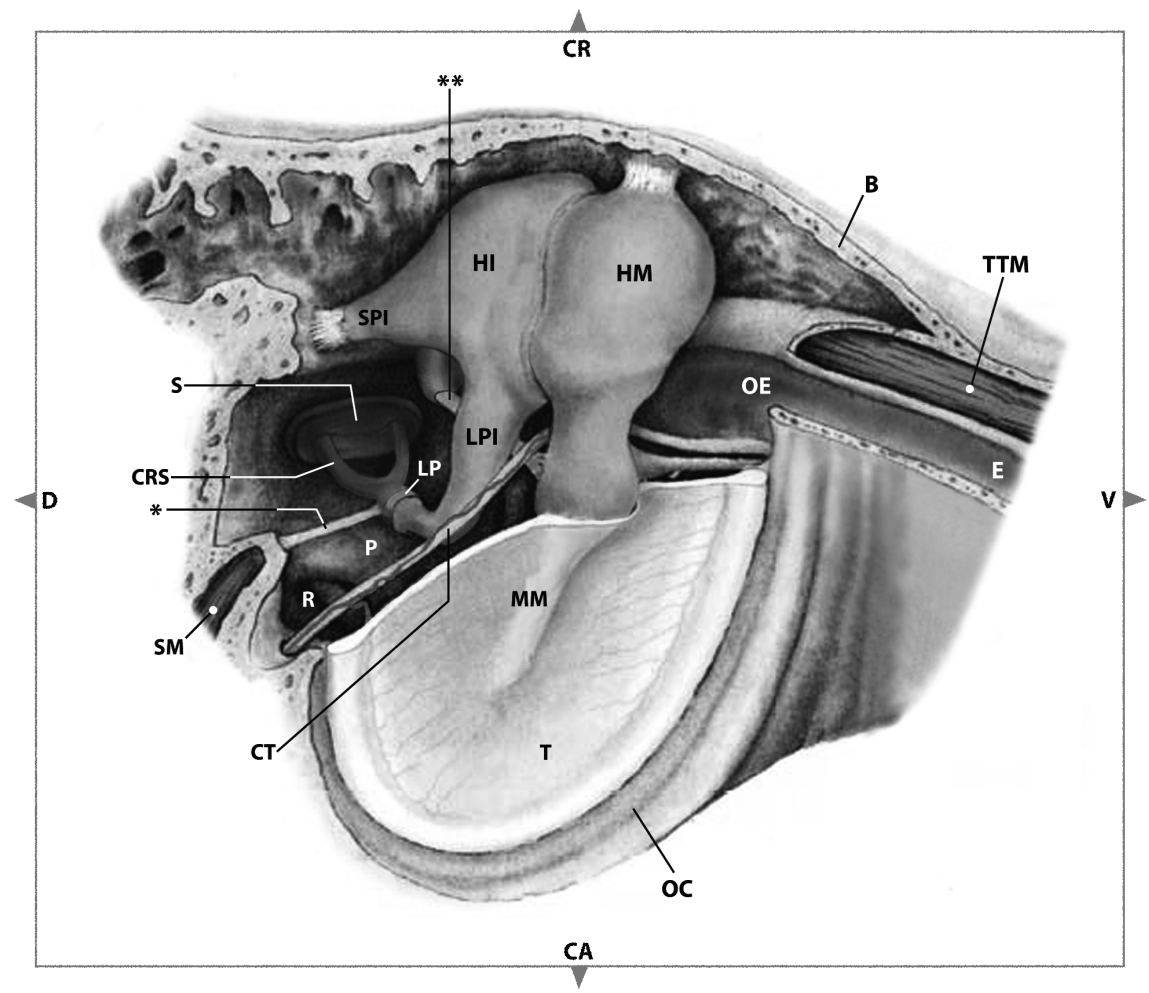

Fig. 1.1. Human right ear. Posterolateral view, pars flaccida of tympanic membrane and bone covering the epitympanic space laterally and posterior ear canal wall has been removed. $B$, bony wall of tympanic cavity; $C A$, caudal side; $C R$, cranial side; CRS, posterior crus of stapes; CT, chorda tympani nerve; $D$, dorsal side; E, Eustachian tube; HI, head of incus; HM, head of malleus ; LP, lenticulair process; $L P I$, long proces of incus; $M M$, manubrium of malleus; $O C$, outer ear canal; $O E$, tympanic orifice of Eustachian tube; $P$, promontory; $P P$, pyramidal process (opened); $R$, round window niche; $S$, stapes; SM, stapedius muscle; SPI, short process of incus; $T$, tympanic membrane; TTM, tensor tympani muscle; $V$, ventral side; ${ }^{*}$, tendon of SM; **, tendon of TTM (modified from Rauber and Kopsch, 1987).

\subsubsection{Physiology: Conduction and modulation of sound in the middle ear}

The tympanic membrane converts sound into vibration of the malleus. The vibration is then transmitted through the ossicular chain to the stapes footplate by which the fluid in the cochlea, or inner ear, is set into motion. The middle ear acts as a frequency dependent impedance transformer that converts vibration of air in the external acoustic meatus (low impedance) to vibration of fluid (high impedance) in the cochlea. This impedance matching action of the human middle ear is accomplished by the ratio between the surface area of the tympanic membrane and that of the stapes footplate and also by the lever action of the ossicles. Together, this leads to a gain of the sound pressure of approximately 25-30 dB at the footplate for the lower frequencies and less for frequencies above $1 \mathrm{kHz}$ (Aibara et al., 


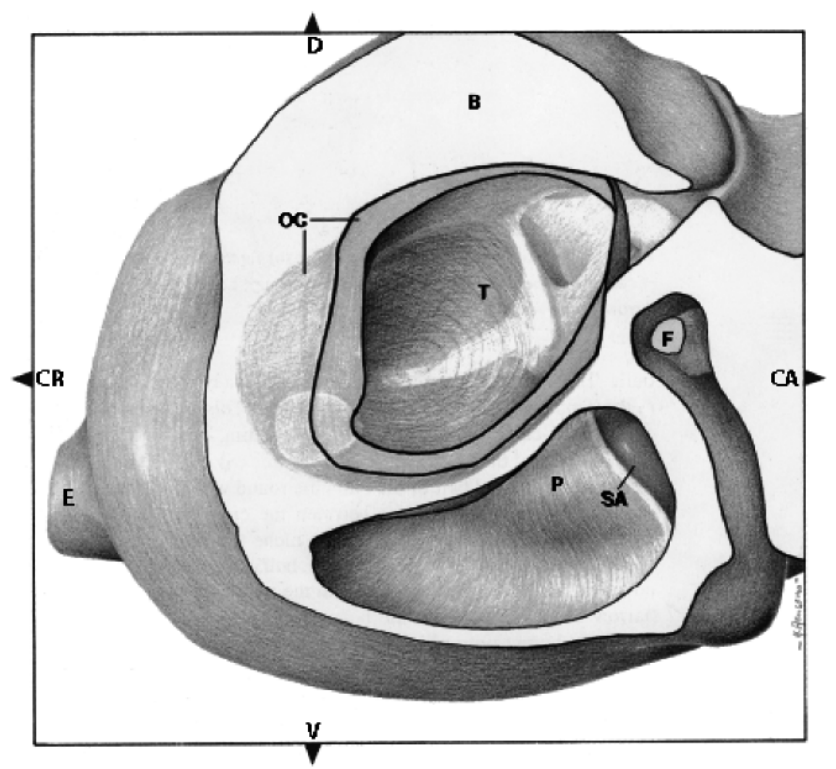

Fig.1.2a.

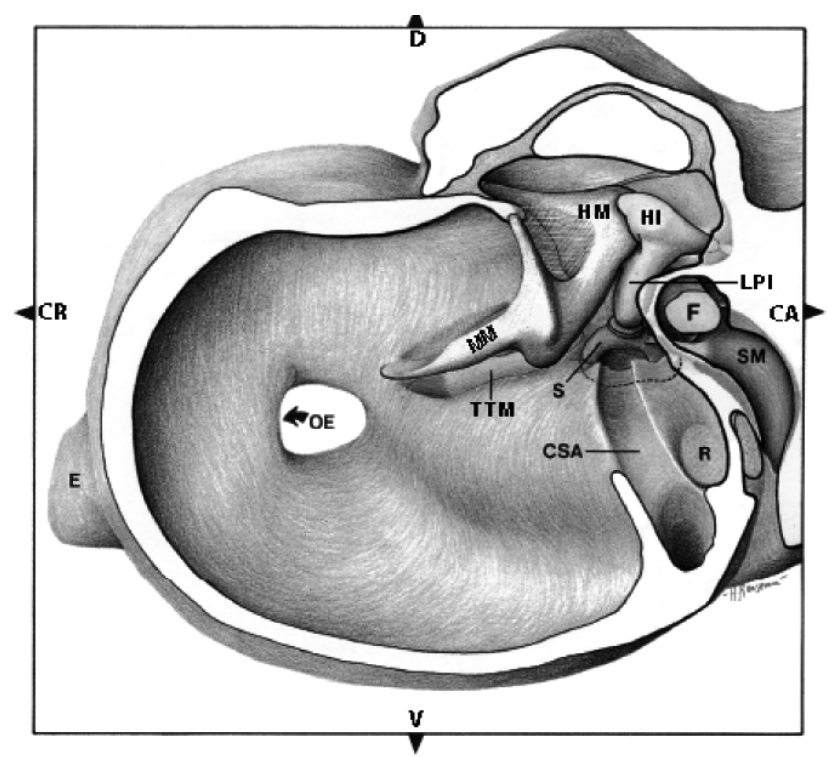

Fig. 1.26.

Legend to Figs. 1.2(a-b). Plastic representation of left tympanic bulla of the rat. Lateral views into the bulla. B, bony wall of tympanic bulla; CA, caudal side; CR, cranial side; CSA, canal of stapedial artery; D, dorsal side; E, Eustachian tube; F, facial nerve; HM, head of malleus; $H I$, head of incus; $L P I$, long proces of incus; $M M$, manubrium of malleus; $O C$, outer ear canal; OE, tympanic orifice of Eustachian tube; $P$, promontory; $R$, round window niche; $S$, stapes; $S A$, stapedial artery; $S M$, stapedius muscle; $T$, tympanic membrane; $T M$, tensor tympani muscle; $V$, ventral side. 


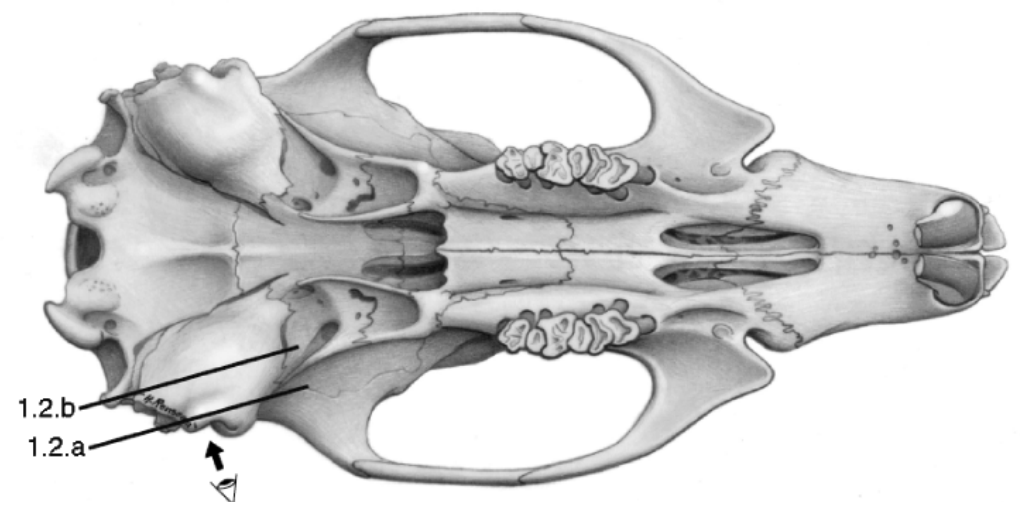

Fig. 1.2c. Ventral view on the base of the skull of the rat. The position of planes of sectioning through the tympanic bulla perpendicular to the lateral views into the bulla as is shown in Figs. $1.2(a-b)$ is indicated.

2001; Kurokawa and Goode, 1995). In delivering the enhanced vibration selectively to the oval window and not to the round window, a difference of force is created that effectively sets the fluids in the cochlea into motion.

\subsubsection{Activity of the SM}

The SM pulls the stapes in a direction that is perpendicular to its piston like motion (see Fig. 1.3), tilting the stapes so that it rotates around its posterior ligament and causes a sliding movement in the incudo-stapedial joint, parallel to its surface (Hüttenbrink, 1989). SM contraction causes a displacement of the stapes which impairs the usual piston-like motion of the stapes. This changes the way sounds are transmitted through the incudo-stapedial joint and leads to attenuation of transmission of sounds in the higher frequency range, for example in the cat up to $10 \mathrm{kHz}$ (Møller, 1984).

Other authors suggest that this attenuation is largest in the low-frequency range. During strong contractions, however, sound transmission is also reduced in the high-frequency range. In humans, by measuring the change in acoustic impedance of the middle ear, it was determined that reduction of sound transmission is largely limited to frequencies below $2 \mathrm{kHz}$ (Møller, 2000a). When measured by cochlear microphonics (CM) as was done in rats (Pang and Guinan, 1997), the attenuation is largest for $5 \mathrm{kHz}$ tones and with strong contractions the transmission is also reduced at $20 \mathrm{kHz}$.

\subsubsection{Activity of the TTM}

The TTM is reported to contract following exposure to bursts of loud noise. Hereby it contributes to an additional reduction of the ossicular vibration by the TTM contraction in some animal species (Møller, 1965). Howell (1984) believes that in humans the TM has no specific function. However, several other functions have been suggested by different authors. The TM would improve air exchange in the 


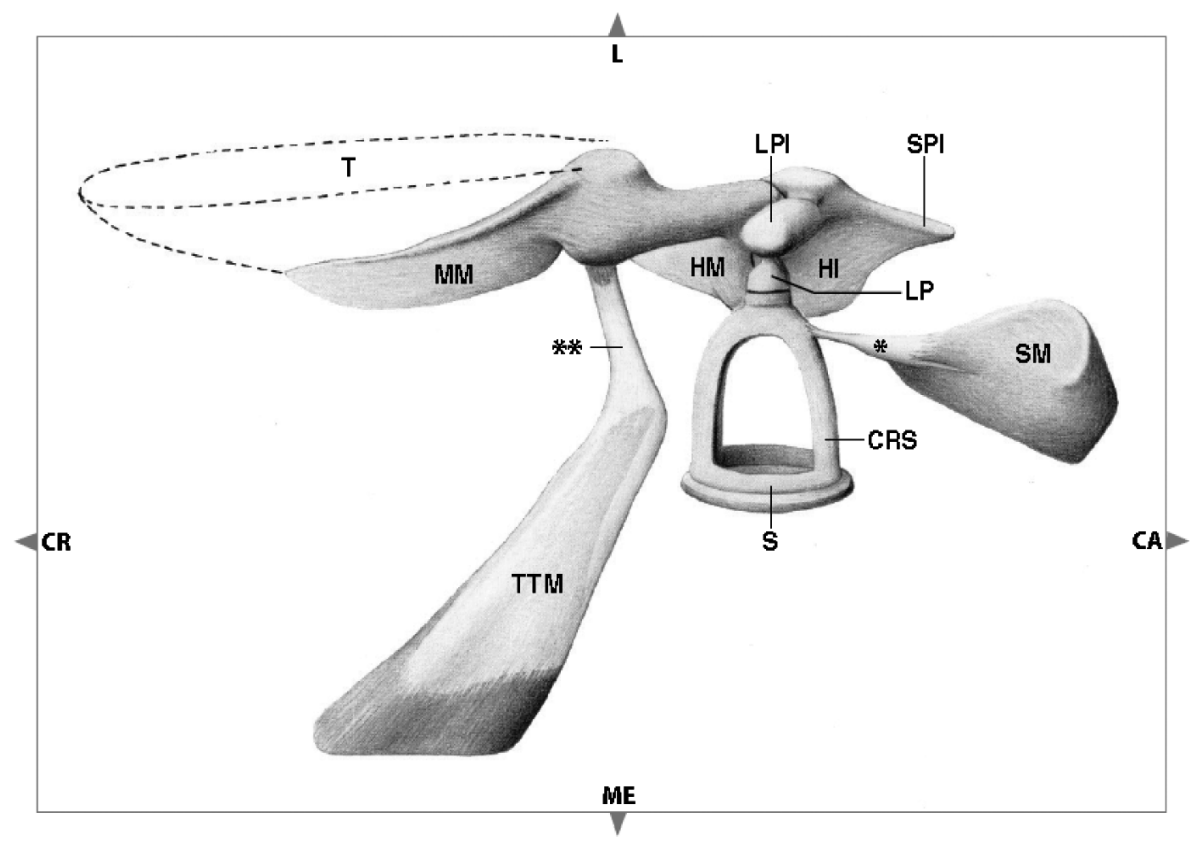

Fig. 1.3. Plastic representation of the ossicular chain, middle ear muscles and pars tensa of the tympanic membrane of the rat. Ventral view. $C A$, caudal side; $C R$, cranial side; $C R S$, posterior crus of stapes; $H M$, head of malleus; $H I$, head of incus; $L$, lateral side; $L P$, lenticulair process; LPI, long proces of incus; ME, medial side; MM, manubrium of malleus; S, stapes; SM, stapedius muscle; SPI, short process of incus; $T$, tympanic membrane; $T M M$, tensor tympani muscle; ${ }^{*}$, tendon of $S M_{;}^{* *}$, tendon of $T T M$.

middle cavity by contracting during swallowing, and therefore enhancing the opening of the Eustachian tube (Salen and Zakrisson, 1978). The TTM is also considered to be one of the effector organs of the startle reflex (Klockhoff and Anderson, 1959; Møller, 2000a; Mulder et al., 1993). Reaction of the TTM was elicited by nasally applied trigeminal stimulants (Rauchfuss et al., 1987). Also a ligament-like function has been suggested i.e. stabilizing the malleus, and thereby the tympanic membrane, in preventing a lateral movement of the malleus and tympanic membrane (Cavaliere et al., 2003; Hüttenbrink, 1988). The TTM is supposed to have a role in protecting the middle ear components, during sneezing, coughing, in which case there is a large air pressure increment from the pharynx through the Eustachian tube. TMM contraction may protect the middle and inner ear to mechanical damage caused by sudden pressure elevation in the external meatus (Cavaliere et al., 2003). The TM shows activity in respons to air jet directed into the orbit of the eye, unlike the SM, with long onset latency (Djupesland, 1975) and with variable amplitude (Greisen and Neergaard, 1975).

It is possible that the described functions of the tensor tympani muscle in humans are incomplete. 


\subsubsection{The acoustic reflex}

Contractions of the MEMs are part of the acoustic reflex (AR). This reflex occurs in response to exposure to a loud sound. The AR has been studied extensively in both humans and animals. Contractions of the SM and TTM result in stiffening of the middle ear bone chain, thereby altering the transmission of sound to the cochlea (Møller, 1961). In some animals, for example rabbits, both the SM and TM contract together routinely as an AR. (Borg, 1972). The SM is considered the main effector organ of the acoustic middle ear reflex in human (Møller, 1965; Brask, 1979) and the rat (Berge et al., 1990b; Pilz et al., 1997). The AR plays an important role in modern audiological testing (Møller, 2000a), see Appendix 1 for clinical applications. In humans, the AR is recorded non-invasively during tympanometry as a change of the ear's acoustic impedance (Zakrisson et al., 1974).

The AR can not be shown with tympanometry when there is a discontinuity or fixation of one of the components of the middle ear ossicular chain or when there is a perforation of the tympanic membrane. The AR is also usually absent when measured by tympanometry in facial nerve palsy patients where the SM is paralysed on the affected side. However, Stach et al. (1984) reported TTM activity as an acoustic reflex in a Bell's palsy patient, only ipsilaterally provoked, at levels above $105 \mathrm{~dB}$ sound pressure level (SPL), with variable amplitude, slow onset and fast decay; however, this phenomenon was considered to be part of a startle reflex.

The SPL at which the AR is evoked is the acoustic reflex threshold (ART). In humans with normal hearing, the ART is approximately $85 \mathrm{~dB}$ SPL for pure tones and approximately $75 \mathrm{~dB}$ SPL or lower for broad band noise (Møller, 1965). In an environment with complex noise, SM activation occurs at much lower sound levels. Activity of the SM was recorded below the ART when the ART evoking stimulus was preceded by another stimulus (Kawase et al., 1997; Neumann et al., 1996; Sesterhenn and Breuninger, 1976). A much lower ART level was recorded in adults as well as infants by using broad band noise ( $2.5 \mathrm{kHz}$ to $11 \mathrm{kHz}$ ) as the reflex elicitor with $50 \mathrm{~dB}$ SPL at the maximum spectrum, determining the ART by means of measuring the wideband shifts in admittance and energy reflectance of the tympanic membrane (Feeney and Keefe, 2001; Feeney and Sanford, 2005).

In humans, the strength of the SM contraction increases gradually with increasing stimulus intensity (Møller, 1965; Teig, 1973). Attenuation of sound may thus vary from $2 \mathrm{~dB}$ to as much as $30 \mathrm{~dB}$ (Sesterhenn and Breuninger, 1978).

In rats, sound attenuation induced by the AR increased and the latency of the AR decreased continuously with increasing SPL above the ART (Pilz et al., 1997).

During the $A R$, the change in sound transmission is greater than the measured change in acoustic impedance. This is explained by the decoupling of the cochlea from the middle ear in addition to adding stiffness to the ear due to SM contraction and displacement of the stapes (Møller, 1965).

The AR is driven by sounds in either the ipsilateral or contralateral ear, although slightly less sensitive for the latter. The AR is most provoked by external binaural sounds. This can be explained by the neuroanatomical auditory pathways. The AR has the first synaps in the ventral cochlear nucleus. Inputs from the two cochleas are distributed inhomogeneously across the stapedius motoneuron pool in the 
brainstem in such a way as to produce a segregation of function. In such a way that motoneurons in one brain stem region respond preferentially (or exclusively) to contralateral sound and motoneurons in other regions respond preferentially (or exclusively) to ipsilateral sound (McCue and Guinan, 1988). Crossed and uncrossed multisynaptic pathways then eventually end at the facial (VIIth cranial nerve) and trigeminal motonuclei (Vth cranial nerve) that innervate the SM and TTM respectively.

\subsection{Supposed functions of the SM}

Beatty (1932) believed that human MEMs have no active function to fulfil. Irvine and Wester (1974) showed that sound reaching the cochlea through bone conduction is attenuated by MEM contraction in cats and therefore it should be relevant in attenuating self-generated sounds. In more recent studies, different functions are proposed (e.g. see Borg et al., 1984).

Three major functions are attributed to the SM activity; they are incorporated in the injury-preventing, interference and desensitization theory as presented by Borg et al. (1984). Moreover, the MEMs are supposed to fulfil their role in the ossicular-chain fixation theory.

\subsubsection{Injury preventing theory}

Several studies showed the protective effect of the AR against high intensity acoustic stimuli which may cause noise induced hearing loss (NIHL) (Borg et al., 1983; Brask, 1977; Fletcher and Riopelle, 1960; Hilding, 1961; Kato, 1913; Miller et al., 1963; Sokolovski, 1973; Zakrisson, 1975; Zakrisson, 1979; Zakrisson et al., 1975; Zakrisson et al., 1980).

Several objections to the above mentioned NIHL protective function of the AR have evolved in literature the past decades. The latency of the AR was thought to be too long to protect effectively against impulse noise (Fletcher and Riopelle, 1960). By measuring the CM in the closed bulla in awake rats, and effectively measuring the effect of the AR in the same ear where the reflex was elicited, and thereby quantifying its effect on the transfer of the provoking stimulus itself, Pilz et al. (1997) found very short latencies of only 6-10 ms. They stated that the AR is one of the fastest reflexes in mammals. This supports the protection hypothesis.

Also, the AR is supposed to fatigue if the ear is exposed to sound over a longer period of time. This is especially true when a loud pure tone is produced. However, in normal hearing human subjects, who were monaurally exposed to 30 minutes of tape recorded shipyard noise at $97 \mathrm{~dB}$ SPL (which was characterized by a variable temporal structure) only a slight reflex fatigue was observed, together with a parallel shift of the stimulus-response curve (average $4 \mathrm{~dB}$ ) (Nilsson et al., 1980). If the applied sound fluctuates in frequency and intensity, and especially if there are brief pauses, there is little decay of the AR strength (Borg, 1980; Borg et al., 1984). Thus, it is believed that under real life situations (like working in a loud factory, or going to a rock concert) the magnitude of the AR does not diminish significantly during the exposure. 
Despite the absence of fatigue in real life, the attenuation is thought to be too small to account for an effective protection; only low frequencies are supposed to be attenuated, consequently no protection against high frequency noise is supposed to be offered. The protection from NIHL, for the higher frequencies, by the reduction of noise in the lower frequencies was however illustrated in a study by Nilsson and Borg (1983). Shipyard workers with a unilateral conductive hearing loss for the frequencies up to $2 \mathrm{kHz}$ showed substantial less NIHL than occurred in the normal contralateral ear. The amount of attenuation by the AR depends on stimulus intensity and frequency. Pilz et al. (1997) and Berge et al. (1990b) showed for all frequencies tested in rats that there was steady increase of attenuation with increasing intensity. Berge et al. (1990b) found the attenuation of $0.25-8 \mathrm{kHz}$ tone bursts up to $115 \mathrm{~dB}$ SPL is limited to a mean maximum of $15 \mathrm{~dB}$ SPL. The maximal attenuation was shown to occur at $1 \mathrm{kHz}$. Frequencies above $2 \mathrm{kHz}$ appeared to be the best elicitor of the $A R$ in the rat. Pilz et al. (1997) found that the amount of attenuation by the AR in rats was highest at $5 \mathrm{kHz}(20 \mathrm{~dB}$ at $120 \mathrm{~dB}$ SPL) and fell with rising frequencies, although at $10 \mathrm{kHz}$ and $20 \mathrm{kHz}$ the attenuation still was around $18 \mathrm{~dB}$ and $5 \mathrm{~dB}$ respectively.

The threshold curves of hearing and of the AR run in parallel in humans (Møller, 1962; Jepsen, 1951). Data from research by Pilz et al. (1997) showed that the same is true for the rat. In the rat the ART was lowest at $10 \mathrm{kHz}$ (77 dB SPL) where the rat hears best. In earlier research the ART was suggested to be in the range of 2.5-3.5 kHz (Berge et al., 1990b; Murata et al., 1986). Thus although the AR seems to be more effective at lower frequencies, the AR can be elicited with a lesser intensity in a higher frequency range with still a significant attenuation of the incoming noise.

Some authors have questioned this NIHL preventing purpose of the SM because the kind of noise exposure leading to NIHL is not common in nature. It would therefore not likely to have played a role in the phylogenetic development of the AR (Avan et al., 1992; Kollar, 1994; Møller, 2000a). However, it was pointed out by Borg that in humans, vocalization can produce sounds reaching up to 120-130 dB. A 1 second exposure of $130 \mathrm{~dB}$ SPL contains the same energy as a 15 minute exposure to industrial noise at $102 \mathrm{~dB}$ SPL which has been shown to cause a significant temporary threshold shift (TS) (Borg et al., 1984).

Many authors showed that the risk of developing NIHL is increased in the absence of a proper SM function, for example in Bell's palsy patients (Brask, 1979; Fletcher and Riopelle, 1960; Zakrisson, 1975; Zakrisson et al., 1975).

Some individuals seem to be more prone to acquire noise induced hearing loss (NIHL). They have an abnormal $A R$, which is characterized by decreased amplitude and a faster decay of the AR at supra threshold levels (Colletti and Sittoni, 1986; Houghton et al., 1988). As individual variability in SM fatigue was reported being large, Nilsson et al. (1980) also suggested an individual susceptibility in acquiring $\mathrm{NIHL}$ at least determined partly by the effectiveness of the SM mediated AR.

Moreover, the ability a person's AR to adapt to the protective role may determine this NIHL susceptibility (Henderson et al., 1993; Nilsson et al. 1980). Furthermore in an audiometric follow-up study extending 26 years, rock musicians having performed in more or less continuous sound levels over $85 \mathrm{~dB}$ SPL for 
more than 25 years, were reported to have relatively well-preserved hearing (Axelsson et al., 1995). These authors suggested this was the result of a more effective contraction of their trained stapedius muscles (Axelsson et al., 1995). Muscle training capability of the SM during long term noise exposure in humans was also suggested by Weidauer and Lenarz (1982), because they found that the SM reflex decay or SM fatigue, was absent in NIHL patients due to a permanent muscle activation by the chronic noise exposure.

Moreover patients with stapes prosthesis, hereby losing the SM function, may have a higher susceptibility to NIHL (Møller, 2000b). The SM may therefore be considered as preventing NIHL to a certain extent. Thus, the quality of SM's performance involving muscle strength, speed of contraction and endurance, seems to be relevant when considering some reported properties of the SM's activity in relation to the individual susceptibility of acquiring NIHL.

\subsubsection{Interference theory}

The contraction of the SM can decrease the masking of high-frequency signals by lowfrequency noise SM activity (i.e., the upward spread of masking). This results in a relatively better reception of high frequency signals (Borg et al., 1984; Pang and Guinan, 1997; Liberman et al., 1998). This effect of the SM seems particularly relevant when acknowledging that an activation of the SM was described to occur at SPLs at much lower sound levels than the usual measured ART (Feeney and Sanford, 2005; Kawase et al., 1997; Neumann et al., 1996; Sesterhenn and Breuninger, 1976; Simmons, 1959). Several authors consider the unmasking effect of the SM, by attenuating lower frequencies less than the more meaningful high frequencies, the most important function of the SM (Avan et al. 1992; Wormald et al., 1995; Pang and Guinan, 1997). In humans with inactivated SMs, a deterioration of discrimination of speech in noise (Liden et al., 1964) as well as speech at high sensation levels was reported (Borg and Zakrisson, 1975; Mahoney et al., 1979.). Also speech intelligibility in stapedectomized individuals is worse (Chadwell and Greenberg, 1979).

In phylogenetic context, development of the AR would suggest that its unmasking purpose is the most relevant, the SM being much better developed in animals having ultrasound hearing than those having better hearing for the lower frequencies (Kollar, 1994). In this respect it is interesting to note that bats have exceptionally large MEMs (Wever and Vernon, 1961).

\subsubsection{Desensitization theory}

The AR prevents overloading of the sensory receptors of the cochlea, thereby maintaining a good level of sensitivity. The AR in this theory acts as automatic volume control that keeps the mean level of sound reaching the cochlea within certain limits. The cochlea does not function properly at sound levels above $85 \mathrm{~dB}$ hearing threshold level; this seems to be related to the frequency selectivity of the basilar membrane which is greater at low sound levels than at high levels. Moreover the actions of the outer hair cells modulate the vibration amplitude of the basilar membrane and increase the cochlear frequency selectivity; this role is greatest at low intensities. Therefore, the frequency selectivity of the 
cochlea is decreased with increasing sound intensities above $60 \mathrm{~dB}$ hearing loss. Thus attenuation of loud acoustic signals by the AR increases sensitivity and frequency selectivity of the cochlea (Møller, 2000a). In Bell's palsy patients a decreased loudness discomfort level (LDL) (McCandless and Schumacher, 1979) and diminished speech discrimination in noise (Borg and Zakrisson, 1975; Chadwell and Greenberg, 1979; Wormald et al., 1995) was reported which illustrates the consequence of losing a proper AR. A decreased LDL was reported after stapedectomy in otosclerosis patients (Martin and Beck, 1975) and an increased susceptibility to NIHL was suggested (Møller, 2000b). Furthermore in reconstructing the SM tendon to the stapes piston during otosclerosis surgery and hereby reinstalling an $A R$, several authors have reported an improved signal to noise ratio improving discrimination of speech in noise, better discrimination of loud speech and a higher LDL in comparative studies (Arnold et al., 2007; Causse et al., 1997; Colletti et al., 1993; Colletti and Fiorino, 1994; Gros et al., 2000; Rasmy, 1986).

\subsubsection{Ossicular-chain fixation theory}

The MEMs should serve to maintain the ossicles in their proper position. Magnus (1861) and Von Helmholz (1868) (cited by Borg et al., 1984) already stated that the TM serves to prevent excessive movement of the ossicles. Von Bekesy (1936) (cited by Borg et al., 1984) hypothesized, that the SM serves to prevent disarticulation of the incudo-stapedial joint at high-intensity sound levels with consequent distortion of sound perception. More recently the fixation theory is still advocated by some authors, for example Hüttenbrink (1988) stated that MEM action serves to control the position of the ossicles for optimal sound transmission.

Hüttenbrink (2007) stated that surgery for otosclerosis, when the stapes is replaced and the SM is cut, abnormally reduces the stiffness of the tympano-ossicular system. This abnormal ossicular chain laxity is said to be limited by preservation of the stapedius tendon, which gives a significant degree of stiffness on the conductive system, and to some extent it prevents dislodgement of the stapes prosthesis either out the footplate or too far into the scala vestibuli causing vertigo during sneezing or loud noise (Colletti et al., 1993).

\subsection{Remarks on the supposed functions of the SM}

In discussing the theories concerning the functions attributed to the MEMs it is important to acknowledge what is actually meant by the terms function and purpose. Is function confined to the primary effect of the muscles' activity on sound transmission? Or is meant the effect on a higher integrated level as the influence of speech perception in noise for example? Also in discussing the function or purpose of the MEMs in some papers, the phylogenetic context is taken as an argument. This context contains regarding the SM having survival value in a species specific manner. This may be illustrated by the species specific important value of the SM in echo-locating bats (Suga and Jen, 1975). We use the meaning of function or purpose in relation to the SM in the context of having a certain effect 
on the auditory perception. Not only by constituting an acoustic attenuating input filter for the cochlea, but also the effect of the SM activity on frequency sensitivity and selectivity, and as a consequence on the quality of perception of perhaps meaningful signals in background noise. Although the SM may have evolved in man or rat for example for one purpose, it may also serve other functions in certain environments.

An example of what by some (Avan et al. 1992; Kollar, 1994) may be considered a side effect of the SM activity is offering some degree of protection from NIHL in workers exposed to industrial noise, or in musicians performing in a rock group. We suggest that the SM may adapt to the exposure of specific levels of sound to the middle ear.

\subsection{Aim and contents of this thesis}

Most of the supposed functions of the SM are related to the presentation of sound. Therefore we expect that exposing rats to noise or in contrast, deprive them from sound will show a corresponding change of the level of activity of the SM. A tuning of the SM muscle fibre characterization, i.e. performance, towards a direction that is similar to occur in skeletal muscles (Pette, 2002) in training or unloading experiments will may explain the functional relevance of the SM.

At present it is unknown whether the SM is susceptible to muscle training effects by sound exposure and if its muscle fibre composition does show changes comparable as is described to occur in skeletal muscles.

In skeletal muscles, interventions that unload the muscle cause slow-to-fast myosin heavy chain (MHC) conversions, whereas fast-to-slow conversions are seen when the muscles are engaged in resistance training and endurance exercise. We suggest that the function(s) of the SM in animals and humans may be clarified by studying the biochemical properties (fast and slow qualities) of their fibres as is stated decades ago (Borg et al., 1984). Characterization of the MHC isoform contents of the SM fibres may enable us to obtain a better insight into the functional purpose of this tiny muscle. Enzymehistochemistry $(\mathrm{EHC})$ and immunohistochemistry $(\mathrm{IHC})$ based muscle fibre typing of the SM may be conclusive. These classification methods and the functional and dynamical aspects of muscle fibres in general are presented in Appendix 2.

No study has ever been reported on investigating SM fibre composition adaptation in response to a change of the acoustical load. Therefore, we conduct a study in the rat concerning SM's capability of exhibiting training effects displayed by alterations of its muscle fibre composition. The rat was chosen because a large number of relevant audiological studies and histochemical research on skeletal muscles of this animal is available in the scientific databases.

If we can show adaptations of the rat SM fibre composition to high or low noise levels that are comparable to the adaptations of skeletal muscles which are subjected to (endurance) training or muscle unloading experiments, it would be indicative that the SM is dynamically active in modifying external sound that 
reaches the cochlea. It supports the role for the SM in the matter of development of resistance to hearing loss from loud noise. Also the question concerning whether the SM function could be like that of a stretch receptor such as a muscle spindle (Lyon and Malmgren, 1982) in stead of a muscle with mechanical effect on the middle ear ossicles would be answered in favour of the latter if SM fibre composition adapts to changing mechanical demands.

If we hypothesize that the SM fibre type composition adapts to changing demands as is expected to occur in long term exposition to (potentially) harmful noise we should first know more about the normal SM fibre type composition and developmental associated fibre changes to establish the control values to which the experimental data can be compared with.

\subsubsection{Fibre type composition of the adult SM}

In the past the muscle fibres of the SM have been characterized in a variety of species (Burgener and Mayr, 1980; Lyon and Malmgren, 1982; Veggetti et al., 1982; van den Berge and Wirtz, 1989; Godfrey et al., 1990a; Godfrey et al., 1990b; de Jong et al., 1988; Vita et al., 1983). In each study the method of defining the fibre types was limited by the accepted standard of techniques present at that time. Characterization of the SM fibre types has been performed by different methods; on the basis of their myofibrillar adenosine triphosphatase (mATPase) activity as well as on the basis of the expression of enzymes involved in specific types of ATP production. Only some used monoclonal antibodies (mAb) thought to be specific to a certain myosin heavy chain (MHC). This led to different numbers of a variety of muscle fibre types (see Appendix 2; Table A1).

Except for one more recent study (Jung et al., 2004), all studies use muscle fibre classification methods which do not comply with the complete MHC composition, especially since MHC IIX was recognized (Schiaffino et al., 1989; Sant'Ana Pereira et al., 1995). Thus, not knowing the MHC content of the SM and anticipating on the recent knowledge that MHC isoform co-expression occurs (van den Berge and Wirtz, 1989), necessitates performing MHC isoform typing of the normal rat SM first. We investigated the rat SM muscle fibre composition in the adult rat of which the results are presented in Chapter 2.

\subsubsection{Postnatal changes of the fibre type composition in the SM}

Developmental changes in skeletal muscle fibre composition are extensively reported in the literature concerning a diversity of mammalian skeletal muscles (see Appendix 2).

To investigate possible SM fibre composition changes caused by long term noise exposition or sound deprivation it is necessary to describe the normal (without noise exposition or sound deprivation) postnatal developmental changes of SM fibre composition. This has not been described before. Therefore we investigated the SM fibre type composition during postnatal development up to the 84th post natal day (= PND 84) in the rat. These results are presented in Chapter 3. 


\subsubsection{Electrophysiological measurement of the effect of different levels of noise exposure and sound deprivation}

To investigate the effects of sound deprivation and sound exposure on the SM fibre composition, two experimental and a control group of rats were included in the study. A bilateral conductive hearing loss was induced in the first experimental group by closing the external ear canal surgically. The second group was exposed to noise levels ranging between 65 and $90 \mathrm{~dB}$ SPL. The rats in the control group were kept at ambient noise levels.

Theoretically, changes in the SM fibre composition might also result from changes in auditory sensitivity as well as from changes in the maturation of neural parts of the stapedial reflex arc. Morphological changes of the central auditory nervous system have been described in the sound deprived mouse (Webster and Webster, 1997) and rats (Blatchley et al. 1983) and elevated noise stimuli can cause NIHL. Auditory Brainstem evoked Responses at supra-threshold levels revealed normal morphology in all rats. In the deprivation group the intended mild conductive loss was observed, in the noise exposed group no signs of a NIHL were found. A detailed description of these considerations and of the auditory findings in all three groups can be found in Chapter 4.

\subsubsection{The effect of different exposures of sound on the fibre type composition of the SM}

To investigate the effect of different exposures of sound on the fibre type composition of the SM, the sound deprivation and noise exposition began at PND 21. Several reasons for choosing this PND for starting the intervention exist. First, starting at the time of weaning was considered necessary in order to prevent nutritional and hence developmental aberration of the rat pups. Moreover, as the rat at birth does not have a functional auditory system, starting the experiment at a PND before a functional auditory apparatus exists would not serve the goal of testing the effect of different acoustic exposition on the muscle fibre composition of the SM. Furthermore in order to be able to compare the results between the different experimental groups the experiment started at the moment that the rats still had the same (short) history of noise exposition, the same auditory developmental stage and the same SM fibre composition as much as possible. Thus, the use of rats at this PND avoids the existence of individual differences in the fibre type composition of the SM which may perhaps have been developed already before even starting the experiment if we would have made use of rats at an older (adult) age. The effects of sound exposure and sound deprivation on the SM muscle fibre composition and comparisons with the SMs of rats held in an ambient noise environment are presented in Chapter 5.

In Chapter 6 the data and conclusions presented in prior chapters are discussed. 


\section{References}

- $\quad$ Aibara, R., Welsh, J.T., Puria, S., Goode, R.L., 2001. Human middle-ear sound transfer function and cochlear input impedance. Hear. Res. 152(1-2), 100-109.

- Arnold, A., Blaser, B., Hausler, R., 2007. Audiological Long-Term Results following Stapedotomy with Stapedial Tendon Preservation. Adv. Otorhinolaryngol. 65, 210-214.

- Avan, P., Loth, D., Menguy, C., Teyssou, M., 1992. Hypothetical roles of middle ear muscles in the guinea-pig. Hear. Res. 59(1), 59-69.

- Axelsson, A., Eliasson, A., Israelsson, B., 1995. Hearing in pop/rock musicians: a follow-up study. Ear Hear. 16(3), 245-253.

- Beatty, R.T., 1932. Hearing in man and animals. G. Bell and Sons Ltd. London.

- Berge, H. van den, Geest, A. van, Rensema, J.W., Drukker, J., 1990a. Three-dimensional graphic reconstruction of the tympanic bulla of the rat with special reference to the middle ear muscles. Acta Otolaryngol. 110(3-4), 253-261.

- $\quad$ Berge, H. van den, Wirtz, P., 1989. Detailed morphology of the stapedius muscle of the rat. J. Anat. 166, 157-169.

- Berge, H. van den, Kingma, H., Kluge, C., Marres, E.H.M.A., 1990b. Electrophysiological aspects of the middle ear muscle reflex in the rat: Latency, rise time and effect on sound transmission. Hear. Res. 48(3), 209-219.

- Blatchley, B.J., Williams, J.E., Coleman, J.R., 1983. Age-dependent effects of acoustic deprivation on spherical cells of the rat anteroventral cochlear nucleus. Exp. Neurol. 80, 81-93.

- Borg, E., 1972. Excitability of the acoustic m. stapedius an m. tensor tympani reflexes in the nonanesthetized rabbit. Acta Physiol. Scand. 85, 374-389.

- Borg, E., 1980. Recovery of the human intra-aural muscle reflex after "pauses" of various depth. Acta Otolaryngol. 90(1-2), 1-5.

- Borg, E., Counter, S.A., Rosler, G., 1984. Theories about middle ear muscle function. In: The acoustic reflex; Basic principles and clinical applications (Ed. S. Silman). Acad. Press, Orlando Florida, pp. 50-87.

- Borg, E., Nilsson, R., Engström, B., 1983. Effect of the acoustic reflex on inner ear damage induced by industrial noise. Acta Otolaryngol. 96, 361-369.

- Borg, E., Zakrisson, J.E., 1975. Stapedius muscle and speech perception. In: Sound perception in animals. Symposia of the Zoological Society of London (Eds. R.J. Bench, A. Pye and J.D. Pye) No: 37, 51-68.

- Brask, T., 1979. The noise protection effect of the stapedius reflex. Acta Otolaryngol. Suppl. 360, 116-117.

- Burgener, J., Mayr, R., 1980. Guinea pig stapedius muscle. A histochemical, light and electron microscopic study. Anat. Embryol. $161,65-81$.

- Causse, J.B., Vincent, R., Michat, M., Gherini, S., 1997. Stapedius tendon reconstruction during stapedotomy: technique and results. Ear Nose Throat J. 76(4), 256-568.

- Cavaliere, F., Masieri, S., Conti, G., Antonelli, M., Pennisi, M.A., Filipo, R., Proietti, R., 2003. Effects of non-invasive ventilation on middle ear function in healthy volunteers. Intensive Care Med. 29(4), 611-614.

- Chadwell, D.L., Greenberg, H.J., 1979. Speech intelligibility in stapedectomized individuals. Am. J. Otol. 1(2), 103-108.

- Colletti, V., Fiorino, F.G., 1994. Stapedotomy with stapedius tendon preservation: technique and long-term results. Otolaryngol. Head Neck Surg. 111(3 Pt 1), 181-188.

- Colletti, V., Fiorino, F.G., Sittoni, V., Policante, Z., 1993. Mechanics of the middle ear in otosclerosis and stapedoplasty. Acta Otolaryngol. 113(5), 637-641. 
- Colletti, V., Sittoni, W., 1986. Noise history, audiometric profile and acoustic reflex responsivity. In Salvi, R.J., Henderson, D., Hamernik, R.P., Colletti, V., (Eds.) Basic and applied Aspects of Noise Induced Hearing loss. New York, Plenum Press, pp. 247269.

- Djupesland, G., 1975. Advanced reflex considerations. In: J.J. Jerger (Ed.), Handbook of Clinical Impedance Audiometry. American Electromedics Cooperation, Dobbs Ferry, New York, pp. 85-125.

- $\quad$ Feeney, M.P., Keefe, D.H., 2001. Estimating the acoustic reflex threshold from wideband measures of reflectance, admittance, and power. Ear Hear. 22(4), 316-332.

- Feeney, M.P., Sanford, C.A., 2005. Detection of the acoustic stapedius reflex in infants using wideband energy reflectance and admittance. J. Am. Acad. Audiol. 16(5), 278-290.

- $\quad$ Fletcher, J.L., Riopelle, A.J., 1960. The protective effect of the acoustic reflex for impulsive noise. J. Acoust. Soc. Am. 32, 401404.

- Godfrey, D.A., Judkins, R.F., Wiet, G.J., Parli, J.A., Ross, C.D., Rubin, A.M., 1990a. Enzymes of transmitter and energy metabolism in cat middle ear muscles. Otolaryngol. Head Neck Surg.103, 799-804.

- Godfrey, D.A., Wiet, G.J., Parli, J.A., Beranek, K.L., Ross, C.D., 1990b. Enzymes of transmitter and energy metabolism in rat middle ear and extraocular muscles. Hear. Res. 48(3), 187-194.

- Greisen, O., Neergaard, E.B., 1975. Middle ear reflex activity in the startle reaction. Arch. Otolaryngol. 101(6), 348-353.

- Gros, A., Zargi, M., Vatovec, J., 2000. Does it make sense to preserve the stapedial muscle during surgical treatment for otosclerosis? J. Laryngol. Otol. 114(12), 930-934.

- Hellstrom, S., Laurent, C., Yoon, Y.J., 1994. Distribution of hyaluronan in the middle and inner ear. A light microscopical study in the rat using a hyaluronan-binding protein as a specific probe. J. Otorhinolaryngol. Relat. Spec. 56(5), 253-256.

- Henderson, D., Subramaniam, M., Boettcher, F.A., 1993. Individual susceptibility to noise-induced hearing loss: an old topic revisited. Ear Hear. 14(3), 152-168.

- Hilding, D.A., 1961. The protective value of the stapedius reflex: An experimental study. Trans. Am. Acad. Ophtalmol. Otolaryngol. 65, 297-307.

- Houghton, J.M., Greville, K.A., Keith, W.J., 1988. Acoustic reflex amplitude and noise-induced hearing loss. Audiology 27(1), 42-48.

- Howell, P., 1984. Are two muscles needed for the normal functioning of the mammalian middle ear? Acta Otolaryngol. 98(3-4), 204-207.

- Hüttenbrink, K.B., 1989. Die Bewegung der Gehörknöchelchen durch die Mittelohrmuskel- Kontraktion. Laryngorhinootologie 68(11), 614-621.

- Hüttenbrink, K.B., 1988. Zur Fixationtheorie der Mittelohrmuskelfunction. Laryngol. Rhinol. Otol. (Stuttgart) 67, 404-411.

- Hüttenbrink, K.B., 2007. Clinical significance of stapedioplasty biomechanics: swimming, diving, flying after stapes surgery. Adv. Otorhinolaryngol. 65, 146-149.

- Irvine, D.R., Wester, K.G., 1974. Middle ear muscle effects on cochlear responses to bone-conducted sound. Acta Physiol. Scand. 91(4), 482-496.

- Jepsen, 0., 1951. The threshold of the reflexes of the intratympanic muscles in a normal material examined by means of the impedance method. Acta Otolaryngol. 39(5), 406-408. 
- Jong, F.I.C.R.S. de, Kingma, H., Wirtz, P., Berge, H. van den, Marres, E.H.M.A., 1988. Indications of a differentiated regulation of sound transmission by the middle ear muscles of the rat. Am. J. Otol. 9(1), 70-75.

- Jung, H.H., Han, S.H., Nam, S.Y., Kim, Y.H., Kim, J.L., 2004. Myosin heavy chain composition of rat middle ear muscles. Acta Otolaryngol. 124(5):569-573.

- $\quad$ Kato, T., 1913. Zòr Physiology der Binnenmuskeln des Ohres. Pflògers Arch. Ges. Physiol. 150, 569-625.

- Kawase, T., Hidaka, H., Takasaka, T., 1997. Frequency summation observed in the human acoustic reflex. Hear. Res. 108(1-2), $37-45$.

- Klockhoff, I., Anderson, A., 1959. Recording of the stapedius reflex elicited by cutaneous stimulation. Acta Otolaryngol. 50, 451-454.

- Kollar, A., 1994. Bemerkungen zur Schutzfunktion der Mittelohrmuskeln. Otorhinolaryngol. Nova 4, 261-263.

- Kurokawa, H., Goode, R.L., 1995. Sound pressure gain produced by the human middle ear. Otolaryngol. Head Neck Surg. 113(4), 349-355.

- Liberman, M.C., Guinan, J.J., 1998. Feedback control of the auditory periphery: anti-masking effects of middle ear muscles vs. olivocochlear efferents. J. Commun. Disord. 31(6), 471-482.

- Liden, G., Nordlund, B., Hawkins, J.E., 1964. Significance of the stapedius reflex for the understanding of speech. Acta Otolaryngol. Suppl. 188, 275-279.

- Lyon, M.J., Malmgren, L.T., 1982. A histochemical characterisation of muscle fiber types in the middle ear muscles of the cat, the stapedius muscle. Acta Otolaryngol. 94, 99-109.

- Mahoney, T., Vernon, J., Meikle, M., 1979. Function of the acoustic reflex in discrimination of intense speech. Arch. Otolaryngol. 105(3), 119-123.

- Martin G., Beck C., 1975. Untersuchungen über die Unbehaglichkeitsschwelle des stapedektomierten Ohres. Ein Beitrag zur Funktion des M. stapedius. Arch. Otorhinolaryngol. 209(2), 107-111.

- McCandless, G.A., Schumacher, M.H., 1979. Auditory dysfunction with facial paralysis. Arch. Otolaryngol. 105(5), 271-274.

- McCue, M.P., Guinan, J.J., 1988. Anatomical and functional segregation in the stapedius motoneuron pool of the cat. J. Neurophysiol. 60(3), 1160-1180.

- Miller, J.D., Watson, C.S., Covell, W.P., 1963. Deafening effects of noise on the cat. Acta Otolaryngol. Suppl. 176.

- Møller, A.R., 1961. "Bilateral Contraction of the Tympanic muscles in Man". Royal Institute of Technology (KTH), Div. of TelegraphyTelephony, Report No. 18, pp. 1-5. Speech Transmission Laboratory, Stockholm.

- Møller A.R., 1962. The sensitivity of contraction of the tympanic muscles in man. Ann. Otol. Rhinol. Laryngol. 71, 89-95.

- Møller, A.R. 1965, effect of tympanic muscle activity on movement of the eardrum, acoustic impedance, and cochlear microphonics. Acta Otolaryngol. 58, 525-534.

- Møller, A.R., 1984. Neurophysiological basis of the acoustic middle-ear reflex. In: The acoustic reflex; Basic principles and clinical applications (Ed. S. Silman). Acad. Press, Orlando Florida, pp. 25-31.

- Møller, A.R., 2000a. Hearing: Its Physiology and Pathophysiology, section III; Acoustic reflexes, Academic Press, San Diego, CA, pp. 343-370.

- Møller, A.R., 2000b. Hearing: Its Physiology and Pathophysiology, section IV; Disorders of the Auditory System and Their Pathology, Academic Press, San Diego, CA, pp. 390-392. 
- Moore, D.R., 1985. Postnatal development of the mammalian central auditory system and the neural consequences of auditory deprivation. Acta Otolaryngol. Suppl. 421, 19-30.

- Mulder, J.J., Vantrappen, G., Snik, A.F., Manni, J.J., 1993. The use of the startle reflex measurement in patients with various types of fixation of the ossicular chain. Scand. Audiol. 22(4), 257-260.

- Murata, K., Ito, S., Horikawa, J., Minami, S., 1986. The acoustic middle ear muscle reflex in albino rats. Hear. Res. 23(2), 169-83.

- Neumann, J., Uppenkamp, S., Kollmeier, B., 1996. Detection of the acoustic reflex below 80 dB HL. Audiol. Neurootol. 1(6), 359369.

- $\quad$ Nilsson, R., Borg, E., 1983. Noise-induced hearing loss in shipyard workers with unilateral conductive hearing loss. Scand. Audiol. 12(2), 135-140.

- Nilsson, R., Borg, E., Liden, G., 1980. Fatigability of the stapedius reflex in industrial noise. Acta Otolaryngol. 89(5-6), 433-439.

- Pang X.D., Guinan J.J., 1997. Effects of stapedius-muscle contractions on the masking of auditory-nerve responses. J. Acoust. Soc. Am. 102(6), 3576-3586.

- $\quad$ Pette, D., 2002. The adaptive potential of skeletal muscle fibers. Can. J. Appl. Physiol. 27(4), 423-448.

- Pilz, P.K.D., Ostwald, J., Kreiter, A., Schnitzler, H., U., 1997. Effect of the middle ear reflex on sound transmission to the inner ear of the rat. Hear. Res. $105,171-182$.

- Rasmy, E., 1986. Stapedius reflex after stapedectomy with preservation of the stapedius tendon. J. Laryngol. Otol. 100(5), 521527.

- Rauber, A. Kopsch, F., 1987. Anatomie des Menschen, Part III, Georg Thieme Verlag, Stuttgart.

- Rauchfuss, A., Hiller, E., Leitner, H., Wöllmer, W., 1987. Reaktion des M. tensor tympani ausgelöst durch nasal applizierte Trigeminusreizstoffe. Laryngol. Rhinol. Otol. 66(3), 131-132.

- Salen, B., Zakrisson, J.E., 1978. Electromyogram of the tensor tympani muscle in man during swallowing. Acta Otolaryngol. 85(5-6), 453-455.

- Sant'Ana Pereira, J.A.A., Haan, de A., Wessels, A., Moorman, A.F.M., Sargeant, A.J., 1995. The mATPase histochemical profile of rat type IIX fibres: Correlation with MyHC immunolabelling. Histochem. J. 27, 715-722.

- $\quad$ Schiaffino, S., Gorza, L., Sartore, S., Saggin, L., Ausini, S, Vianello, M., Gundersen, K., Lomo, T., 1989. Three myosin heavy chain isoforms in type 2 skeletal muscle fibres. J. Muscle Res. Cell Motil. 10, 197-205.

- Sesterhenn, G., Breuninger, H., 1976. The acoustic reflex at low sensation levels. Audiology 15(6), 523-533.

- Sesterhenn, G., Breuninger, H., 1978. On the influence of the middle ear muscles upon changes in sound transmission. Arch. Otorhinolaryngol. 221(1), 47-60.

- Simmons, F.B., 1959. Middle ear muscle activity at moderate sound levels. Ann. Otol. Rhinol. Laryngol. 68, 1126-1143.

- Sokolovski, A., 1973. The protective action of the stapedius muscle in noise-induced hearing loss in cats. Arch. Klin. Exp. Ohren Nasen Kehlkopfheilkd. 203(4), 289-309.

- Stach, B.A., Jerger, J.F., Jenkins, H.A., 1984. The human acoustic tensor tympani reflex. A case report. Scand. Audiol. 13(2), 9399.

- Suga N. and Jen P.H.S., 1975. Peripheral control of acoustic signals in the auditory system of echolocating bats. J. Exp. Biol. 62, 277-311.

- Teig. E., 1973. Differential effect of graded contraction of middle ear muscles on the sound transmission of the ear. Acta Physiol. Scand. 88, 382-391. 
- Veggetti, A., Mascarello, F., Carpene, E., 1982. A comparative histochemical study of fibre types in middle ear muscles. J. Anat. $135,333-352$.

- Vita, G., Muglia, U., Germana, G., Pennica, F., Carfi, F., 1983. Histochemical characteristics of rabbit stapedius muscle. Exp. Neurol. $81(2), 511-516$.

- Webster, D.B., Webster, M., 1977. Neonatal sound deprivation affects brain stem auditory nuclei. Arch. Otolaryngol.103, 392398.

- Weidauer, H., Lenarz, T., 1982. Verhalten des Stapediusreflexes bei akustischer Langzeitstimulation. Laryngol. Rhinol. Otol. 61(12), 674-677.

- Wever, E.G., Vernon, J.A., 1961. The protective mechanisms of the bat's ear. Ann. Otol. Rhinol. Laryngol. 70, 5-17.

- Wormald, P.J., Rogers, C., Gatehouse, S., 1995. Speech discrimination in patients with Bell's palsy and a paralysed stapedius muscle. Clin. Otolaryngol. Allied Sci. 20(1), 59-62.

- Zakrisson, J.E., 1975. The role of the stapedius reflex in poststimulatory auditory fatigue. Acta Otolaryngol. 79(1-2), 1-10.

- Zakrisson, J.E., 1979. The effect of the stapedius reflex on attenuation and poststimulatory auditory fatigue at different frequencies. Acta Otolaryngol. Suppl. 360, 118-121.

- Zakrisson, J.E., Borg, E., Liden, G., Nilsson, R., 1980. Stapedius reflex in industrial impact noise: fatigability and role for temporary threshold shift (TS). Scand. Audiol. Suppl. 12, 326-334.

- Zakrisson, J.E., Borg. E., Diamant. H., Miller, A.R., 1975. Auditory fatigue in patients with stapedius muscle paralysis. Acta Otolaryngol. 79(3-4), 228-232.

- Zakrisson, J.E., Borg, E., Blom, S., 1974. The acoustic impedance change as a measure of stapedius muscle activity in man. A methodological study with electromyography. Acta Otolaryngol. 78(5-6), 357-364. 


\section{Chapter 2}

\section{Stapedius muscle fibre composition in the rat}

Patrick F.M. Dammeijer ${ }^{1}$, Henk van Mameren², Paul van Dijk², Antoon F.M. Moormann ${ }^{3}$, Petra Habets ${ }^{3}$, Johannes J. Manni' and Jan Drukker².

'Dept of Otorhinolaryngology and Head and Neck Surgery, University Hospital Maastricht ${ }^{2}$ Dept of Anatomy/Embryology, University Maastricht

${ }^{3}$ Dept of Anatomy/Embryology, University of Amsterdam

Published in Hearing Research 2000, 141(1-2), 169-179. 


\begin{abstract}
The stapedius muscle (SM) is supposed to prevent cochlear damage by noise. Consequently functional demands are the ability of fast contraction with long endurance. This implies the presence of a large fraction of myosin type II fibres with an appreciable oxidative capacity. We determined the myosin composition of SM fibres using consecutive complete SM cross-sections (6 weeks old rats) which were processed by enzymehistochemistry $(\mathrm{EHC})$ to determine acid/alkali lability of myofibrillar adenosine triphosphatase (mATPase) or by immunohistochemistry (IHC) using myosin heavy chain (MHC) antibodies. Method accuracy was determined in co-processed extensor digitorum longus (EDL). Four hundred SM and 200 EDL fibres were assigned to mATPase type I, IIA, IIB, IIX or 'Miscellaneous' ('Misc') categories. Per mATPase category the fibres were attributed to groups with specific MHC composition. In the EDL, mATPase type I and IIB fibres expressed only MHC I and IIB respectively, whereas about 10\% of the type IIA and $40 \%$ of the type IIX fibres expressed more than one MHC isoform. Thus IHC detects amounts of myosin isoforms which are not detected by EHC. The MATPase IIX category criterion leaves the possibility that this category contains fibres with myosin type IIA and/or IIB in larger amounts. The criteria of the mATPase categories type I, IIA or IIB preclude assignment to these categories of fibres which also contain other myosin isoforms in larger amounts. Such fibres were classified in one of the mATPase 'Misc' categories. Thus in the EDL the capability of the EHC criteria to select fibres 'pure' in terms of myosin isoform, differs per mATPase category. None of the SM fibres were assigned to the mATPase type I or IIB categories, about 25\% to the type IIA, 60\% to type IIX and 15\% (including most fibres which expressed MHC I) to a 'Misc' category. All SM fibres expressed two or more MHC isoforms, MHC IIB occurring in all fibres and substantial amounts of MHC IIA and/or IIX in most fibres. These findings confirm the hypothesis that such fibres have the capacity to contract fast and have the better fatigue resistance.
\end{abstract}




\subsection{Introduction}

Two small muscles are present in the middle ear of mammals, the stapedius (SM) and the tensor tympani. Contraction of these muscles will result in stiffening of the middle ear bone chain, hence altering the transmission of sound to the cochlea. These middle ear muscles contract upon acoustic and non-acoustic stimuli such as self-phonation, tactile stimuli, and during arousal (Borg et al., 1984; Djupesland, 1975). There are speculations about their function. A widely accepted theory is that the muscles prevent the auditory receptors in the inner ear from injury by intense noise (Fletcher and Riopelle, 1960; Hilding, 1961; Zakrisson, 1975). Acoustic stimulation above about 80 dB results in middle ear muscle contraction which correlates with the intensity of noise (Teig, 1973; Berge et al., 1990). The SM is supposed to be the most relevant in altering the transmission of sound (Berge et al., 1990). It should be able to contract fast and to sustain activity for longer periods if damage due to sudden and longstanding high-level noise exposure is to be prevented. Knowledge of the myosin composition of the SM fibres will contribute to the understanding of the functional properties of the SM.

SM fibres were characterized on the basis of myofibrillar actomyosin adenosine triphosphatase (mATPase) lability after pre-incubation in acid or alkali media and on the basis of activity of enzymes involved in pathways of ATP production. Lyon and Malmgren (1982) reported in the cat 77\% fast oxidative glycolytic fibres (supposed to be equivalent to type IIA fibres) and 23\% smaller fibres, 13\% type I-like fibres and 10\% type II-like fibres, assumed to be comparable to slow oxidative type I and fast glycolytic type IIB (characterization according to Peter et al., 1972). Vegetti et al. (1982) found in horse, sheep, cow, pig, dog, cat and rabbit type I and IIA fibres respectively, in ratios from 20/80\% up to 50/50\%, presumably belonging to slow and fast contracting fatigue resistant motor units. Berge and Wirtz (1989) determined in the rat SM fast (87\%) and slow fibres (5\%), both showing a moderate to high level of succinate dehydrogenase (SDH) and $\alpha$-glycerophosphate dehydrogenase (GPD). Fast-twitch white fibres showing a low level of SDH activity like type IIB fibres were not found in the study by Berge and Wirtz (1989). Burgener and Mayr (1980) combined electron microscopy and mATPase methods; in the guinea pig two thirds of the fibres resembled myotubes and stained for mATPase like IIC fibres, which suggests that they are still in a state of development. One third of the fibres appeared to be more mature, they stained like type I fibres.

It is assumed that the SM mainly contains muscle fibres that are fatigue-resistant and reasonably fast; this suggests, on the basis of findings in skeletal (Larsson and Moss, 1993; Rivero et al., 1998) and masticatory muscles (Bredman et al., 1991), that they contain (combinations of) myosin type IIA, IIX and IIB. The use of different histochemical methods, different animal species and partly superseded classification of contractile components impedes insight in the myosin composition (hybridism) of the SM fibres. Moreover, the presence of myosine type IIX fibres could not be found with the methods used thus far.

Recently developed enzymehistochemical (EHC) and immunohistochemical (IHC) methods allow the determination of the myosin composition of SM fibres more accurately. The EHC fibre characterization 
was extended with a mATPase double pre-incubation procedure which enables the identification of muscle fibres expressing myosin type IIX (Sant'Ana Pereira et al., 1995a). Moreover, this method is said to detect fibres that contain myosin type IIX in combination with either type IIA or IIB.

EHC fibre characterization as was done by Schiaffino et al. (1989) and Gorza (1990), supplemented with the EHC procedure to detect type IIX of Sant'Ana Pereira et al. (1995a), combined with IHC with monoclonal antibodies (mAbs) against myosin heavy chain (MHC) I, IIA, IIX, IIB (Sant'Ana Pereira et al., 1995b; Schiaffino et al., 1989) and cardiac- $\alpha$ (Bredman et al., 1992), has not yet been applied on serial muscle cross-sections, either skeletal or stapedius muscle. Therefore, we investigated the SM of 6 week old rats (which already carries out the adult function) together with a skeletal muscle, the extensor digitorum longus (EDL).

\subsection{Materials and methods}

\subsubsection{Animals, dissection and tissue processing}

Two EDLs were removed in two inbred strain male Brown-Norway rats of 6 weeks and 12 weeks of age respectively. Two SMs were removed completely in two inbred strain male Brown-Norway rats of 6 weeks of age. Care for laboratory animals and all animal procedures were in accordance with the Dutch law on animal care ("Wet op de proefdiervoorziening").

The rat was decapitated and the mandible was removed to show the bulla (tympanic cavity equivalent). The caudal wall of the bulla was opened to approach the pyramidal process (containing the SM muscle belly) and the middle ear bones. The lateral bony wall of the pyramidal process, which protrudes in the tympanic cavity, was broken off. SM fibres did not attach to this bony chip. All muscle fibre bundles of the SM were released from the remaining walls of the pyramidal process by blunt dissection. Then, the SM and the stapes were taken out. The stapes was cut from the SM tendon before stretching the entire SM (mean length $1.2 \mathrm{~mm}$ ) in a drop of Tissue-tek ${ }^{\circledR}$ (0.C.T. compound) poured between two small wooden matches on a slide. Some SM fibres are arranged oblique or more or less perpendicular to the tendon in situ (Berge and Wirtz, 1989). Stretching of the SM reduces longitudinal cutting of these fibres. Then the specimen was frozen in melting isopentane pre-cooled in liquid nitrogen. The muscles were stored in airtight canisters at $-80^{\circ} \mathrm{C}$.

The entire SMs and parts of the EDL were cut to cross-sections $(10 \mu \mathrm{m})$ on a cryostat microtome at $-20^{\circ} \mathrm{C}$. About 100 SM cross-sections were obtained and numbered from the origin to the tendon. Consecutive cross-sections of the EDL parts were numbered also. One SM and one EDL cross-section were mounted on the same slide. Two levels per SM were chosen for fibre type characterization, near SM cross-section numbers 30 and 50. The SM cross-sections of these levels were processed by EHC or IHC and were photographed. A level contains eight consecutive EHC or IHC processed cross-sections. Two levels in two SMs (4x100 fibres) and one level in two EDLs (2×100 fibres) were analyzed. 


\subsection{2 mATPase characterization}

A modification of the procedure of Brooke and Kaiser (1970) was carried out for the acid pre-incubation method (Ogilvie and Feeback, 1990). This method differed from the Brooke and Kaiser method in setting the acid incubation medium at pH 4.35, the incubation taking $45 \mathrm{~s}$ whilst shaking the slides. Type I fibres stained dark, type IIA fibres stained light, type IIX and IIB fibres presented intermediate staining, comparable to the results with the method of Brooke and Kaiser at pH 4.7.

The mATPase alkali lability protocol was adapted from Guth and Samaha (1969). The sections were fixed in buffered 2\% methanol-free paraformaldehyde and stained after pre-incubation at pH 10.4.

The double pre-incubation method according to Sant'Ana Pereira et al. (1995a) was used to determine fibres which contain myosin type IIX.

Fibres were assigned to different mATPase categories on the basis of combinations of staining intensities mentioned in Table 2.1. Myosin ATPase profiles were defined according to Gorza (1990), supplemented with Sant'Ana Pereira's criteria for myosin type IIX (1995a). Fibres which did not meet the criteria of the mATPase categories type I, IIA, IIX or IIB were assigned to type 'Misc' (miscellaneous).

Table 2.1. Combinations of fibre staining on mATPase activity after different pre-incubation on the basis of Gorza (1990) and Sant'Ana Pereira et al. (1995a).

\begin{tabular}{llll}
\hline mATPase category & $\mathrm{pH} 4.35$ & $\mathrm{pH} 10.4$ & Double pre-incubation \\
\hline type I & ++ (dark) & - (light) & - (light) \\
type IIA & - (light) & ++ (dark) & - (light) \\
type IIX & + (intermediate) & ++ (dark) & $+/++$ (intermediate/dark) \\
type IIB & + (intermediate) & + (intermediate) & - (light) \\
type 'Misc' * & mATPase activity profiles other than above & \\
\hline
\end{tabular}

*: Six 'Misc' categories were discerned; only three were found in the EDL.

\subsubsection{MHC characterization}

Each fibre already categorized along the criteria given in Table 2.1 was characterized by immunoreactivity (IR), negative (-) and positive (two intensities, + or ++ ), on a panel of $m A$ bs, respectively specific to rat MHC I (219-1D1), MHC IIA (333-7H1), to both MHC IIA and IIX (332-3D4) (Sant'Ana Pereira et al., 1995b), to MHC IIB (Schiaffino et al., 1989) and to MHC cardiac- $\alpha$ (Bredman et al., 1992). The specificity and sensitivity of these mAbs have been determined on rat, rabbit and/or human skeletal muscles by others (Bredman et al., 1992; Sant'Ana Pereira et al., 1995b; Schiaffino et al., 1989). The indirect unconjugated technique after Moorman et al. (1984) was applied. Sections without primary antibody incubation were used as control. 


\subsubsection{Selection of muscle fibres}

EDL

The complete cross-sections showed an unequal distribution of fibre types as was described by Pullen (1977) and Lexell et al. (1994). We photographed parts of these cross-sections in the middle/ deep part of the muscle. For both EDLs the same region was chosen. These photographs showed about 600 fibres. Selectively located muscle fibre staining, which could be indicative for unequal distribution of muscle fibre types, was not found on these photographs. A hundred adjoining fibres were selected on the cross-section processed for EHC after pre-incubation at $\mathrm{pH} 4.35$. All fibres could be identified on the other cross-sections of the level examined.

\section{SM}

On average 250 fibres were present in each cross-section of the entire muscle. None of the cross-sections processed, by EHC or $\mathrm{HC}$, showed selectively located muscle fibre staining, which could be indicative for unequal distribution of muscle fibre types. An area that only contained cross-sectioned fibres was selected on the photograph of the cross-section processed for EHC after pre-incubation at $\mathrm{pH}$ 4.35 (Fig. 2.1). Then 100 adjacent fibres were numbered in this area. These fibres were identified on the other cross-sections of the given level and numbered correspondingly. A fibre, which could not be found in one of the cross-sections of the level examined was removed from the selection and replaced by an adjoining fibre that showed a staining intensity after pre-incubation at $\mathrm{pH} 4.35$ equal to the excluded fibre. Replacement occurred for about 0-2 fibres per level examined.

Two of the authors independently scored the staining intensity of the selected fibres,-+ or ++ . Scoring was carried out per cross-section. Per level, the score of both examiners of maximally two fibres differed. Assignment of these fibres was established by re-evaluation of the cross-sections in mutual agreement

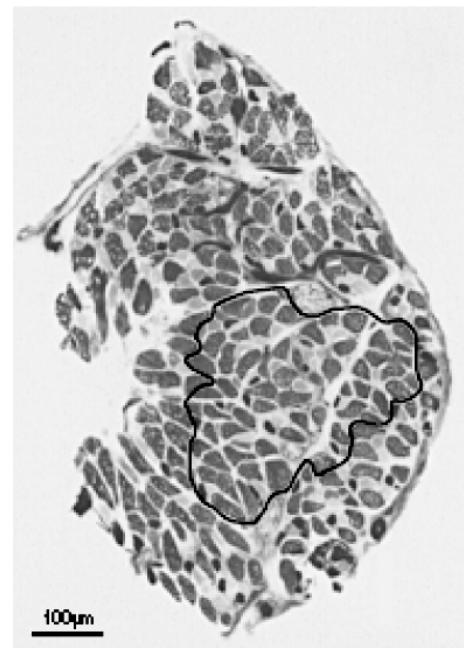

Fig. 2.1. SM cross-section processed by EHC; pre-incubation at $\mathrm{pH}$ 4.35. The fibres in the enclosed area are selected for mATPase and MHC characterization in eight consecutive cross-sections processed by EHC or IHC in this level of the SM (see Fig. 2.3). The SM does not show unequal distribution of muscle fibre types. 
by the two examiners. For illustration, the scoring of five EDL and five SM fibres being,,-+ or ++ is given in Table 2.2 on the basis of the staining qualities of the photographs in Figs. 2.2 and 2.3.

Table 2.2. Staining combinations of five EDL (1-5) and SM (6-10) fibres, from the photographs in Figs. 2.2 and 2.3

\begin{tabular}{|c|c|c|c|c|c|c|c|c|c|}
\hline Fibre & $\mathrm{pH} 4.35$ & $\mathrm{pH} 10.4$ & $\begin{array}{l}\text { Double pre- } \\
\text { incubation }\end{array}$ & $\begin{array}{l}\text { mATPase } \\
\text { category }\end{array}$ & $\alpha$ & I & $\| A$ & $\|A+\| X$ & $\mathrm{IIB}$ \\
\hline 1 & ++ & - & - & 1 & - & ++ & - & - & - \\
\hline 2 & - & ++ & - & $\| \mathrm{A}$ & - & - & ++ & ++ & - \\
\hline 3 & + & + & - & $\| B$ & - & - & - & - & ++ \\
\hline 4 & + & ++ & ++ & $\| X$ & - & - & - & ++ & - \\
\hline 5 & + & ++ & + & $\| X$ & - & - & + & ++ & - \\
\hline 6 & ++ & + & - & 'Misc' & - & ++ & + & - & + \\
\hline 7 & - & ++ & - & $\| A$ & - & - & ++ & ++ & + \\
\hline 8 & + & ++ & ++ & $\| X$ & - & - & - & ++ & + \\
\hline 9 & + & ++ & ++ & $\| X$ & - & - & - & + & ++ \\
\hline 10 & ++ & + & - & 'Misc' & ++ & ++ & - & - & + \\
\hline
\end{tabular}

\subsection{Results}

\subsubsection{EDL}

Almost all fibres (97\% of the 6 weeks EDL and 96\% of the 12 weeks EDL) could be assigned to mATPase categories type I, IIA, IIX or IIB according to the criteria mentioned in Table 2.1. In respectively $55 \%$ and $69 \%$ of the fibres IHC identified only one MHC (groups $A, F, L$ and P). In respectively $74 \%$ and $79 \%$ EHC in combination with IHC identified only one myosin (groups $A, F, L, P$ and $B, C, D$ ) (Table 2.3A).

All fibres in the mATPase categories type I and IIB only expressed the corresponding MHC. Four fibres in the mATPase category type IIA expressed MHC IIB besides the corresponding MHC (group E). Almost one third up to half of the fibres in the mATPase category type IIX expressed MHC IIB (group G, H and I) or MHC IIA (group J and K) besides showing IR to the mAb to both MHC IIA and IIX. The fibres in groups J and $K$ presented an IR pattern similar to groups $B, C$ and $D$, but differed with respect to their mATPase activity profile (mATPase type IIX versus mATPase type IIA) (Table 2.3B).

The fibres (3\%, respectively 4\%) assigned to different mATPase type 'Misc' categories were hybrid fibres (except for one fibre in group $\mathrm{P}$, which only expressed MHC IIB). Group M and $\mathrm{Q}$ co-expressed MHC I and IIA, a type of co-expression that was not found in other EDL fibres. Groups N and 0 co-expressed MHC IIB and MHC IIA and/or IIX, a type of co-expression, which was also found in the groups $\mathrm{G}, \mathrm{H}$ and I. However, the groups $\mathrm{G}, \mathrm{H}$ and I had a mATPase activity profile type IIX. 

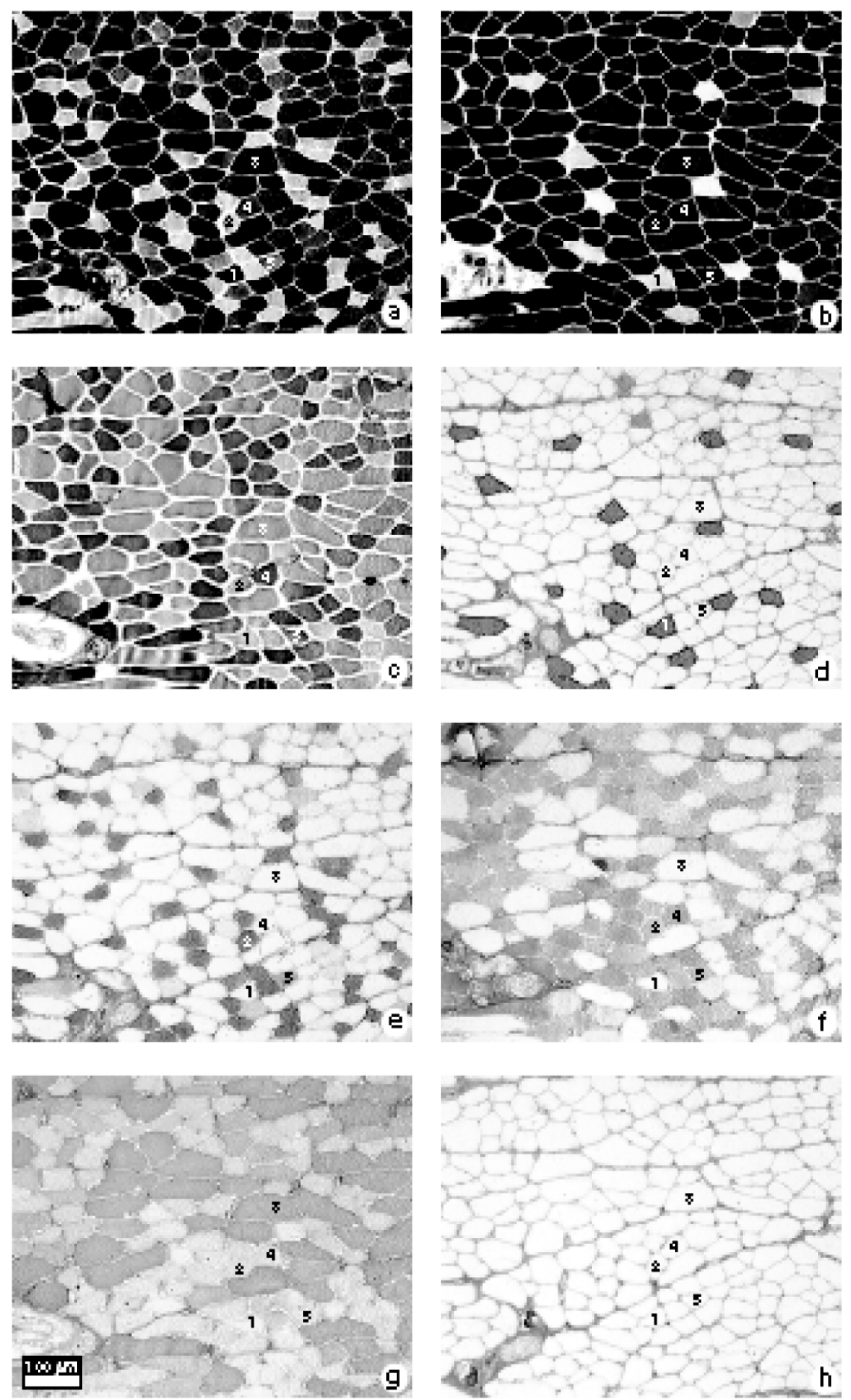

Fig. 2.2. EDL muscle fibre mATPase characterization (a: $\mathrm{pH} 4.35, \mathrm{~b}: \mathrm{pH} 10.4$, c: double pre-incubation) and MHC characterization (d:I, e: IIA, f: both IIA and IIX, g: IIB, $h$ : cardiac- $\alpha$ ). Staining intensities of the fibres numbered 1-5 are given in Table 2.2. Note: Only 2 fibres in the muscle spindle are positive for cardiac- $\alpha$ 

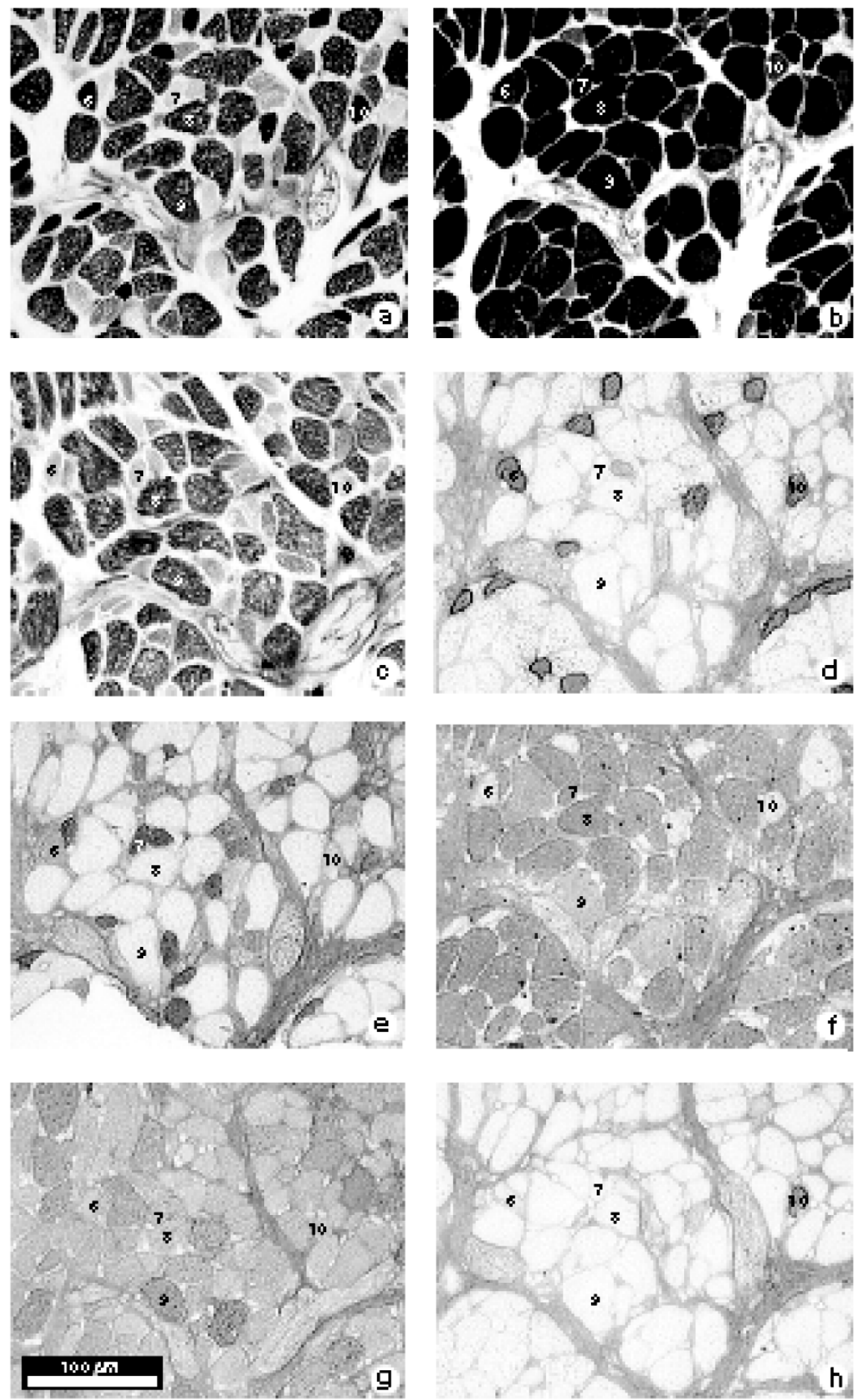

Fig. 2.3. SM fibre mATPase characterization (a: $\mathrm{pH} 4.35, \mathrm{~b}: \mathrm{pH}$ 10.4, c: double pre-incubation) and MHC characterization (d: I, e: IIA, f: both IIA and IIX, g: IIB, h: cardiac- $\alpha$ ). Staining intensities of the fibres numbered 6-10 are given in Table 2.2. 
Table 2.3. EDL fibres assigned to mATPase categories type I, IIA, IIX and IIB (A) and 'Misc' (B).

\begin{tabular}{|c|c|c|c|c|c|c|c|c|}
\hline \multirow[t]{2}{*}{ mATPase category } & \multirow[t]{2}{*}{ 6/12 weeks } & \multirow[t]{2}{*}{ IHC group } & \multicolumn{2}{|c|}{$\mathrm{mAb} I \mathrm{R}$} & \multirow[b]{2}{*}{$\| A$} & \multirow[b]{2}{*}{$\| \mathrm{B}$} & \multirow[b]{2}{*}{ IIAX } & \multirow[t]{2}{*}{$6 / 12$ weeks } \\
\hline & & & $\alpha$ & I & & & & \\
\hline \multicolumn{9}{|l|}{$A^{a}$} \\
\hline type I & $8 / 7$ & $A$ & - & ++ & - & - & - & $8 / 7$ \\
\hline \multirow[t]{4}{*}{ type IIA } & $21 / 12$ & $B$ & - & - & ++ & - & ++ & $17 / 9$ \\
\hline & & C & - & - & + & - & + & $1 / 0$ \\
\hline & & $\mathrm{D}$ & - & - & ++ & - & + & $1 / 1$ \\
\hline & & $E$ & - & - & ++ & + & ++ & $2 / 2$ \\
\hline \multirow[t]{6}{*}{ type IIX } & $40 / 51$ & $\mathrm{~F}$ & - & - & - & - & ++ & $19 / 35$ \\
\hline & & G & & - & - & ++ & + & $1 / 6$ \\
\hline & & $\mathrm{H}$ & - & - & - & + & ++ & $11 / 8$ \\
\hline & & । & - & - & - & + & + & $3 / 1$ \\
\hline & & J & - & - & ++ & - & ++ & $1 / 1$ \\
\hline & & K & - & - & + & - & ++ & $5 / 0$ \\
\hline type IIB & $28 / 26$ & $L$ & - & - & - & ++ & - & $28 / 26$ \\
\hline \multicolumn{9}{|l|}{$\mathrm{B}^{b}$} \\
\hline type 'Misc' (1) & $1 / 0$ & $M$ & - & ++ & + & - & - & $1 / 0$ \\
\hline \multirow[t]{3}{*}{ type 'Misc' (2) } & $0 / 4$ & $\mathrm{~N}$ & - & - & - & + & + & $0 / 2$ \\
\hline & & 0 & - & - & - & + & + & $0 / 1$ \\
\hline & & $P$ & - & - & - & - & - & $0 / 1$ \\
\hline type 'Misc' (3) & $2 / 0$ & 0 & - & + & ++ & - & - & $2 / 0$ \\
\hline
\end{tabular}

a The EDL fibres are subdivided to MHC combination groups $A-L$ on the basis of IR to five mAbs. Numbers in $A$ together with B represent 100 fibres in each EDL; one level muscle 6 weeks and one level muscle 12 weeks of age. 'The mATPase activity profiles of the categories type 'Misc' (staining after pre-incubation pH 4.35, 10.4 or Double pre-incubation) are (1),,,$+++-(2)+,++,-$ and $(3)-,+,-$. The fibres are subdivided to MHC combination groups $\mathrm{M}-0$ on the basis of IR to five mAbs.

\subsubsection{SM}

The number of SM fibres that could be assigned to mATPase categories type I, IIA, IIX or IIB according to the criteria mentioned in Table 2.1 was smaller than that of the EDL (83.5\% versus 97/96\%). None of the fibres could be assigned to the mATPase categories type I or type IIB. Besides, three additional mATPase type 'Misc' categories were found in the SM (6 versus 3 in the EDL). All fibres showed IR (+ or ++) to two or more mAbs, one constantly being the mAb to MHC IIB (Table 2.4A).

All fibres in the mATPase category type IIA showed IR (mainly ++) to the mAb to MHC IIA and to the mAb to both MHC IIA and IIX. A part (groups $d$ and e) also expressed MHC I and cardiac- $\alpha$ (group e) which was 
Table 2.4 SM fibres assigned to mATPase categories type I, type IIA, type IIX and type IIB (A) and type 'Misc' (B)

\begin{tabular}{|c|c|c|c|c|c|c|c|c|}
\hline mATPase category & Mean \% & IHC group & & & & & & Mean $\%$ \\
\hline & & & $\alpha$ & I & $\| A$ & $\| \mathrm{B}$ & $\| A X$ & \\
\hline \multicolumn{9}{|l|}{$A^{a}$} \\
\hline Type I & 0.00 & & & & & & & 0.00 \\
\hline \multirow[t]{5}{*}{ Type IIA } & 23.50 & $a=E$ & - & - & ++ & + & ++ & 14.25 \\
\hline & & $b$ & - & - & + & ++ & ++ & 1.00 \\
\hline & & c & - & - & ++ & ++ & ++ & 2.25 \\
\hline & & $d$ & - & + & ++ & + & ++ & 5.50 \\
\hline & & e & + & + & ++ & + & ++ & 0.50 \\
\hline \multirow[t]{4}{*}{ Type IIX } & 60.00 & $f$ & - & - & - & ++ & ++ & 5.75 \\
\hline & & $g=G$ & - & - & - & ++ & + & 3.00 \\
\hline & & $h=H$ & - & - & - & + & ++ & 43.00 \\
\hline & & i & - & - & + & + & ++ & 7.75 \\
\hline Type IIB & 0.00 & & & & & & & 0.00 \\
\hline \multicolumn{9}{|l|}{$\mathrm{B}^{\mathrm{b}}$} \\
\hline \multirow[t]{4}{*}{ Type 'Misc' (1) } & 9.75 & j & + & ++ & + & + & - & 4.00 \\
\hline & & k & - & ++ & + & ++ & - & 0.50 \\
\hline & & 1 & - & ++ & + & + & - & 3.25 \\
\hline & & $\mathrm{m}$ & - & ++ & - & + & - & 2.00 \\
\hline \multirow[t]{2}{*}{ Type 'Misc' (4) } & 2.75 & $n=1$ & - & ++ & + & + & - & 2.50 \\
\hline & & $0=j$ & + & ++ & + & + & - & 0.25 \\
\hline Type 'Misc' (5) & 0.25 & $p$ & + & ++ & - & + & ++ & 0.25 \\
\hline \multirow[t]{3}{*}{ Type 'Misc' (2) } & 1.75 & $q$ & - & ++ & ++ & + & + & 1.25 \\
\hline & & r & + & ++ & ++ & + & + & 0.25 \\
\hline & & $\mathrm{s}$ & - & + & + & + & + & 0.25 \\
\hline Type 'Misc' (3) & 0.25 & $t=c$ & - & - & ++ & ++ & ++ & 0.25 \\
\hline \multirow[t]{2}{*}{ Type 'Misc' (6) } & 1.75 & $u=i$ & - & - & + & + & ++ & 0.25 \\
\hline & & $\mathrm{V}=\mathrm{a}$ & - & - & ++ & + & ++ & 1.50 \\
\hline
\end{tabular}

a The SM fibres are subdivided to MHC combination groups a-i on the basis of IR to five mAbs. (Mean percentages in A together with B represent 4x 100 fibres; two levels muscle 1 and two levels muscle 2, both 6 weeks of age).

${ }^{b}$ The mATPase activity profiles of categories type 'Misc' 1-3 are described in footnote to Table 2.3. The profiles of the other mATPase categories type 'Misc' (pH 4.35, 10.4 or Double pre-incubation) are (4),,,$++++-(5)++,+,+$ and (6) - , ,+++ . The fibres are subdivided to MHC combination groups $j-v$ on the basis of IR to five mAbs. 
not found in the mATPase category type IIA in the EDL muscle. The fibres in the mATPase category type IIX showed IR to the mAb to both MHC IIA and IIX. A part (group i) also expressed MHC IIA, which was also found in the mATPase category type IIX in the EDL (groups J and K). Groups in mATPase type IIA and type IIX categories with an identical IR combination (as described for the EDL) were not found (Table 2.4B). The largest number of the SM fibres that showed strong (++) IR to the mAb to MHC I and (++) mATPase activity after pre-incubation at pH 4.35 were assigned to the mATPase category type 'Misc' (1), (4) or (5). Moreover, the fibres in these categories which showed (+) IR to the mAb to MHC IIA together with (++) IR to the $\mathrm{mAb}$ to $\mathrm{MHCI}$ (groups $\mathrm{j}, \mathrm{k}, \mathrm{l}, \mathrm{n}$ and o) were found to be (-) to the mAb to both MHC IIA and IIX. Table 2.4 shows that fibres which demonstrated strong (++) IR to the mAb to MHC IIB were assigned to many mATPase categories; type IIA (groups $b$ and c), type IIX (groups $f$ and $g$ ) and the type 'Misc' (1) and (3) (groups $k$ and $t$ ).

The groups $t$ and $v$ showed a combination of IR to mAbs, which was also found in groups of the type IIA mATPase category (groups $c$ and a respectively). The IR combination of the groups $u$ and $i$ also were the same. The last group belonged to the mATPase type IIX category. Amongst the mATPase categories type 'Misc' some groups also shared the same IHC IR combination ( $n=1,0=j)$.

\subsection{Discussion}

The fibre characterization method used in this study differs from previous methods. Therefore, the EDL results of the 6 and 12 weeks old rat are compared to EDL data in the literature. It will be argued that the present method offers advantages in interpreting the results of the SM, a muscle that shows a more complicated fibre composition than the EDL.

\subsubsection{EDL characterization compared to previous EDL results}

Lodder et al. (1993) showed the following fibre composition in the EDL muscle of rats at 60 days of age on the basis of mATPase histochemistry: 5\% type I, 21\% type IIA, 35\% type IIX and 38\% type IIB, furthermore 1\% type IIC fibres were mentioned. Our EDLs (aged 6/12 weeks) showed a comparable mATPase type composition: 8/7\% type I, 21/12\% type IIA, 40/51\% type IIX and 28/26\% type IIB. EHC found 3\% of the EDL fibres containing more than one myosin. They were assigned to mATPase type 'Misc' categories. The IIC fibres appeared to have the same mATPase profile as our 'Misc' (1) category fibres. All EDL fibres in our mATPase categories type I and type IIB were 'pure' (i.e. IHC showed one MHC type) in contrast with many fibres in the mATPase category type IIX being hybrid.

The combined application of EHC and IHC is indispensable to determine the myosin composition as was stated previously (Sant'Ana Pereira et al., 1995b). Besides, our EDL results suggest that this combination gives an impression of the amounts of myosin present in fibres that contain more than one myosin. Firstly, IHC detects (small) amounts of myosin that were not detected by EHC; it was found in some fibres in the mATPase category type IIA that small amounts of myosin type IIB (MHC IIB detected by IHC) did 
not influence fibre's EHC profile. Secondly, EHC only demonstrates larger amounts of myosin; fibres that contained several myosins in larger amounts were assigned to a mATPase category type 'Misc', because the larger amounts of myosin type I and IIA or IIB in these fibres caused staining after different preincubations. Thirdly, by means of IHC we found larger numbers of hybrid fibres in the mATPase category type IIX (co-expression of MHC IIA and/or IIB) than in the mATPase categories type I, IIA and IIB. EHC cannot detect whether fibres which contain myosin type IIX and IIA and/or IIB show positive staining after pre-incubation at pH 4.35 or $\mathrm{pH} 10.4$ on the basis of their content of myosin type IIX, IIA or IIB. We conclude that the EHC profile of the mATPase type IIX category (Table 2.1) allows assigning of fibres which co-express larger amounts of MHC IIA and/or IIB. This profile impedes the assignment of hybrid fibres to a mATPase type 'Misc' category. The mATPase type I, IIA and IIB profiles select 'pure' fibres more specifically. Finally, our EDL study confirms the statement of Sant'Ana Pereira et al. (1995a) that IHC cannot be conclusive on the myosin composition in fibres showing (+) or (++) IR to the mAb to MHC IIA combined with (+) or (++) IR to the mAb to both MHC IIA and IIX. EHC gives the character (mATPase type IIA or type IIX). EHC in combination with IHC suggests that these fibres contain more than one myosin in case they were assigned to the mATPase type IIX category. We were unable to quantify the amount of myosin type IIX after applying the double pre-incubation EHC method of Sant'Ana Pereira et al. (1995a), because of the lack of a staining gradient in the EDL mATPase type IIX fibres (or SM, which all are a grade lighter than in the EDL).

\subsubsection{SM fibre composition}

All SM fibres displayed MHC hybridism. This may indicate incomplete differentiation (Whalen et al., 1981; Butler-Browne et al., 1988; Staron and Pette, 1993). A histochemical, light and electron microscopic study on the guinea pig SM favours the hypothesis that SM fibres are "more or less undifferentiated and show the persistence of developmental properties" (Burgener and Mayr, 1980). In contrast to these findings in the guinea pig, electron microscopy on SM of the adult rat failed to show features, which point to incomplete differentiation (Berge and Wirtz, 1989). Moreover, polyneural innervation, described in developing muscle fibres or in some fibres in mammalian extraocular muscles (Schmalbruch, 1985) was not found in the stapedius muscle (Berge and Wal, 1990). SM fibres coexpressing MHC cardiac- $\alpha$ (5.5\%) may point to incomplete differentiation, as was described for the developing semitendinous muscle of the piglet (Lefaucheur et al., 1995), but it also may reflect the branchial origin of the SM. This MHC was found also in the masseter muscle of the adult rat, a finding that was associated with its branchial origin (Bredman et al., 1992). These data do not support that SM fibre hybridism is a sign of retained development. A large portion of fibres being hybrid was also shown in adult skeletal muscles (Larsson and Moss, 1993). We suppose that the MHC hybridism of SM fibres reflects functional demands of a muscle in a full-grown middle ear in the 6 weeks old rat. The tuning of sound transmission by the SM requires the expression of all MHCs. Firstly, hybrid muscle fibres may permit a finer gradation of contraction speeds per fibre (Bredman et al., 1991). Secondly, fibres co-expressing two MHC isoforms generally showed intermediate SDH and GPD values lying between 
their respective pure MHC fibre types (Rivero et al., 1998). This suggests that such fibres have effective aerobic as well as anaerobic metabolic capabilities to generate ATP. Thus, muscle fibre properties such as are ascribed to hybrid fibres enable the hybrid SM fibres (many fibres co-express MHC IIX, IIA and IIB) to have the disposal of a favourable contraction speed and fatigue resistance, to contract fast and to sustain activity for longer periods.

Our study showed a different SM fibre composition than was described previously. Although about 45\% of the type II fibres expressed MHC IIA to some extent, the MATPase type IIA fibres were not the prevalent type as was suggested by Lyon and Malmgren (1982), Vegetti et al. (1982) or Berge and Wirtz (1989). Myosin ATPase type IIX fibres dominated. They nearly all showed (++) IR against the mAb to both MHC IIA and IIX. However, a part of these fibres showed co-expression of MHC IIB and MHC IIA. The amounts of myosin type IIA or IIB in these fibres could have been substantial, because as argued before, fibres with larger amounts of myosin type IIA or IIB can be assigned to the mATPase type IIX category. Only $23.5 \%$ of the fibres was assigned to the mATPase type IIA category, IHC showed that about one quarter of these fibres contained MHCI besides MHC IIA. These fibres were not assigned to a mATPase type 'Misc' category, which suggests that they contained myosin type I in small amounts. The SM did not contain 'pure' type I fibres. Fibres with (++) IR to the mAb to MHC I (supposedly containing the largest amount of myosin type I) were assigned to several mATPase type 'Misc' categories, because of (+) staining after pre-incubation at pH 10.4 indicating substantial amounts of type II myosin isoforms in these fibres. The SM did not contain 'pure' type IIB fibres either. All fibres expressed MHC IIB, however we suppose that only a few fibres contained myosin type IIB in substantial amounts. Such fibres are supposed to show $(++)$ IR to the $\mathrm{mAb}$ to MHC IIB and positive mATPase activity staining after pre-incubation at pH 4.35 . They were only found in the mATPase type IIX and type 'Misc' (1) categories.

Our results differ from previous SM descriptions and a muscle with all fibres containing more than one myosin has not been reported before. Only van den Berge and Wirtz (1989) stated that it is most likely that in the SM of the rat different, fully differentiated heavy chain myosin types do occur in one fibre. This was suggested however on the basis of the finding that all fibres stained with equal (weak) intensity on two of their mAbs used.

Although inaccurate application of the method is unlikely in view of the results of the co-processed EDLs on the same slides, this hybridism induced supplementary investigations about the accuracy of our fibre characterizations.

Firstly, we processed sections that were pre-incubated at pH 11.0 and 11.2; all fibres were unstained. This suggests that (+) staining of the SM fibres after pre-incubation at $\mathrm{pH} 10.4$ was indeed caused by a type II mATPase activity. A general MHC IIB expression was found in SMs of the 12 weeks old rat. Non-specific binding could have caused SM fibres being positive for the MAb to MHC IIB. Therefore, a SDS-PAGE analysis of the 6 and 10 weeks old rat SM was carried out (unpublished data). This showed the presence of MHC IIB. Moreover, about 10\% of the SM fibres stained (++) for the mAb to MHC IIB in contrast to (+) staining of the other fibres. The ++ staining intensity corresponded to the (++) staining in the EDL fibres. These findings suggest that the $\mathrm{mAb}$ to MHC IIB indeed demonstrated this MHC. 
Secondly, Staron and Pette (1987) noted that a skeletal muscle fibre might contain different myosins at different cross-sectional levels. Therefore, we verified whether SM fibres showed a constant EHC staining over a series of 10 consecutive cross-sections (series after pre-incubations at pH 4.35, 4.40, 10.4 and the double pre-incubation technique respectively). This could be confirmed. Finally, the mAb to both MHC IIA and IIX seems to be less sensitive than the MAb to MHC IIA. This can be concluded, because some SM fibres with a (+) IR to the mAb to MHC IIA showed a (-) IR to the MAb to both MHC IIA and IIX. The reason why this concerned fibres that show a (++) IR to the $\mathrm{mAb}$ to $\mathrm{MHC}$ I remains unclear. This phenomenon was found in only a few EDL fibres and therefore supposedly it was not reported by others before.

These methodological remarks support, that our conclusion (all SM fibres contained more than one myosin) is not caused by misinterpretation of the staining gradient, by muscle fibres having a different myosin composition at different levels or by non-specificity of the mAbs.

\subsection{Conclusions}

The application of the EHC and IHC combination on the EDL shows the functional character of a hybrid fibre in terms of myosin dominance, which is necessary to understand the functional character of the SM (all fibres being hybrids). At 6 weeks all SM fibres contained fast type II myosins. Even the fibres which contained myosin type I in larger amounts contained myosin type II isoforms in substantial amounts or cardiac- $\alpha$ MHC, which is considered to be much faster than myosin type I (VanBuren et al., 1995). We supposed that the MHC hybridism in the rat SM is a quality of a full-grown muscle adapted to its function rather than a reflection of a developmental stage. The predominance of myosin types II in the hybrid fibres gives the SM the capability to react fast (high Vmax). Myosin types II which are associated most closely with fatigue resistance, were found in the majority of the fibres. This means that the SM can sustain activity for longer periods. Therefore, we conclude that the SM of the 6 week old rat is well equipped to protect auditory receptors from injury.

Acknowledgements; this study was supported by the Heinsius-Houbolt Foundation. We kindly thank Prof. Stefano Schiaffino for the possibility of using the mAb against MHC IIB in this study. 


\section{References}

- $\quad$ Berge, H. van den, Wirtz, P., 1989. Detailed morphology of the stapedius muscle of the rat. J. Anat. 166, 157-169.

- Berge, H. van den, Kingma, H., Kluge, C., Marres, E.H.M.A., 1990. Electrophysiological aspects of the middle ear muscle reflex in the rat: Latency, rise time and effect on sound transmission. Hear. Res. 48, 209-219.

- $\quad$ Berge, H. van den, Wal, J.C. van der, 1990.The innervation of the middle ear muscles of the rat. J. Anat. 170, 99-109.

- Borg, E., Counter, S.A., Rosler, G., 1984. Theories about middle ear muscle function. In: Silman, S. (Ed.), The Acoustic Reflex; Basic Principles and Clinical Applications. Academic Press, Orlando Fl, pp. 50-87.

- Bredman, J.J., Wessels, A., Weijs, W.A., Korfage, J.A., Soffers, C.A., Moorman, A.F., 1991. Demonstration of cardiac-specific myosin heavy chain in masticatory muscles of human and rabbit. Histochem. J. 23, 160-170.

- Bredman, J.J., Weijs, W.A., Moorman, A.F.M., 1992. Presence of cardiac $\alpha$-myosin correlates with histochemical myosin Ca ${ }^{2+}$ ATPase activity in the rabbit masseter muscle. Histochem. J. 24, 260-265.

- $\quad$ Brooke, M.H., Kaiser, K.K., 1970. Muscle fibre types how many and what kind? Arch. Neurol. 23, 369-379.

- Burgener, J., Mayr, R., 1980. Guinea pig stapedius muscle. A histochemical, light and electron microscopic study. Anat. Embryol. $161,65-81$.

- $\quad$ Butler-Browne, G.S., Eriksson, P.O., Laurent, C., Thornell, L.E., 1988. Adult human masseter muscle fibres express myosin isozymes characteristics of development. Muscle Nerve 11, 610-620.

- Djupesland, G., 1975. Advanced reflex considerations. In: Jerger, J.J. (Ed.), Handbook of Clinical Impedance Audiometry. American Electromedics Cooperation, Dobbs Ferry, New York, pp. 85-125.

- $\quad$ Fletcher, J.L., Riopelle, A.J., 1960. The protective effect of the acoustic reflex for impulsive noise. J. Acoust. Soc. Am. 32, 401404.

- Gorza, L., 1990. Identification of a novel type 2 fibre population in mammalian skeletal muscle by combined use of histochemical myosin ATPase and anti-myosin monoclonal antibodies. J. Histochem. Cytochem. 38, 257-265.

- Guth, L. and Samaha, F.J., 1969. Procedure for the histochemical demonstration of actomyosin ATPase. Exp. Neurol. 28, 365367.

- Hilding, D.A., 1961. The protective value of the stapedius reflex: An experimental study. Trans. Am. Acad. Ophtalmol. Otolaryngol. 65, 297-307.

- Larsson, L., Moss, R.L., 1993. Maximum velocity of shortening in relation to myosin isoform composition in single fibres from human skeletal muscles. J. Physiol. 472, 595-614.

- Lefaucheur, L., Edom, F., Ecolan, P., Butler-Browne, G.S., 1995. Pattern of muscle fibre type formation in the pig. Dev. Dyn. 203, 27-41.

- Lexell, J., Jarvis, J.C., Currie, J., Downham, D.Y., Salmons, S., 1994. Fibre type composition of rabbit tibialis anterior and extensor digitorum longus muscles. J. Anat. 185, 95-101.

- Lodder, M.A., De Haan, A., Lind, A., Sargeant, A.J., 1993. Changes in morphological and functional characteristics of male rat EDL muscle during growth. J. Muscle Res. Cell. Motil. 14, 47-53.

- Lyon, M.J., Malmgren, L.T., 1982. A histochemical characterisation of muscle fibre types in the middle ear muscles of the cat. Acta Otolaryngol. 94, 99-109.

- Moorman, A.F.M., Boer, P.A.J. de, Linders, M.Th., Charles, R., 1984. The histone H5 variant in Xenopus laevis. Cell. Differ. 14, 113123. 
- Ogilvie, R.W., Feeback, D.L., 1990. A metachromatic dye-ATPase method for the simultaneous identification of skeletal muscle fibre types I, IIA, IIB and IIC. Stain Technol. 65, 231-241.

- $\quad$ Peter, P.B., Barnard, R.J., Edgerton, V.R., 1972. Metabolic profiles of three fibre types of skeletal muscle in guinea pigs and rabbits. Biochemistry 11, 2627-2633.

- Pullen, A.H., 1977. The distribution and relative sizes of fibre types in the extensor digitorum longus and soleus muscle of the adult rat. J. Anat. 123, 467-486.

- $\quad$ Rivero, J.L., Talmadge, R.J., Edgerton, V.R., 1998. Fibre size and metabolic properties of myosin heavy chain-based fibre types in rat skeletal muscle. J. Muscle Res. Cell Motil. 19, 733-742.

- Sant'Ana Pereira, J.A.A., Haan, de A., Wessels, A., Moorman, A.F.M., Sargeant, A.J., 1995a. The mATPase histochemical profile of rat type IIX fibres: Correlation with MyHC immunolabelling. Histochem. J. 27, 715-722.

- Sant'Ana Pereira, J.A.A., Wessels, A., Nijtmans, L., Moorman, A.F.M., Sargeant, A.J., 1995b. New method for the accurate characterisation of single human skeletal muscle fibres demonstrates a relation between mATPase and MyHC expression in pure and hybrid fibre types. J. Muscle Res. Cell Motility 16, 21-34.

- Schiaffino, S., Gorza, L., Sartore, S., Saggin, L., Ausini, S, Vianello, M., Gundersen, K., Lomo, T., 1989. Three myosin heavy chain isoforms in type 2 skeletal muscle fibres. J. Muscle Res. Cell Motility 10, 197-205.

- Schmalbruch, H., 1985. Non-skeletal muscles. In: Schmalbruch, H. (Ed.), Skeletal Muscle (Handbook of Microscopic Anatomy). Volume II/6, Springer-Verlag, Berlin, pp. 217-238.

- Staron, R.C., Pette, D., 1987. Nonuniform myosin expression along single fibres of chronically stimulated and contralateral rabbit tibialis anterior muscles. Pflügers Arch. 409, 67-73.

- Staron, R.C., Pette, D., 1993. The continuum of pure and hybrid myosin heavy chain based fibre types in rat skeletal muscles. Histochemistry 100, 149-153.

- Teig. E., 1973. Differential effect of graded contraction of middle ear muscles on the sound transmission of the ear. Acta Physiol. Scand. 88, 382-391.

- VanBuren, P., Harris, D.E., Alpert, N.R., Warshaw, D.M., 1995. Cardiac V1 and V3 myosins differ in their hydrolytic and mechanical activities in vivo. Cir. Res. 77, 439-444.

- Vegetti, A., Mascarello, F., Carpene, E., 1982. A comparative histochemical study of fibre types in middle ear muscles. J. Anat. $135,333-352$.

- Whalen, R.G., Sell, S.M., Butler-Browne, G.S., 1981. Three myosin heavy-chain isozymes appear sequentially in rat muscle development. Nature 292, 805-809.

- Zakrisson, J.E., 1975. The role of the stapedius reflex in post-stimulatory auditory fatigue. Acta Otolaryngol. 79, 1-10. 
- 


\section{Chapter 3}

\section{Stapedius muscle fiber characterization during postnatal development in the rat}

Patrick F.M. Dammeijer ${ }^{\text {}, ~ P a u l ~ v a n ~ D i j k k ~}{ }^{2}$, Johannes J. Manni ${ }^{1}$, Henk van Mameren ${ }^{2}$

Department of Otorhinolaryngology and Head and Neck Surgery, University Hospital Maastricht, P.0. Box 5800, 6202 AZ Maastricht, The Netherlands

2 Department of Anatomy/Embryology, University of Maastricht, P.O. Box 616, 6200 MD Maastricht, The Netherlands

Published in Hearing Research 2006; 219(1-2), 48-55. 


\begin{abstract}
The stapedius muscle (SM) is reported to prevent cochlear damage by noise. Functional demands are then the ability of fast contraction with long endurance. At the end of the third postnatal week, the middle ear of the rat is completely pneumatized and according to electrophysiological data, the auditory function starts to match the adult.

We investigated the developmental changes in myosin composition of SM fibres using consecutive complete SM cross-sections (taken from rats on post natal day (PND) 7, 14, 16, 21, 28, 42 and 84)) which were processed by enzymehistochemistry to determine acid/alkali lability of myofibrillar adenosine triphosphatase (mATPase) and by immunohistochemistry using myosin heavy chain (MHC) antibodies (mAb). Fibres were assigned to mATPase type I, IIA, IIB, IIX or 'Miscellaneous' categories. Per mATPase category, the fibres were attributed to groups with specific MHC isoform compositions. Neonatal MHC expression could not be documented with the mAb used. However, embryonal (Emb) MHC was expressed at PND 7, very little at PND 14; at later PND fibres did not show Emb MHC. In general, the mATPase-based classification did not show large alterations after PND 21. Expression of MHC IIB, which was present in almost 50\% of the fibres at PND 7 and 14, diminished to 3\% at PND 84. A decrease in number of fibres expressing more than one MHC isoform was found. These results show that the SM is a precociously developing muscle compared to limb muscles and even to the diaphragm. Moreover, it is shown that the expression of the adult MHC isoform phenotype coincides with the onset of auditory function in the third postnatal week.
\end{abstract}




\subsection{Introduction}

The stapedius and tensor tympani in the middle ear of mammals are the smallest striated muscles in the body. Contraction of these muscles influences transmission of sound through the middle ear. In this respect, the stapedius muscle (SM) is supposed to be the most relevant in man (Møller, 1965) as well as in the rat (Berge et al., 1990; Pilz et al., 1997). The SM shows activity after a variety of stimuli. Acoustic as well as nonacoustic stimuli (Ono et al., 1980) and self-vocalization causes the SM to contract (Borg and Zakrisson, 1975).

Several functions have been attributed to the SM. For instance, it is supposed that the contraction of the SM protects the subject from noise induced hearing loss (NIHL) (Fletcher and Riopelle, 1960; Borg et al., 1984; Zakrisson, 1975). Contraction of the SM would decrease the masking of high-frequency signals by low-frequency noise (i.e., the upward spread of masking) (Borg et al., 1984; Pang and Guinan, 1997) and therefore should improve perception of speech in a noisy setting (Wormald et al., 1995).

To gain insight into the proposed function of the SM much research has been conducted to the physiological and morphological properties of the muscle fibres of the SM in various animal species. (For references, see Dammeijer et al., 2000). Adult mammalian skeletal muscle is differentiated into distinct fibre types, each of which possesses a unique combination of functional, biochemical, and metabolic properties (Schiaffino et al., 1990). A characteristic feature defining this spectrum of fibre types is the type of myosin heavy chain (MHC) isoform that is expressed. In adult rodents, four adult MHC isoforms have been identified in limb muscles, and have been designated as the types I (or cardiac-B), IIA, IIX, and IIB MHC isoforms in increasing order of their ATPase activity and hence fibre-shortening properties. Muscles used extensively for antigravity function and postural support chiefly contain fibres expressing either the type I or type IIA MHC, whereas muscles chiefly recruited for high-intensity power output for brief periods of time express primarily the type IIX and type IIB MHC isoforms (Hoh, 1992; Schiaffino et al., 1990). Other MHC isoforms have been described; super fast IIM MHC and laryngeal or extraocular MHC which are expressed in a few specialized muscles (Wieczorek et al., 1985; Perie et al., 2000). The cardiac- $\alpha$ MHC isoform is expressed in the masseter muscle of the adult rat (Bredman et al., 1992) and the SM of the rat (Dammeijer et al., 2000), a finding that was associated to the pharyngeal arch origin of these muscles. In addition, two developmental MHC isoforms have been identified in striated muscles during the fetal/neonatal period, and they have been designated as embryonic (Emb) MHC and neonatal (Neo) MHC.

During postnatal skeletal muscle development the pattern of $\mathrm{MHC}$ isoform expression features two successive phases. The first is characterized by a down-regulation and elimination of Emb and Neo MHCs. The second phase is characterized by an up-regulation and stabilization of the adult MHC phenotype (Agbulut et al. 2003). Studies on postnatal development of muscles in rats showed programs of myosin expression for each muscle specifically. The sequential transition of myosin isoforms during postnatal development is controlled by multiple factors such as functional demand, innervation, calcium influx and hormonal signals (Schiaffino and Reggiani, 1996; Pette and Staron, 2000). 
The role of functional demands such as locomotion and feeding on muscle development are described in rats for, respectively, limb muscles and the masseter in the first postnatal month (Butler-Browne and Whalen, 1984; Usami et al. 2003). In rats, diaphragm and intercostals at birth already contain adult MHC isoforms and were considered the most precocious of all those examined (d'Albis et al., 1989). This suggests that the speed of the transition towards adult isomyosins reflects the importance of mature functioning of these muscles soon after birth. We hypothesize that in the rat SM histochemical and immunohistochemical adaptations occur in relation to changes of the transmission of sound through the middle ear. Muscle fibre type changes in the SM in relation to the maturation of the auditory function have not been investigated thus far.

In the first postnatal week the tympanic bulla in rats, the equivalent of the middle ear, contains a gelatinous mass, in which the auditory ossicles are embedded and the SM and tensor tympani come into being. At the beginning of the third postnatal week, the tympanic bulla is completely pneumatized and shows essentially the same three-dimensional organization as in the adult rat. In the rat, the onset of auditory function as monitored by recording Auditory Brainstem evoked Responses (ABR) was reported to begin on postnatal day (PND) 12-14 and reaches near adult thresholds at about PND 22 (Geal-Dor et al. 1993). At this time a functional maturation of the SM seems desirable in order to execute the tasks necessary.

In this study, we determined the changes of MHC isoform expression in the SM from birth to maturity in relation to the maturation of the auditory system. The MHC isoform expressions were shown in muscle fibres of the SM of Brown-Norway rats (male, PND 7, 14, 16, 21, 28, 42 and 84). Consecutive transverse sections are processed by means of the reactions for acid and alkali labile mATPase (Brooke and Kaiser, 1970) and examined by immunoreactivity (IR) with monoclonal antibodies specific to MHC I, MHC IIA, MHC IIX, MHC IIB, Emb MHC, Neo MHC and cardiac- $\alpha$ MHC. All fibres were classified according to protocols used in previous research (Dammeijer et al., 2000).

\subsection{Materials and methods}

\subsubsection{Animals, dissection and tissue processing}

Two EDLs were removed in two inbred strain male Brown-Norway rats of 6 weeks for reference. Two SMs were removed completely in two inbred strain male Brown-Norway rats at PND 7, 14, 16, 21, 28,42 and 84 days. Care for laboratory animals and all animal procedures were in accordance with the Dutch law on animal care ("Wet op de proefdiervoorziening"). The rat was decapitated and the mandible was removed to show the bulla (tympanic cavity equivalent). The caudal wall of the bulla was opened to approach the pyramidal process (containing the SM muscle belly) and the middle ear bones. The lateral bony wall of the pyramidal process, which protrudes in the tympanic cavity, was broken off. SM fibres did not attach to this bony chip. All muscle fibre bundles of the SM were released from the remaining walls of the pyramidal process by blunt dissection. Then, the SM and the stapes were taken out. The stapes was 
cut from the SM tendon before stretching the entire SM (mean length $1.2 \mathrm{~mm}$ ) in a drop of Tissue-tek ${ }^{\circledR}$ (O.C.T. compound) poured between two small wooden matches on a slide. Some SM fibres are arranged oblique or more or less perpendicular to the tendon in situ (Berge and Wirtz, 1989). Stretching of the SM prevents longitudinally cutting of these fibres. Then the specimen was frozen in melting isopentane pre-cooled in liquid nitrogen. The muscles were stored in airtight canisters at $-80^{\circ} \mathrm{C}$.

The entire SMs and parts of the EDL were cut to cross-sections $(10 \mu \mathrm{m})$ on a cryostat microtome at $-20^{\circ} \mathrm{C}$. About 100 SM cross-sections were obtained and numbered from the origin to the tendon. Consecutive cross-sections of the EDL parts were numbered also. One SM and one EDL cross-section were mounted on the same slide. The SM cross-sections numbered 20 up to 60 and accompanying EDL cross-sections were processed by enzymehistochemistry $(\mathrm{EHC})$ or immunohistochemistry $(\mathrm{IHC})$ and consequently they were examined with the microscope. Two levels per SM were chosen for fibre type characterization, near SM cross-section numbers 30 and 50 . The cross-sections of these levels were photographed. A level contains ten consecutive EHC or IHC processed cross-sections. At PND 7, 14, 16, 21, 28, 42 and 84, respectively 2, 3, 2, 4, 6, 3 and 6 levels could be used, each level contained 100 fibres. Not in every muscle that was cut two levels could be used, consequently 1, 2, 1, 2, 3, 2 and 3 muscles were used originating from 1, 2, 1, 2, 2, 2, 2 animals PND 7, 14, 16, 21, 28, 42 and 84 respectively.

\subsection{2 mATPase characterization}

A modification of the procedure of Brooke and Kaiser (1970) was carried out for the acid preincubation method (Ogilvie and Feeback, 1990). Type I fibres stained dark, type IIA fibres stained light, type IIX and IIB fibres presented intermediate staining. The mATPase alkali lability protocol was adapted from Guth and Samaha (1970). The sections were fixed in buffered 2\% methanol-free paraformaldehyde and stained after pre-incubation at pH 10.4. The double pre-incubation method according to Sant'Ana Pereira et al. (1995a) was used to determine fibres which contain myosin type IIX.

Fibres were assigned to different mATPase categories based on combinations of staining intensities (Table 3.1). Myofibrillar ATPase profiles were defined according to Gorza (1990), supplemented with Sant'Ana Pereira's et al. criteria for myosin type IIX (1995a). Fibres that did not meet the criteria of the mATPase categories type I, IIA, IIX or IIB were assigned to type Miscellaneous ('Misc').

Table 3.1 Combinations of fibre staining on mATPase activity after different pre-incubation based on Gorza (1990) and Sant'Ana Pereira et al. (1995a).

\begin{tabular}{llll}
\hline mATPase category & $\mathrm{pH} 4.35$ & $\mathrm{pH} 10.4$ & double pre-incubation \\
\hline type I & ++ (dark) & - (light) & - (light) \\
type IIA & - (light) & ++ (dark) & - (light) \\
type IIX & + (intermediate) & ++ (dark) & $+/++$ (intermediate/dark) \\
type IIB & + (intermediate) & + (intermediate) & - (light) \\
type 'Misc' a & mATPase activity profiles other than above & \\
\hline
\end{tabular}

a Five 'Misc' categories were discerned; two were found at PND 7, three were found at other PND. 


\subsubsection{MHC isoform characterization}

Each fibre categorized along the criteria given in Table 3.1 was further characterized by immunoreactivity (IR), negative (-) and positive (two levels, + or ++ ), on a panel of mAbs, respectively specific to MHC I (219-1D1) (Bredman et al., 1992; Sant'Ana Pereira et al., 1995b), MHC IIA (333-7H1) (Bredman et al., 1992; Sant'Ana Pereira et al., 1995b), IIX (6H1) (Lucas et al., 2000), MHC IIB (MHC BF-F3) (Schiaffino et al., 1989), MHC cardiac- $\alpha$ (249-5A4) (Bredman et al., 1992), Emb MHC (330R5B4) (Bredman et al., 1991) and to Neo MHC (Novocastra). The indirect unconjugated technique after Moorman et al. (1984) was applied. Sections without primary antibody incubation were used as control as well as EDL cross-sections.

\subsubsection{Selection of muscle fibres}

On average 200 fibres were present in each cross-section of the entire muscle. None of the cross-sections processed, by EHC or IHC, showed selectively located muscle fibre staining, which could be indicative for unequal distribution of muscle fibre types. An area that only contained cross-sectioned fibres was selected on the photograph of the cross-section processed for EHC after pre-incubation at $\mathrm{pH}$ 4.35. Then 100 adjacent fibres were numbered in this area. These fibres were identified on the other cross-sections of the given level and numbered correspondingly. A fibre, which could not be found in one of the cross-sections of the level examined was removed from the selection and replaced by an adjacent fibre that showed a staining intensity after pre-incubation at $\mathrm{pH} 4.35$ equal to the excluded fibre. Replacement occurred for about 0-2 fibres per level examined.

Two of the authors independently scored the staining intensity of the selected fibres,-+ or ++ . Scoring was carried out per cross-section. Per level, the score of both examiners of maximally two fibres differed. Assignment of these fibres was established by re-evaluation of the cross-sections in mutual agreement by the two examiners.

\subsection{Results}

The number of SM fibres that could be assigned to mATPase categories type I or IIA according to the criteria mentioned in (Table 3.1) at PND 7 was 72\%. None was assigned to mATPase categories type IIB. All fibres assigned to mATPase type I expressed MHC I and co-expressed Emb MHC.

At this age, $54.5 \%$ of the fibres were assigned to the mATPase category IIA, while none of these fibres expressed MHC IIA. Instead, these expressed Emb MHC (24.5\%) alone or with MHC IIB (30\%). At PND 7 no fibres were classified to the category mATPase type IIX, no IR to the mAb to MHC IIX was noted at PND 7 in any fibre.

At PND 7, 28\% of the fibres were assigned to two mATPase 'Misc' categories (Table 3.3). None of the fibres at other ages were assigned to these categories. Type 'Misc' 1 category contained $13.5 \%$ of fibres which only expressed Emb MHC. 
Table 3.2 SM fibres assigned to mATPase categories type I, type IIA, type IIB and type IIX at PND 7, 14, 16, 21, 28, 42 and 84.

\begin{tabular}{|c|c|c|c|c|c|c|c|c|c|c|c|c|c|}
\hline \multirow{2}{*}{$\begin{array}{l}\text { mATPase } \\
\text { category }\end{array}$} & \multicolumn{6}{|c|}{$m A b I R$} & \multicolumn{7}{|c|}{ Mean \% of fibres at PND: } \\
\hline & $\alpha$ & I & $\| A$ & IIB & IIX & Emb & 7 & 14 & 16 & 21 & 28 & 42 & 84 \\
\hline \multirow[t]{5}{*}{ Type I } & & & & & & & $(17.5)$ & (1) & $(2.5)$ & $(2.5)$ & $(0)$ & $(0.7)$ & (7.7) \\
\hline & - & ++ & - & - & - & - & & & 2.5 & 1 & & & 6.5 \\
\hline & + & ++ & - & - & - & - & & 1 & & 1.5 & & 0.7 & 1.2 \\
\hline & - & ++ & - & - & - & + & 6 & & & & & & \\
\hline & - & ++ & - & - & - & ++ & 11.5 & & & & & & \\
\hline
\end{tabular}

Type IIA

Type IIB

Type IIX

$\begin{array}{lllllll}(54.5) & (15.7) & (11.5) & (13) & (18.2) & (27.7) & (23) \\ & 10.7 & 11.5 & 12.5 & 17.3 & 20.3 & 23 \\ & 4 & & 0.5 & 0.8 & 7.3 & \\ & 1 & & & & & \\ 13 & & & & & & \\ 11.5 & & & & & & \end{array}$

7.5

2

1

16

3.5 $\begin{array}{lllll}(1.7) \quad(1.5) \quad(0.8) \quad(0) \quad(0) \quad(0) & 0\end{array}$

$\begin{array}{llllll} & (57.7) & (59.5) & (61.5) & (60.8) & \text { (54) }\end{array}$

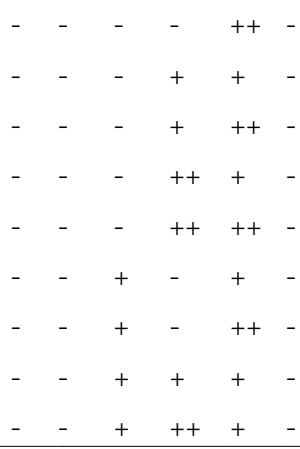

$\begin{array}{lllll}19.7 & 7 & 5.5 & 7 & 1\end{array}$

$\begin{array}{lllll}12.5 & 9 & 11.3 & 14.7 & 1.7\end{array}$

$\begin{array}{llllll}8.3 & 11.5 & 12 & 14.2 & 9 & 0.3\end{array}$

$\begin{array}{llll}3.3 & 2.5 & 2.5 & 1.7\end{array}$

$\begin{array}{llll}2 & 1.5 & 3.5 & 0.5\end{array}$

$\begin{array}{llll}1.3 & 0.5 & 2 & 1\end{array}$

$\begin{array}{lllll}5.3 & 1 & 1.3 & 0.8 & 1.7\end{array}$

2.3

Column 1 of table 3.2 shows the mATPase categories, mean percentages of fibres assigned to a mATPase based category are shown in brackets. The fibres are subdivided to MHC combination groups based on IR to six mAbs against, MHC cardiac- $\alpha(\alpha)$, embryonal MHC (Emb), MHC I, MHC IIA, MHC IIX and MHC IIB. IR to the mAbs used are shown as negative (-), (+) weak and (++) strong. Mean percentages of fibres assigned to a certain MHC combination group are shown in the columns headed by their PND of which the muscle originates. 
Table 3.3 SM fibres assigned to mATPase categories type 'Misc'. at PND 7, 14, 16, 21, 28, 42 and 84.

\begin{tabular}{|c|c|c|c|c|c|c|c|c|c|c|c|c|c|}
\hline \multirow{2}{*}{$\begin{array}{l}\text { mATPase } \\
\text { category }\end{array}$} & \multicolumn{6}{|c|}{$\mathrm{mAb} I \mathrm{R}$} & \multicolumn{7}{|c|}{ Mean \% of fibres at PND: } \\
\hline & $\alpha$ & 1 & $\| A$ & IIB & IIX & $E$ & 7 & 14 & 16 & 21 & 28 & 42 & 84 \\
\hline \multirow[t]{3}{*}{ Type 'Misc' 1} & & & & & & & (13.5) & & & & & & \\
\hline & - & - & - & - & - & + & 4.5 & & & & & & \\
\hline & - & - & - & - & - & ++ & 9 & & & & & & \\
\hline \multirow[t]{4}{*}{ Type 'Misc' 2} & & & & & & & $(14.5)$ & & & & & & \\
\hline & - & + & - & ++ & - & - & 0.5 & & & & & & \\
\hline & - & - & - & + & - & ++ & 1 & & & & & & \\
\hline & - & - & - & ++ & - & + & 13 & & & & & & \\
\hline \multirow[t]{4}{*}{ Type 'Misc' 3} & & & & & & & & $(15.3)$ & $(14)$ & $(10.3)$ & $(13.3)$ & $(15.3)$ & $(10)$ \\
\hline & + & ++ & + & - & - & - & & 2.3 & & 1.5 & 3.5 & 6.3 & 0.7 \\
\hline & - & ++ & + & - & - & - & & 10.7 & 14 & 8.6 & 9.3 & 4.3 & 9.3 \\
\hline & - & ++ & + & + & - & - & & 2.3 & & & 0.5 & 4.7 & \\
\hline \multirow[t]{4}{*}{ Type 'Misc' 4} & & & & & & & & (4) & $(2)$ & $(2)$ & $(2.2)$ & (1) & (0) \\
\hline & - & ++ & - & ++ & + & - & & 0.3 & & 0.5 & & & \\
\hline & - & ++ & - & - & + & - & & 0.3 & & 1.5 & & & \\
\hline & - & ++ & + & - & + & - & & 3.3 & 2 & & 2.2 & 1 & \\
\hline \multirow[t]{4}{*}{ Type 'Misc' 5} & & & & & & & & $(4.7)$ & (9) & (10) & $(5.5)$ & (2.3) & (5.7) \\
\hline & - & - & + & - & + & - & & & 3 & 2.8 & & & \\
\hline & - & - & ++ & - & + & - & & 3 & 4.5 & 6.5 & 5.2 & 2.3 & 5.7 \\
\hline & - & - & ++ & + & + & - & & 1.7 & 1.5 & 0.8 & 0.3 & & \\
\hline
\end{tabular}

The mATPase activity profiles of the categories 'Misc' (staining after pre-incubation pH 4.35, 10.4 or Double preincubation) are 'Misc' (1),,,-+- 2$)+,++,-,(3)++,+,-,(4)++,+,+$ and $(5)-,++,+$. Mean percentages of fibres assigned to a mATPase based category are shown in brackets. The fibres are subdivided to MHC combination groups based on IR to six mAbs against, MHC cardiac- $\alpha(\alpha)$, embryonal MHC (Emb), MHC I, MHC IIA, MHC IIX and MHC IIB. IR to the mAbs used are shown as negative $(-),(+)$ weak and $(++)$ strong.

All fibres in the category mATPase category type 'Misc' 2 showed IR to MHC IIB, 14.5\% of the fibres were assigned to this category. Co-expression with Emb MHC in the majority of these fibres was noted.

The Emb MHC isoform was demonstrated in the SM at PND 7 and 14 only. It was expressed in 98.5\% of the fibres at PND 7, in contrast to 1\% of the fibres at PND 14 (Table 3.2). At PND 7, in 38\% of the fibres Emb MHC was the only MHC that was expressed (Table 3.2 shows $24.5 \%$ and Table 3.3 shows $13.5 \%$ ). Co-expression of the Emb MHC occurred with MHC I (18\%) or MHC IIB (45.5\%), the combination of all three MHCs was not found. In the SM at PND 7 cardiac- $\alpha$ MHC, MHC IIX and MHC IIA could be not demonstrated.

Column 1 of table 3.2 shows the mATPase categories, mean percentages of fibres assigned to a mATPase based category are shown in brackets. The fibres are subdivided to MHC combination groups based on 
IR to six mAbs against, MHC cardiac- $\alpha(\alpha)$, embryonal MHC (Emb), MHC I, MHC IIA, MHC IIX and MHC IIB. IR to the mAbs used are shown as negative (-), (+) weak and (++) strong. Mean percentages of fibres assigned to a certain MHC combination group are shown in the columns headed by their PND of which the muscle originates.

The number of mATPase type I fibres was $17.5 \%$ on PND 7, but then decreased to less than $3 \%$ on PND 14-42 before increasing to $7.7 \%$ on PND 84.

Some of the fibres in this group co-expressed cardiac- $\alpha$ MHC with MHC I, even at PND 84. All SM fibres showing strong (++) IR to the mAb to $\mathrm{MHCI}$ also showed (++) mATPase activity after pre-incubation at pH 4.35 and were assigned to the mATPase category type I or types 'Misc' (3) or 'Misc' (4) (see Table 3.3).

The number of fibres assigned to mATPase type IIA category at PND 14 was 15.7\%, and increased to 23\% at PND 84. All these fibres, in contrast to the situation at PND 7, showed (++) IR to the mAb to MHC IIA. Besides data at PND 7, only at PND 42 a considerable number of the mATPase type IIA fibres co-express for another MHC (mainly MHC IIB, 7.3 \%). At PND 84, all fibres in this category were pure MHC IIA.

Very few fibres could be assigned to mATPase category type IIB, at PND 14, 16 and 21 the number of fibres were $1.7 \%, 1.5 \%$ and $0.8 \%$ respectively.

At all ages, except at PND 7, the majority of SM fibres were assigned to the MATPase type IIX category. The extreme values were a minimum of 53.7\% at PND 84 and maximum $61.5 \%$ at PND 21.

Approximately half of these fibres were MHC hybrids except at the PND 84. At that age, nearly all fibres were pure MHC IIX. In the hybrid fibres co-expression with MHC IIB was seen most often. Few fibres co-expressed MHC IIA in this category, in very few fibres MHC IIX, MHC IIB and MHC IIA were expressed together.

In the SM of PND 14 all MHC isoforms but MHC Neo could be demonstrated. In only a few fibres at PND $14 \mathrm{Emb} \mathrm{MHC}$ was expressed. Except for a few differences the same results were found in the SM fibres 16 days of age. Emb MHC was not shown at 16 days.

The mATPase categories type 'Misc' 3, 'Misc' 4 and 'Misc' 5 were the identified categories of muscle fibres after PND 7. None of the fibres of PND 7 were assigned to these categories.

In the 'Misc' 3 category, all fibres co-expressed MHC I with MHC IIA, a small portion co-expressed cardiac- $\alpha$ MHC or MHC IIB also but none expressed MHC IIX. The number of fibres assigned to this category ranged from a $10 \%$ to $15.3 \%$ of the total fibres, this category seems constantly represented across all ages from PND 14 to PND 84.

Only a few fibres were assigned to mATPase category type 'Misc' 4 and contained fibres with strong IR to the $\mathrm{mAb}$ to $\mathrm{MHC}$ I and forming hybrid MHC expression with MHC IIX, very few also expressing MHC IIA or MHC IIB.

All fibres in the mATPase category type 'Misc' 5 expressed MHC IIA either with IR (+) or (++), all coexpressed MHC IIX to some extent; few also showed co-expression of MHC IIB. The percentages of fibres assigned to this category varied across the different age groups with minimum of $2.3 \%$ at PND 42 and a maximum of 10\% at PND 21. 
The MHC neonatal antibody (Novocastra) used in this study could not produce any immunoreactivity in the SM fibres evaluated.

The number of fibres showing any IR to MHC I ranged from 14.8\% (PND 21) to 20.3\% (PND 14) (fibres were assigned to mATPase categories type I, type 'Misc' 3 and type 'Misc' 4). SM fibres at other ages after PND 7 showed IR to MHC IIA in percentages ranging from 35\% (PND 21) to 50\% (PND 14)( fibres were assigned to mATPase categories type IIA, type IIX, type 'Misc' 3, type 'Misc' 4 and type 'Misc' 5). These percentages showed no clear tendency with respect to increasing age.

The number of fibres showing IR to the mAb to MHC IIB decreased with increasing age, at PND 14, MHC IIB was expressed in 49\% of the SM fibres, compared to only 3\% of SM fibres at PND 84.

In general with increasing age, a tendency towards a decrease of fibres expressing multiple MHC isoforms was noted, such that the percentage of pure MHC type fibres increased with increasing age.

\subsection{Discussion}

Based on the mATPase classification presented here, nine different fibre types could be described. Two of these mATPase fibre types were identified at PND 7 only. IHC by using mAbs against $\mathrm{MHC}$ isoforms identified twenty different fibre types according to their MHC isoform composition. The MHC isoform expressed most dominantly, largely determines to which mATPase category a fibre is assigned to (Sant'Ana Pereira et al., 1995a). This shows the assembly of many MHC based fibre types into one mATPase based fibre type category.

The present study showed that throughout postnatal development the SM of the rat contains, besides a significant number of fibres that express only one MHC, large numbers of fibres that express more than one MHC. We demonstrated that from birth to maturity the number of fibres in the SM expressing only one MHC increased. Up to the age of PND 42, at least half of the SM fibres expressed at least two MHC isoforms. In the SM of PND 84, nearly 80\% of the fibres were "pure" by MHC isoform content, expressing MHC IIA or MHC IIX. The other fibres at this age co-expressed two MHC isoforms, seldom, three. The decrease of the number of MHC hybrid fibres in developing skeletal muscles was shown in other studies (Whalen et al., 1981; Staron and Pette, 1993). It was considered a feature of developing muscles.

The large number of pure MHC fibres at PND 84 seems to weaken the argument stated earlier that the $\mathrm{SM}$, being in a constant MHC hybrid state, is able to adjust to changing environment quickly. MHC hybridism may serve the SM in fine tuning muscle characteristics to changing demands (Dammeijer et al., 2000). In the earlier study, the SM was investigated in rats of six weeks of age. In the present study the SM at PND 42 showed MHC isoform hybridism in more than half of the muscle fibres (although less fibres than in the previous study), the statement still seems valid. The situation at PND 84 may be the result of an ongoing developmental process. It may also reflect the adaptation of the muscle to demands placed upon by the monotonous acoustic environment in the animal facility. 
Although the number of fibres assigned to mATPase type I category fluctuated across the different PNDs, the number of fibres showing IR to the mAb specific to MHC I did not change much at any PND examined. At PND 84 the number of mATPase type I fibres increased, at the same time the number of Types 'Misc' (3) and 'Misc' (4) fibres decreased. In these 'Misc' categories a strong IR to the mAb specific to $\mathrm{MHC}$ I was present as well. The sum of fibres assigned to these three mATPase categories was equal at all ages. These three mATPase categories seem to represent a pool of fibres originating from the same lineage of myogenic precursor cells destined to mainly express the slow, MHC I phenotype. In time a decrease of co-expression of MHC IIA, MHC IIB or MHC IIX in these fibres occurs, as part of development or adjustment to the environment.

Between PND 14 and 28, the number of fibres assigned to mATPase type IIA category increased, after PND 28 the number of these fibres remained constant. Category mATPase IIB fibres ceased to be recognized after PND 21, the number of fibres assigned to this category before this age was very small. The numbers of SM fibres assigned to the mATPase fibre types IIX were stable across the different PNDs after PND 14. We conclude that after some changes between PND 14 and 28, the mATPase based fibre composition of the SM after PND 21 does not change much. After PND 14 the percentages of fibres expressing the different MHC isoforms judged by the IR to the mAbs used was relatively stable. This suggests that the major changes with respect to muscle fibre phenotype development occur in the first three postnatal weeks and thereafter minor changes take place either as a part of a normal development or as a part of adjustment to the environment.

The expression of MHC IIB may seem contradictory to this notion. At PND 14 nearly 50\% of the fibres showed IR to the mAb specific for MHC IIB, this number decreased to only a 3\% of fibres at PND 84. Noteworthy seems to be that the number of fibres assigned to mATPase IIB category was very low at PND 21 and was not identified thereafter, this may question the significance of variation in MHC IIB expression concerning the phenotype of the SM.

The general MHC hybridism in SM fibres at PND 42 as reported in our earlier study (Dammeijer, 2000) was not found. The main reason for hybridism in all fibres was the general MHC IIB expression in that study (Dammeijer, 2000). In the present study, we were not able to reproduce the IR characteristics in any SM that would suggest a general MHC IIB expression in all fibres. In the present study we used an $\mathrm{mAb}$ to MHC IIB from an optimized hybridoma cell line (provided by E. Dupont-Versteegden, Associate Professor Geriatrics and Physiology and Biophysics, Little Rock, USA), which may explain the difference in IR characteristics.

We used Neo MHC antibodies from Novocastra (IgG1, NCL-MHCn) which were also used by others on multiple previous experiments involving rat muscles (Geiger et al., 2001). We found no positive IR to this $\mathrm{mAb}$ in the SMs. Moreover, we tried to show IR in diaphragm muscle fibres of the Brown-Norway rat at PND 7 too, but none were found. Rabbit and human masseter muscles were used as reference and did show positive fibres clearly. Immunohistochemical techniques used were checked by performing the staining for neonatal MHC on the different types of muscles at another laboratory (laboratory of the 
University of Amsterdam, by J.A.M. Korfage), the same results were found. Therefore we conclude that the histochemical technique used was conformed to the standard procedure and that neonatal $\mathrm{MHC}$ can not be shown in the rat SM by the mAb we used.

Neo MHC was shown in muscles of very young rats up to the age of 40 days (Di Maso et al. 2000), it was concluded that the regulation of the Neo MHC and fast type IIB MHC isoforms are coupled to one another as a down regulation of Neo MHC coincides with an up regulation of MHC IIB. The SM fibres already show an IR to the mAb specific to MHC IIB at PND 7, which seems very early, whether Neo MHC IR could have been presented at an earlier developmental period remains speculative.

This study shows that at PND 7 nearly all fibres still express the developmental embryonic MHC, coexpression with $\mathrm{MHCI}$ and IIB in 18\% and $44.5 \%$ of the fibres respectively. Rat hind limb skeletal muscle was shown to be in an undifferentiated state after birth too, besides embryonal and neonatal MHC isoforms, MHC I was expressed at PND 7 with only trace amounts of the adult IIB and MHC IIX (Adams et al., 1999). The presence of MHC IIB in almost half of the SM fibres at PND 7 may suggest a precocious development of this muscle already at birth.

In the study by Adams et al. (1999), it was shown that in the soleus and plantaris 30 days of postnatal development were necessary for the attainment of the adult contractile protein phenotype. Our findings indicate that within the first 2 weeks after birth all SM fibres express adult MHC isoforms, like IIB, IIA and IIX accompanied by a near complete disappearance of embryonic MHC. This conversion is fast in comparison with diaphragm muscle fibres, as in the rat diaphragm 28 days were needed before the transition into the adult type MHC was reported to be complete (Johnson et al., 1994). A difference between the stapedius and the other muscles discussed may be that the stapedius reaches its adult size by day 21 but that the other muscles investigated will continue to grow until the animal reaches adult size. Whether this restricted growth is one contribute to the precocial development of the stapedius is not clear.

In the rat, the onset of auditory function as monitored by recording ABR was reported to begin on PND 12-14 and reaches near adult thresholds at about PND 22 (Geal-Dor et al., 1993). This onset of auditory function coincides with the complete pneumatization of the neonatal middle ear, at the beginning of the third postnatal week. At this time the proposed function of the SM, protecting the inner ear against acoustic trauma, is supposed to be appropriate.

\subsection{Conclusions}

In the SM in the rat the adult like MHC isoform composition develops rapidly, even faster than in diaphragm and skeletal muscles. Transition of the MHCs in the SM muscle fibres into the adult MHCs is accomplished at PND 21 as well as from PND 21 the overall mATPase based fibre classification composition remains almost the same. This suggests that the near adult muscle phenotype composition 
is accomplished in the third postnatal week. This development runs synchronously with the onset of auditory function at the same postnatal moment.

From PND 21 up to PND 84 MHC hybridism decreases, which may be an expression of structurally consolidating the adult functional state reached at PND 21 and making it definitive. It should be investigated whether the definitive state of the SM still shows MHC composition plasticity on demand of variation of functioning.

Acknowledgements: We kindly thank A.F.M. Moorman, Professor Dept. Anatomy/Embryology, University of Amsterdam, and E. Dupont-Versteegden, Associate Professor Geriatrics and Physiology and Biophysics, Little Rock, USA, for the possibility of using the mAbs deployed. We kindly thank J. A. M. Korfage of the University of Amsterdam for the preparation of tissue samples for reference purposes. 


\section{References}

- Adams, G.R., McCue, S. A., Zeng, M., Baldwin, K.M., 1999. Time course of myosin heavy chain transitions in neonatal rats: importance of innervation and thyroid state. Am. J. Physiol. Regul. Integr. Comp. Physiol., 276, 954-961.

- Agbulut, O., Noirez, P., Beaumont, F., Butler-Browne, G., 2003. Myosin heavy chain isoforms in postnatal muscle development of mice. Biol. Cell., 95(6), 399-406.

- $\quad$ Berge, H. van den, Kingma, H., Kluge, C., Marres, E.H.M.A., 1990. Electrophysiological aspects of the middle ear muscle reflex in the rat: Latency, rise time and effect on sound transmission. Hear. Res. 48, 209-219.

- Berge, H. van den, Wirtz, P., 1989. Detailed morphology of the stapedius muscle of the rat. J. Anat. 166, 157-169.

- Borg, E., Zakrisson, J.E., 1975. The activity of the stapedius muscle in man during vocalization. Acta Otolaryngol. 79(5-6), 325333.

- Borg, E., Counter, S.A., Rosler, G., 1984. Theories about middle ear muscle function. In: Silman, S. (Ed.), The acoustic reflex; Basic Principles and Clinical Applications. Acad. Press, Orlando Fl, pp. 50-87.

- Bredman, J.J., Wessels, A., Weijs, W.A., Korfage, J.A., Soffers, C.A., Moorman, A.F., 1991. Demonstration of cardiac-specific myosin heavy chain in masticatory muscles of human and rabbit. Histochem. J. 23, 160-170.

- Bredman, J.J., Weijs, W.A., Moorman, A.F.M., 1992. Presence of cardiac $\alpha$-myosin correlates with histochemical myosin Ca ${ }^{2+}$ ATPase activity in the rabbit masseter muscle. Histochem. J. 24, 260-265.

- $\quad$ Brooke, M.H., Kaiser, K.K., 1970. Muscle fibre types how many and what kind? Arch. Neurol. 23, 369-379.

- Butler-Browne, G.S., Whalen, R.G., 1984. Myosin isozyme transitions occurring during the postnatal development of the rat soleus muscle. Dev. Biol. 102(2), 324-34.

- d'Albis, A., Couteaux, R., Janmot, C., Roulet, A., 1989. Specific programs of myosin expression in the postnatal development of rat muscles. Eur. J. Biochem. 183(3), 583-590.

- Dammeijer, P.F.M., van Mameren, H., van Dijk, P., Moorman, A.F.M, Habets, P., Manni, J.J., Drukker, J., 2000. Stapedius muscle fibre composition in the rat. Hear. Res. 141(1-2), 169-79.

- Di Maso, N.A., Caiozzo, V.J., Baldwin, K.M., 2000. Single-fiber myosin heavy chain polymorphism during postnatal development: modulation by hypothyroidism. Am. J. Physiol. Regul. Integr. Comp. Physiol. 278(4), R1099-1106.

- $\quad$ Fletcher, J.L., Riopelle, A.J., 1960. The protective effect of the acoustic reflex for impulsive noise. J. Acoust. Soc. Am. 32, 401404.

- Geal-Dor, M., Freeman, S., Li, G., Sohmer, H., 1993. Development of hearing in neonatal rats: air and bone conducted ABR thresholds. Hear. Res. 69, 236-242.

- Geiger, P.C., Cody, M.J., Macken, R.L., Bayrd, M., E., Fang, Y., Sieck, G.C., 2001. Mechanisms underlying increased force generation by rat diaphragm muscle fibers during development. J. Appl. Physiol. 90, 380-388.

- Gorza, L., 1990. Identification of a novel type 2 fibre population in mammalian skeletal muscle by combined use of histochemical myosin ATPase and anti-myosin monoclonal antibodies. J. Histochem. Cytochem. 38, 257-265.

- Guth, L., Samaha, F.J., 1970. Procedure for the histochemical demonstration of actomyosin ATPase. Exp. Neurol. 28, 365-367.

- Hoh, J.F.Y., 1992. Muscle fiber types and function. Curr. Opin. Rheumatol. 4, 801-808.

- Johnson, B.D., Wilson, L.E., Zhan, W.Z., Watchko, J.F., Daood, M.J., Sieck, G.C., 1994. Contractile properties of the developing diaphragm correlate with myosin heavy chain phenotype. J. Appl. Physiol. 77, 481-487. 
- Lucas, C.A., Kang, L.H., Hoh, J.F., 2000. Monospecific Antibodies against the Three Mammalian Fast Limb Myosin Heavy Chains. Biochem. Biophys. Res. Commun. 272, 303-308.

- Møller, A.R., 1965. An experimental study of the acoustic impedance of the middle ear and its transmission properties. Acta Otolaryngol. 60, 129-149.

- Moorman, A.F.M., Boer, P.A.J. de, Linders, M.Th., Charles, R., 1984. The histone H5 variant in Xenopus laevis. Cell. Differ. 14, 113123.

- $\quad$ Ogilvie, R.W., Feeback, D.L., 1990. A metachromatic dye-ATPase method for the simultaneous identification of skeletal muscle fibre types I, IIA, IIB and IIC. Stain technol. 65, 231-241.

- Ono, J., Nagel, D., Pfalz, R., 1980. The human stapedius reflex by ipsilateral electrical stimulation of the auditory meatal skin. HNO 28(3), 104-107.

- Pang, X.D., Guinan, J.J., 1997. Effects of stapedius-muscle contractions on the masking of auditory-nerve responses. J. Acoust. Soc. Am. 102(6), 3576-3586.

- $\quad$ Pette, D., Staron, R.S., 2000. Myosin isoforms, muscle fiber types, and transitions. Microsc. Res. Tech. 50(6), 500-509.

- Perie, S., Agbulut, O., St Guily, J.L., Butler-Browne, G.S., 2000. Myosin heavy chain expression in human laryngeal muscle fibers. A biochemical study. Ann. Otol. Rhinol. Laryngol. 109(2), 216-220.

- Pilz, P.K., Ostwald, J., Kreiter, A., Schnitzler, H.U., 1997. Effect of the middle ear reflex on sound transmission to the inner ear of rat. Hear. Res. 105(1-2), 171-82.

- Sant'Ana Pereira, J.A.A., Haan, de A., Wessels, A., Moorman, A.F.M., Sargeant, A.J., 1995a. The mATPase histochemical profile of rat type IIX fibres: Correlation with MHC immunolabelling. Histochem. J. 27, 715-722.

- Sant'Ana Pereira, J.A.A., Wessels, A., Nijtmans, L., Moorman, A.F.M., Sargeant, A.J., 1995b. New method for the accurate characterisation of single human skeletal muscle fibres demonstrates a relation between mATPase and MHC expression in pure and hybrid fibre types. J. Muscle Res. Cell Motility 16, 21-34.

- Schiaffino, S., Gorza, L., Sartore, S., Saggin, L., Ausini, S., Vianello, M., Gundersen, K., Lomo, T., 1989. Three myosin heavy chain isoforms in type 2 skeletal muscle fibres. J. Muscle Res. Cell Motility 10, 197-205.

- $\quad$ Schiaffino, S., Gorza, L., Ausoni, S., Bottinelli, R., Reggaini, C., Edstrom, L., Larsen, L., Gundersen, K., Lomo, T., 1990. Muscle fiber types expressing different myosin heavy chain isoforms. Their functional properties and adaptive capacity. In: Pette, D. (Ed), The Dynamic State of Muscle Fibers. deGruyter, New York, pp. 329-341.

- Schiaffino, S., Reggiani, C., 1996. Molecular diversity of myofibrillar proteins: gene regulation and functional significance. Physiol. Rev. 76(2), 371-423.

- Staron, R.C., Pette, D., 1993. The continuum of pure and hybrid myosin heavy chain based fibre types in rat skeletal muscles. Histochemistry 100, 149-153.

- Usami, A., Abe, S., Ide, Y., 2003. Myosin heavy chain isoforms of the murine masseter muscle during pre- and post-natal development. Anat. Histol. Embryol. 32(4), 244-248.

- Whalen, R.G., Sell, S.M., Butler-Browne, G.S., 1981. Three myosin heavy-chain isozymes appear sequentially in rat muscle development. Nature 292, 805-809.

- Wormald, P.J., Rogers, C., Gatehouse, S., 1995. Speech discrimination in patients with Bell's palsy and a paralysed stapedius muscle. Clin. Otolaryngol. 20(1), 59-62. 


\section{Chapter 3}

- Wieczorek, D.F., Periasamy, M., Butler-Browne, G.S., Whalen, R.G., Nadal-Ginard, B., 1985. Co-expression of multiple myosin heavy chain genes, in addition to a tissue-specific one, in extraocular musculature. J. Cell Biol. 101(2), 618-29.

- Zakrisson, J.E., 1975. The role of the stapedius reflex in post-stimulatory auditory fatigue. Acta Otolaryngol. 79, 1-10. 


\section{Chapter 4}

\section{Effects of early auditory deprivation and stimulation on auditory brainstem responses in the rat}

Patrick F.M. Dammeijer, Quila C.M. Schlundt Bodien, Michelene N. Chenault, Johannes J. Manni, Lucien J.C. Anteunis

Department of Otorhinolaryngology and Head and Neck Surgery University Hospital Maastricht The Netherlands

Published in Acta Otolaryngologica 2002; 122(7), 703-708. 


\begin{abstract}
The purpose of this study was to investigate the effect of auditory sound deprivation or stimulation on auditory brain stem responses (ABRs) during the maturation period of the rat auditory system. At postnatal day (PND) 21, 40 newborn Norway-Brown male rats were categorized into 3 groups: (i) an auditory deprivation group in which a bilateral average conductive hearing loss of $27 \mathrm{~dB}$ was induced, (ii) an auditory activation group exposed to 65-90 dB sound pressure level; and (iii) a control group. ABR recordings were made on PND 84. In order to compare group differences in interpeak latency (IPL), sensation level (SL), defined as stimulus intensity above threshold, was used. IPL measurements and analysis were restricted to the 20-60 dB SL range. No differences were observed in the IPLs of peaks I-IV between the three groups. Small, but not statistically significant, differences in mean estimated IPL of peaks I-IV were shown in the ranges $>50 \mathrm{~dB}$ SL and $<25 \mathrm{~dB}$ SL. Possible confounding factors explaining the apparent discrepancy between these results and those of other animal studies are reviewed.
\end{abstract}




\subsection{Introduction}

Among the factors most commonly invoked to explain maturational changes in auditory evoked potentials are progressive myelinization of fibre tracts, fibre diameter growth, facilitation of synaptic transmission and improved synchronisation of the afferent volley (see Shaw, 1988, for a review). Friauf and Lohmann (1999) summarized evidence for both activity-independent and -dependent processes involved in the generation of the auditory brainstem circuitry of birds and mammals. Although the emergence of crude topography is less activity-dependent, neuronal activity supports cell survival and affects dendritic and axonal growth and is therefore necessary for proper formation of neuronal circuitry.

Deprivation of auditory input probably leads to a retardation of the myelinization process. Absence of auditory stimuli may even lead to degenerative effects. Morphological changes have been described to occur, for example, in sound deprived mice (Webster and Webster, 1977) and rats (Blatchley et al., 1983). These effects were reviewed by Moore (1985); such morphological changes can then lead to impaired development of the Auditory Brainstem Responses (ABR), with consequently longer latencies. Several studies showed prolongation of the ABR latencies in sound-deprived guinea pigs (Walger et al., 1993) and in rats (Keilmann, 1993). Overall, it seems that the effect of auditory deprivation depends largely on the age at onset. In this respect, one considers the period during which this development is sensitive to external conditions as the sensitive period (Eggermont, 1986). In contrast, a critical period is defined as a period during which the action of a specific condition is required for normal development of the system; if disturbed the effect seems largely irreversible.

In comparing the effects of auditory deprivation or stimulation between different species and the consequence of the time of onset, one needs to consider whether the species has an immature auditory system. The term altricial and precocial are used if the species has an immature or mature system at birth, respectively. Shortly after birth the auditory system of the altricious species is probably more vulnerable to deprivation than that of the precocious species (Eggermont, 1986).

If auditory deprivation is to have any effect on maturation of the neural pathways, both the middle ear and cochlea need to have achieved at least a functional status (Saunders et al., 1993). In the rat the onset of auditory function as monitored by recording ABR was reported to begin on post-natal day (PND) 12-14 and reaches near adult thresholds at $\approx 22$ days (Geal Dor et al., 1993). In studies concerning development of ABR in the Mongolian gerbil (McFadden et al., 1996) and the cat (Walsh et al., 1986), both of which are altricious species like the rat, three phases were identified based on the appearance of frequency threshold curves. The first period was characterized by insensitivity, at the end of which ABRs could only be elicited by intense stimuli (95-110 dB sound pressure level (SPL)). During the second period thresholds improved dramatically to near-adult values and during the third period, starting at 
PND 13-14, thresholds improved steadily to adult-like values. Maturation of the conductive middle ear apparatus and cochlea seem to be the major contributing factor to the rapid improvement in thresholds during the relatively short second stage (Geal Dor et al., 1993; Walsh et al., 1986; Woolf and Ryan, 1988; Doan et al., 1996). Threshold improvement during the third stage seems to depend primarily on further maturation of the neural circuitry.

Little is known about the influence of stimulation on the maturation process. From the study in rats of Walsh (1981), it appeared that animals reared in environments rich in sensory stimulation showed greater cerebral weight and length and cortical depth compared with their stimulus-deprived counterparts. Duck-embryos exposed to enhanced species-specific acoustic components of the maternal call, showed an accelerated decline in ABR thresholds and latencies of peak 1 (P1) (Dmitrieva and Gottlieb, 1994). Auditory stimulation during the maturation period may thus lead to an accelerated development, reflected by decreasing $A B R$ latencies and electrophysiological thresholds at lower intensities.

In the present study, the onset of deprivation and stimulation occurred towards the end of the maturation process in the rat. It started at PND 21, when the middle ear and cochlea are functional and ABR threshold is at near-adult values. The period chosen was more comparable to the situation in the human neonate than that of the earlier stages of maturation of the rat. It has been shown that there are significantly longer absolute and interwave latencies in young children with a positive history of otitis media with effusion (OME) in early life, when compared to an age-matched group with a negative OME history (Anteunis and Engel, 2000; Folsom et al., 1983). It is not known whether in the rat, this relatively late maturational stage still constitutes a period during which maturation is sensitive to auditory deprivation or stimulation.

The purpose of the study was to investigate the effect of auditory sound deprivation and stimulation on ABRs towards the end of the maturation period of the auditory system of male Norway-Brown rats. This investigation constitutes part of a larger study concerning the morphological changes that occur in the middle ear muscles after either auditory stimulation or deprivation (Dammeijer et al., 2000). Acquiring these data was considered necessary in order to quantify the sound deadening effect of meatal closure and to exclude the possibility that the auditory stimulus caused any cochlear damage.

We postulated that auditory deprivation, because of its negative influence on the maturation process, would lead to an inhibited shortening of the absolute peak latencies, resulting in a longer brain stem transmission time (BSTT), as reflected in the interpeak latency (IPL). In contrast, auditory stimulation would accelerate the maturation process, resulting in a shortening of the BST. 


\subsection{Materials and methods}

\subsubsection{Animals and procedures}

Forty newborn Norway-Brown male rats were raised with their mothers in cages with food and water available ad libitum and kept on a 12-h light/dark cycle until PND 18, when the mothers were removed from the quarters. On PND 21, the rats were categorized into the following groups: an auditory deprivation group, an auditory activation group and a control group.

In the auditory deprivation group $(n=15)$, conductive hearing loss was produced by closure of the external meatus at PND 21. The animals were anaesthetized using ketamin $(1.3 \mathrm{ml} / \mathrm{kg}$ i.m.) and xylazine $(0.1 \mathrm{ml}$ i.m.) in combination. Both ear canals were obliterated by incising the skin near the tympanic membrane and then elevating the meatal skin towards the pinnae. The skinflaps were approximated using vicryl 5.0 sutures (Ethicon ${ }^{\circledR}$ ). During the experiment the ears were inspected weekly; no signs of infection were noted.

The auditory activation group $(n=12)$ received enhanced exposure to $1 \mathrm{~s}$ narrow band noise/ $1 \mathrm{~s}$ silence at random frequencies (range: $0.1-12 \mathrm{kHz}$ and $65-90 \mathrm{~dB} \mathrm{SPL}$ ) for a period of 9 weeks on a 12-h enhanced exposure / ambient noise cycle.

The control group ( $n=13$ ) was raised in normal animal quarters at normal ambient noise levels ( $60 \mathrm{~dB}$ $\mathrm{SPL}$ in the $0.01-20 \mathrm{kHz}$ range).

After the electrophysiological recordings, the rats were decapitated and the middle ears were opened to remove the stapedius and the tensor tympani muscles for other purposes as described in the Introduction. Normal anatomy of the middle ear was seen in all animals. The care of the laboratory animals and all animal procedures were in accordance with the Dutch law on animal care.

\subsubsection{Electrophysiologic recordings}

ABRs were recorded on PND 84 in a manner similar to that described by van den Berge et al. (1990). The animals were anaesthetized using ketamin ( $1.3 \mathrm{ml} / \mathrm{kg}$ i.m.) and xylazine $(0.1 \mathrm{ml}$ i.m.) in combination before testing and placed in a double-walled, sound-isolated Faraday room. Normothermia was maintained during the $\approx 30$-min recording sessions using a heating blanket coupled to a feedback system with a rectal probe.

Needle electrodes were placed subdermally at scalp-midline (positive), inferior to the stimulated ear (negative) and 1-2 cm in the midline of the back posterior to the neck (reference).

Auditory stimulation consisted of rarefaction clicks of $100-\mu$ s duration with intensities varying from $90 \mathrm{~dB}$ peak equivalent (pe) SPL down to response-threshold, at a rate of $11.7 \mathrm{~Hz}$ produced by a Nicolet CA 2000 instrument and delivered to Nicolet Model TIP-300 transducers with a tube length of $25 \mathrm{~cm}$ (stimulus parameters refer to the output characteristics of the transducer tubes). The end of the tube was inserted in the outer ear canal and a tight seal was obtained. 
Bioelectric activity was amplified and recorded using the Nicolet CA 2000 instrument (band-pass filter $30-3,000 \mathrm{~Hz}$ ). ABRs were obtained by averaging the electroencephalographic signal to $500-1,000$ clicks (time window $20 \mathrm{~ms}$; sampling frequency $50 \mathrm{kHz}$ ). Fifty- $\mathrm{Hz}$ notch filtering was employed and contaminated epochs were eliminated by artefact rejection.

The recording of the ABR in rats resulted in a series of four identifiable waves. Waves II and III were often seen as a complex, in which the waves were sometimes less clearly separable. Wave IV remained the most consistently observed response at near-threshold level. Threshold was defined as the lowest intensity level that elicited a reproducible response.

\subsubsection{Data analysis}

The relation between $\mathrm{dB}$ stimulus levels and IPLs was investigated for the three groups. Group differences in IPLs were also compared per sensation level (SL), defined as stimulus intensity above threshold. SL was used in order to compensate for individual differences in hearing sensation and for the influence of SL on IPLs (Sohmer and Friedman, 1992; Stockard et al., 1979). IPL measurements and analysis were restricted to the $20-60 \mathrm{~dB}$ SL range.

Repeated measure analysis with group as between-subject factor and SL as within-subject factor was performed. When an unexpected discrepancy was encountered, namely that the IPL for the stimulated group was larger than that for the control group, and because it is tenable that threshold or hearing loss influences latency, the repeated measure analysis was repeated, correcting for threshold.

The three SLs adhered to for the control group were used as a basis. Although the SLs of the other two groups showed some deviation from these reference values, because readings were not consistently obtainable at the exact same values, the ranges were within $5 \mathrm{~dB}$ of the SLs defined.

\subsection{Results}

\subsubsection{Thresholds}

The mean click threshold was $0.38 \mathrm{~dB}$ peSPL (SD 1.39) in the control group, $29.64 \mathrm{~dB}$ peSPL (SD 6.64) in the auditory deprived group, and $0.42 \mathrm{~dB}$ peSPL (SD 3.34) in the stimulated group, indicating a mean conductive hearing loss of almost $30 \mathrm{~dB}$ in the auditory deprivation group.

\subsubsection{Latencies}

With decreasing click intensity, absolute latencies increased in all groups. Fig. 4.1. shows the IPLs for four unadjusted dB stimulation levels. Not all the deprived animals showed responses at the lower levels, which can be seen at the $25 \mathrm{~dB}$ level, were values were only obtained for 4 rats. Furthermore, the graph illustrates an apparent shift in the IPLs, which is presumably a result of the conductive hearing loss of $\approx 30 \mathrm{~dB}$ (Stockard et al., 1979). 


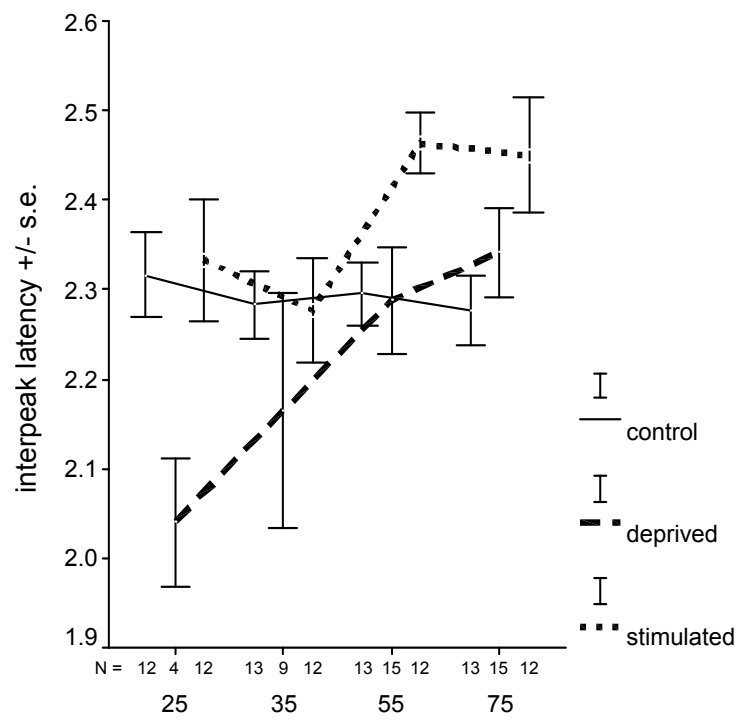

$\mathrm{dB}$

Fig. 4.1. Mean estimated IPL I-IV as a function of $d B$ stimulus level

Table 4.1 shows the mean estimated peaks I and IV and the IPL of peaks I-IV (IPL I-IV) at three levels of stimulation. There is clearly a negative linear relation between stimulation and peak latencies. Repeated measures analysis demonstrated that stimulation level and group were statistically significant factors for all four peak latencies. Significant differences between the deprived group and the control group were only present at lower SLs for the estimated P1 $(p<0.01)$ and P3 $(p<0.01)$ latencies. For the estimated P2 and P4 latency values, no significant differences were observed between the three groups, suggesting no effect of either deprivation or stimulation.

Despite the SL being compensated for the conductive hearing loss, the auditory deprived group showed longer absolute P1 and P4 latencies compared to the control and the stimulated groups. The control group showed the lowest P1 and P4 latencies.

A repeated measures ANOVA was performed with the three groups as between-subject factor and the three SLs as repeated measures (within-subject factor). An adjustment for the unequal spacing of the SLs was included. The assumptions of sphericity and normality were met. There was no statistically significant difference between the groups ( $p=0.651)$, but there was a difference in IPL between the three SLS $(p<$ 0.0005). There was a statistically significant interaction effect between group and SL ( $p=0.026$ ).

Fig. 4.2 shows that small, but not statistically significant, differences were visible in the ranges $>50 \mathrm{~dB}$ $\mathrm{SL}$ and $<25 \mathrm{~dB}$ SL. The mean estimated IPL of the deprived group showed the same course as that of the stimulated group, namely an increase in both border ranges (lowest and highest ranges of SL). The 
Table 4.1 Mean estimated peaks I and IV and IPL I-IV at three levels of stimulation

\begin{tabular}{|c|c|c|c|c|c|c|c|c|c|}
\hline \multirow[b]{3}{*}{ Group } & \multicolumn{9}{|l|}{$\mathrm{SL}(\mathrm{dB})$} \\
\hline & \multicolumn{3}{|l|}{25} & \multicolumn{3}{|l|}{35} & \multicolumn{3}{|l|}{55} \\
\hline & Peak I & Peak IV & IPL I-IV & Peak I & Peak IV & IPL I-V & Peak I & Peak IV & IPL I-IV \\
\hline \multicolumn{10}{|c|}{ Control } \\
\hline Mean & 1.78 & 4.10 & 2.32 & 1.68 & 3.94 & 2.27 & 1.49 & 3.79 & 2.30 \\
\hline SE & 0.06 & 0.05 & 0.05 & 0.05 & 0.04 & 0.04 & 0.01 & 0.04 & 0.04 \\
\hline$n$ & 13 & 13 & 13 & 12 & 13 & 12 & 13 & 13 & 13 \\
\hline \multicolumn{10}{|c|}{ Deprived } \\
\hline Mean & 2.49 & 4.78 & 2.29 & 2.15 & 4.44 & 2.29 & 1.87 & 4.27 & 2.40 \\
\hline SE & 0.09 & 0.07 & 0.06 & 0.07 & 0.07 & 0.04 & 0.06 & 0.06 & 0.03 \\
\hline$n$ & 15 & 15 & 15 & 15 & 15 & 15 & 14 & 14 & 14 \\
\hline \multicolumn{10}{|c|}{ Stimulated } \\
\hline Mean & 2.13 & 4.42 & 2.29 & 2.03 & 4.31 & 2.28 & 1.64 & 4.11 & 2.46 \\
\hline SE & 0.04 & 0.05 & 0.06 & 0.03 & 0.08 & 0.06 & 0.04 & 0.06 & 0.03 \\
\hline$n$ & 11 & 11 & 11 & 12 & 12 & 12 & 12 & 12 & 12 \\
\hline
\end{tabular}

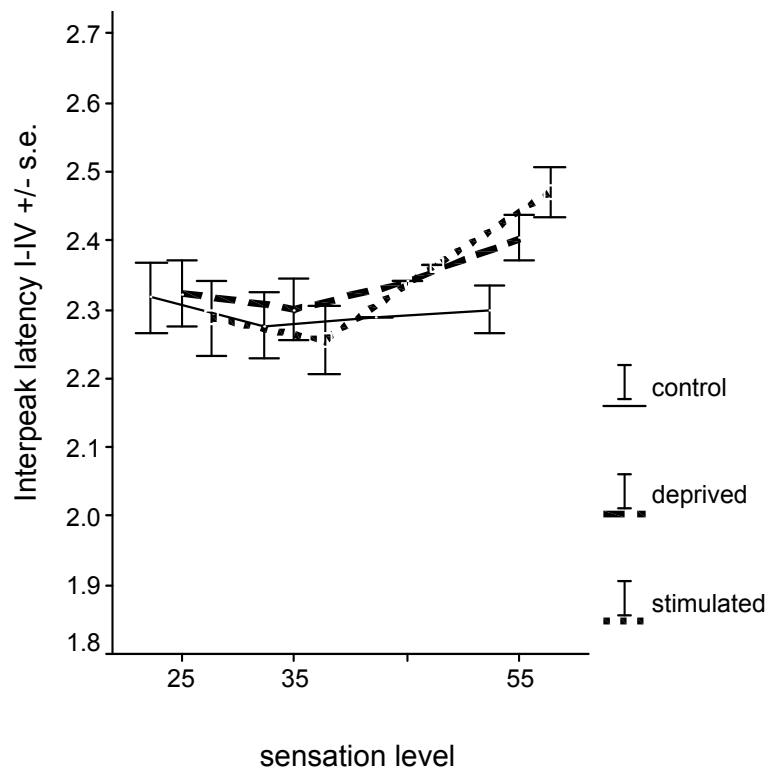

Fig. 4.2. Mean estimated IPL I-IV as a function of SL 


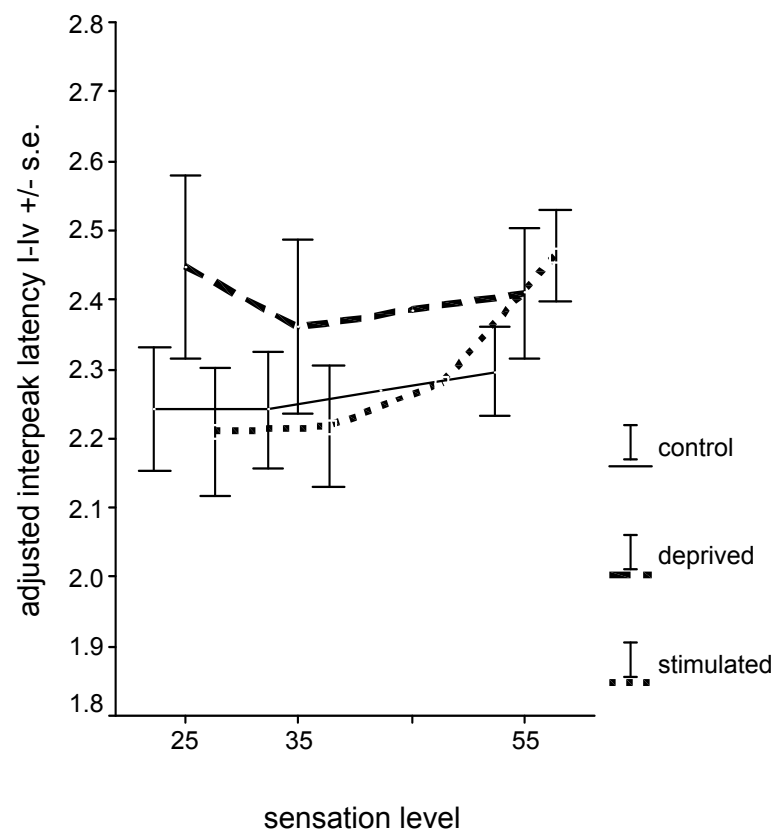

Fig. 4.3. Mean estimated IPL I-IV as a function of SL corrected for threshold.

stimulation group showed an increasing IPL with increasing $S L$, achieving the longest IPL I-IV at $55 \mathrm{~dB}$ SL.

Because threshold or amount of deprivation should have an influence on latencies we performed the statistical analysis mentioned above, correcting for threshold. Again there was no statistical difference between the groups. The difference between the SLs was now no longer statistically significant but there was still an interaction effect between group and sensation level $(p=0.020)$. Fig. 4.3 illustrates the lack of any significant difference in IPL I-IV between the three groups and therefore no treatment effect.

\subsection{Discussion}

In this study the ear canals of the auditory deprivation group were surgically closed at PND 21, a time when ABRs have been reported to reach near-adult threshold (Geal Dor et al., 1993). By closing the ear canals a conductive hearing loss was induced which in a way was similar to reduction of the stimulus intensity, because sound energy is decreased by the time it reaches the cochlea. A bilateral average conductive hearing loss of 29.64 SPL (SD 6.64) was achieved. The amount of deprivation attained was comparable to that described by others (Keilmann, 1993; Walger et al., 1993). 
In our study we not only considered the effect of SL but in addition, IPL measurements and analysis were restricted to the 20-60 dB SL range in order to avoid as far as possible naturally occurring variation in IPL I- $V$ when stimulating below $20 \mathrm{~dB}$ or above $60 \mathrm{~dB}$ SL. IPLs obtained at $\leq 20 \mathrm{~dB}$ SL seriously compromise the significance of apparent differences in mean IPL between experimental groups. Keilmann (1993) found that the IPLs I-V were significantly prolonged between the PNDs 24 and 36, when stimulating only $20 \mathrm{~dB}$ above threshold. Walger et al. (1993) stated that the development of click-evoked ABR IPLs was retarded when analysed at $20 \mathrm{~dB}$ above threshold. In studies by Sohmer and co-workers (Sohmer et al., 1991; Sohmer and Friedman, 1992) conducted in rat pups the IPLs were measured at $80 \mathrm{~dB}$ SL. The differences between our results and those from the other auditory deprivation studies mentioned may be due to varying stimuli and auditory deprivation periods.

The results of our study failed to demonstrate that auditory deprivation starting at PND 21 and lasting for 9 weeks has a statistical significant effect on the IPL I-IV. Absolute IPL was larger in the deprived group than the control group by an amount comparable to the differences reported in the study of Walger et al. (1993). However contrary to our findings, and although the data in that study only involved 12 ears in 6 rats per group, their statistical analysis suggested that the difference was significant.

The importance of the appropriate choice of statistical method in analysing the effect on IPL is shown in Fig. 4.2. As the degree and duration of auditory deprivation ought to have effect on latencies, the analysis should include a correction for threshold. Apparent differences in IPL are hereby diminished.

One study in rhesus monkeys also did not find any significant change in ABR with neonatal conductive hearing loss (Doyle and Webster, 1991), in contrast to results reported in rats and guinea pigs. The speciesassociated difference could be explained by the fact that mice and rats are auditorily altricial, while monkeys are precocial; hence the monkey's auditory maturation is assumed to be less likely influenced by deprivation. In this respect it is even more surprising that in children (also auditory precocial) with a positive history of OME in early life, significantly longer absolute and interwave latencies were found compared to an age-matched group with a negative OME history (Anteunis and Engel, 2000; Folsom et al., 1983). Evidently, species-associated differences in this respect seem to be determined not just by auditory maturation, whether altricial or precocial.

In evoked potential studies, the anaesthetic agent can also be a potentially confounding factor. Based on previous study experience, we assume that anaesthesia with ketamin-xylazine mixture used in this study did not affect the ABR. Ketamin-xylazine seems to be a better agent for evoked potential monitoring purposes, because it produces less pronounced and shorter lasting effects on responses compared with pentobarbital (Goss Sampson and Kriss, 1991). Keilmann (1993) and Walger et al. (1993) used pentobarbital.

We deprived the rats binaurally from PND 21. A possible explanation for the discrepancy between our results with those of Walger et al. (1993) and Keilmann (1993) is that the start of binaural deprivation in their studies occurred immediately after birth. The experiment of Sohmer and Friedman (1992) started at 
PND 9. We started deprivation on PND 21, when most of the maturation may have already taken place (Shaw, 1988). Therefore, deprivation might not have had any prominent effect on the ABR of the rats. Exposing rats to click-stimuli for 9 weeks from PND 21 onwards did not have any effect on the ABR recordings, as the recordings were not significantly different from those in the control group. The maturation of the central auditory pathways after PND 21 is apparently insensitive to this kind of stimulus. For the benefit of further research, we note that this stimulus did not cause any cochlear damage.

It remains unclear which mechanism precisely underlies the absence of difference in ABR between the three groups in our study.

Acknowledgements: This research was supported by The Heinsius Houbolt Foundation and the Maastricht ENT Research Foundation. 


\section{References}

- Anteunis, L.J.C., Engel, J.A.M., 2000. Maastricht Otitis Media with Effusion Study, a prospective longitudinal study in infants from 0 to 2 years. Thesis, University of Limburg, The Netherlands, pp. 179-193.

- Berge, van den H, Kingma, H., Kluge, C., Marres, E.H.M.A., 1990. Electrophysiological aspects of the middle ear muscle reflex in the rat: Latency, rise time and effect on sound transmission. Hear. Res. 48, 209-219.

- Blatchley, B.J., Williams, J.E., Coleman, J.R., 1983. Age-dependent effects of acoustic deprivation on spherical cells of the rat anteroventral cochlear nucleus. Exp. Neurol. 80, 81-93.

- Dammeijer, P.F.M., Mameren, van H., Dijk, van P., Moorman, A.F.M, Habets, P., Manni, J.J., Drukker, J., 2000. Stapedius muscle fibre composition in the rat. Hear. Res. 141, 169-179.

- Dmitrieva, L.P., Gottlieb, G., 1994. Influence of auditory experience on the development of brain stem auditory evoked potentials in mallard duck embryos and hatchlings. Behav. Neurol. Biol. 61, 19-28.

- Doan, D.E., Igic, P.G., Saunders, J.C., 1996. Middle-ear development VII: umbo velocity in the neonatal rat. J. Acoust. Soc. Am. 99, 1566-1572.

- Doyle, W.J., Webster, D.B., 1991. Neonatal conductive hearing loss does not compromise brainstem auditory function and structure in rhesus monkeys. Hear. Res. 54, 145-151.

- Eggermont, J.J., 1986. Defining and determining sensitive periods. Acta. Otolaryngol. Suppl. 429, 5-9.

- Folsom, R.C., Webster, B.A., Thompson, G., 1983. Auditory brainstem responses in children with early recurrent middle-ear disease. Ann. Otol. Rhinol. Laryngol. 92, 249-253.

- Friauf, E., Lohmann, C., 1999. Development of auditory brainstem circuitry. Activity-dependent and activity-independent processes. Cell Tissue Res. 297, 187-195.

- Geal Dor, M., Freeman, S., Li, G., Sohmer, H., 1993. Development of hearing in neonatal rats: air and bone conducted ABR thresholds. Hear. Res. 69, 236-242.

- Goss Sampson, M.A., Kriss, A., 1991. Effects of pentobarbital and ketamin xylazine anaesthesia on somatosensory, brainstem auditory and peripheral sensory motor responses in the rat. Lab. Anim. 25, 360-366.

- Keilmann, A., 1993. Einfluss einer Schalldeprivation auf die Hörbahnreifung bei der Ratte. Laryngorhinootologie 72, 15-18.

- McFadden, S.L., Walsh, E.J., McGee, J., 1996. Onset and development of auditory brainstem responses in the Mongolian gerbil (Meriones unguiculatus). Hear. Res. 100, 68-79.

- Moore, D.R., 1985. Postnatal development of the mammalian central auditory system and the neural consequences of auditory deprivation. Acta. Otolaryngol. Suppl. 421, 19-30.

- $\quad$ Saunders, J.C., Doan, D.E., Cohen, Y.E., 1993. The contribution of middle-ear sound conduction to auditory development. Comp. Biochem. Physiol. A Physiol. 106, 7-13.

- Shaw, N.A., 1988. The auditory evoked potential in the rat-a review. Prog. Neurobiol. 31, 19-45.

- Sohmer, H., Friedman, I., 1992. Prolonged conductive hearing loss in rat pups causes shorter brainstem transmission time. Hear. Res. 61, 189-196.

- Sohmer, H., Freeman, S., Friedman, I., Lidan, D., 1991. Auditory brainstem response (ABR) latency shifts in animal models of various types of conductive and sensori-neural hearing loss. Acta Otolaryngol. 111, 206-211.

- Stockard, J.E., Stockard, J.J., Westmoreland, B.F., Corfits, J.L., 1979. Brainstem auditory-evoked potentials. Normal variation as a function of stimulus and subject characteristics. Arch. Neurol. 36, 823-831. 
- Walger, M., Laska, M., Schneider, I., Diekmann, H., Wedel, von H., 1993. Maturation of auditory evoked potentials in young Guinea pigs with binaural conductive hearing loss. Eur. Arch. Otorhinolaryngol. 250, 362-365.

- Walsh, R.N., 1981. Effects of environmental complexity and deprivation on brain anatomy and histology: a review. Int. J. Neurosci. 12, 33-51.

- Walsh, E.J., McGee, J., Javel, E., 1986. Development of auditory-evoked potentials in the cat. I. Onset of response and development of sensitivity. J. Acoust. Soc. Am. 79, 712-724.

- Webster, D.B., Webster, M., 1977. Neonatal sound deprivation affects brain stem auditory nuclei. Arch. Otolaryngol. 103, 392398.

- Woolf, N.K., Ryan, A.F., 1988. Contributions of the middle ear to the development of function in the cochlea. Hear. Res. 35 , 131-142. 
- 


\section{Chapter 5}

\section{Stapedius muscle fibre}

characterization in the noise exposed and auditory deprived rat

Patrick F.M. Dammeijer ${ }^{1}$, Paul van Dijk ${ }^{2}$, Michelene N. Chenault ${ }^{3}$, Johannes J. Manni ${ }^{1}$, Henk van Mameren²

1 Department of Otorhinolaryngology and Head and Neck Surgery, University Hospital Maastricht, The Netherlands.

2 Department of Anatomy/Embryology, University of Maastricht, The Netherlands. ${ }^{3}$ Department of Methodology and Statistics, University of Maastricht, The Netherlands.

Published in Hearing Research 2007, 233(1-2), 54-66. 


\begin{abstract}
In skeletal muscle, interventions that unload the muscle cause slow-to-fast myosin heavy chain (MHC) conversions, whereas fast-to-slow conversions are seen when the muscles are engaged in resistance training and endurance exercise. The stapedius muscle (SM) is reported to prevent cochlear damage by noise. This theory may be supported by showing comparable changes of muscle fibre composition when ears are exposed to longstanding noise (SM training). Comparable changes after sound deprivation (SM unloading) would suggest that the SM needs a certain degree of daily activity evoked by environmental sound to sustain its normal composition.

We investigated the difference in myosin composition of SM fibres from rats exposed to noise, from auditory deprived rats and from rats exposed to low level ambient noise (control group). Consecutive complete SM cross-sections were processed by enzymehistochemistry to determine acid/alkali lability of myofibrillar adenosine triphosphatase (mATPase) and by immunohistochemistry using MHC antibodies. Fibres were assigned to mATPase type I, IIA, IIX or 'Miscellaneous' categories. Per mATPase category, the fibres were attributed to groups with specific MHC isoform compositions. Auditory deprivation lasting nine weeks was accomplished by closure of the external meatus at the age of 3 weeks. A slow to fast shift was seen in these rats when compared to the control group.

The noise exposed group was exposed to 65-90 dB sound pressure level during a period lasting nine weeks from the age of 3 weeks onwards. A shift from an overwhelming presence of type mATPase IIX, as seen in the control group, to type mATPase IIA occurred in the noise exposed group. Also, more MHC IIA/IIX hybrid fibres were found in the mATPase IIX category. An adaptive response to the acoustic environment in the characteristics of the fibres of the SM, comparable to the response in skeletal muscles on unloading and training activity, can be ascertained. This supports the theory that the SM plays an active role in modulating external acoustic energy on entry to the cochlea. Our results are also in favour of another postulated function of the SM, the unmasking of high frequency signals in low frequency background noise.
\end{abstract}




\subsection{Introduction}

Contraction of the middle ear muscles changes sound transmission through the middle ear. In this respect the stapedius muscle (SM) is supposed to be the most important in humans (Møller, 1965) as well as in the rat (Berge et al., 1990; Pilz et al., 1997). Acoustic stimulation above about 80 decibel (dB) sound pressure level (SPL) results in a contraction of middle ear muscles of which the intensity correlates with the intensity of noise (Berge et al., 1990; Pilz et al., 1997). Different functions have been attributed to the SM. Several authors have shown that the SM protects the subject from noise induced hearing loss (NIHL) (Borg et al., 1983; Zakrisson, 1975). Moreover the SM can decrease the masking of high-frequency signals by low-frequency noise (i.e., the upward spread of masking) (Borg et al., 1984; Pang and Guinan, 1997) hereby improving human perception of speech in noise (Wormald et al., 1995).

The SM should be able to contract fast and sustain activity for longer periods of time if damage due to sudden and longstanding high-level noise exposure is to be prevented. Knowledge of the myosin composition of the SM fibres will contribute to the understanding of the functional properties of the SM. Investigations involving the SM fibre characteristics have been previously conducted in a variety of species (for review of literature, see Dammeijer et al., 2000).

Skeletal muscle fibres are generally classified as fast glycolytic, fast oxidative, and slow oxidative, which correspond to the speed of shortening, energy metabolism, and fatigue resistance of the muscle fibres. Typically, there are four myosin heavy chain (MHC) isoforms expressed in adult skeletal muscles: one slow type, designated as type I (or cardiac-B) and three fast types, designated as IIA, IIX, and IIB. Other MHCS have been described; super fast IIM, laryngeal IIL and extraocular myosin are expressed in only a few specialized muscles (Wieczorek et al., 1985; Perie et al., 2000).The cardiac- $\alpha$ MHC isoform is expressed in the masseter muscle of the adult rat (Bredman et al., 1992) and the SM of the rat (Dammeijer et al., 2000), a finding that was associated to the branchial arch origin of these muscles. The MHC isoforms each have distinctly different myofibrillar adenosine triphosphatase (mATPase) and shortening velocity properties, which thereby determine the intrinsic functional properties of the individual fibres in which they are expressed. This provides the molecular basis of the functional diversity of a muscle fibre (Pette and Staron, 1990; Schiaffino and Reggiani, 1996; Rivero et al., 1998; Caiozzo et al., 2003).

Despite the differences in architecture of the middle ear of the rat and the middle ear in humans, the biomechanical organisation of the ossicular chain and the middle ear muscles seems to be similar. It justifies comparative studies in this field by using the rat as a model (Berge and Wirtz, 1989). The fast myosin types IIA and IIX, compared to type $\| B_{1}$ are best associated with fatigue resistance, and were found in the majority of the adult rat SM fibres (Dammeijer et al., 2000). It was shown that the majority of SM fibres displayed MHC hybridism at three and six weeks of age (Dammeijer et al., 2006). We found that the decrease of MHC hybridism of SM fibres after the 21st post natal day (PND) was the result of a 
continuing developmental process and suppose that it is the adaptation of the muscle to sound stimuli in the animal facility (Dammeijer et al., 2006).

The present study investigates whether the SM, when rats were subjected to an environment of diminished or enhanced levels of noise, shows changes of muscle fibre characteristics similar to muscle fibre profiles do as reported in skeletal muscles; diminished noise level (SM) comparable to unloaded (skeletal muscles) and enhanced noise level (SM) comparable to trained (skeletal muscles). Skeletal muscles show an adaptation in muscle fibre characteristics in response to decreases in neuromuscular activity. Usually reduced neuromuscular activity causes a shift towards fast MHC isoforms at the expense of the slow MHC I. The magnitude of this shift varies among the different animal and experimental models used (Talmadge, 2000). Skeletal muscles also show a reverse adaptation of muscle fibre characteristics in response to increase in use. In the beginning, adaptation may be only noticed in the level of enzymes involved in pathways of ATP production (Chillibeck et al., 1998; Tikkanen et al., 1995), whereas later an increase in muscle mass may be seen (Chalmers et al., 1992). However, the adaptive response to increased activity also involves a change in muscle fibre type composition, determined either by the mATPase-based classification (Andersen and Henriksson, 1977; Mabuchi et al., 1982) or determined by their MHC isoform expression (Demirel et al., 1999; Roy et al., 1997; Schuler and Pette, 1996). In the rabbit extensor digitorum longus (EDL) adaptation of muscle fibres also includes fibre degeneration and fibre replacement during chronic low frequent stimulation (CLFS) (Maier et al., 1986). The interspecies difference is illustrated by the absence of this fibre replacement by stimulation-induced fibre degeneration during CLFS of the EDL in the rat (Delp and Pette, 1994). In this respect, it seems especially relevant to compare changes which appear during training of the rat SM with changes which appear in trained rat skeletal muscles.

Fibre type conversions in skeletal muscles occur in a graded and orderly sequential manner. The best examples of these conversions are $\mathrm{MHC}$ isoform exchanges. For the $\mathrm{MHC}$, this involves a sequence going from the fastest MHC isoform (MHC IIB) to the slowest (MHC I) isoform, and vice-versa (Pette and Staron, 1997). Especially during the process of adaptation, MHC isoform hybridism is a common finding in muscle fibres (Staron and Pette, 1993). Permanent MHC hybridism of adult muscle fibres facilitates a smooth but possibly relative fast adaptation to changing demands (Caiozzo, 2002). These findings in skeletal muscles may be applied to the SM in order to get better understanding of its function. A significant change in SM MHC isoform expression following noise exposure would support the theory that the major function of the SM is to prevent potentially harmful noise from reaching the cochlea.

We studied the muscle fibre composition of the SM of rats 12 weeks of age, which have been exposed to different noise environments. Three groups were investigated. The first group of rats was kept in quarters with normal ambient noise (measurements showed a level of noise not exceeding $60 \mathrm{~dB}$ SPL in the range 0.1-20 kHz). In the second group of rats bilateral external meatal closure was surgically performed on PND 21, which induced an average of $30 \mathrm{~dB}$ conductive hearing loss (for measurements see Dammeijer 
et al., 2002). The rats in the third group were exposed to a high level of noise starting on PND 21. These rats were exposed to cycles of 1 second narrow band noise at random frequencies (range: $0.1-12 \mathrm{kHz}$ and 65-90 dB SPL alternating with 1 second of silence) (ambient noise level). There were several reasons to choose this kind of noise exposition. First, the intensity of the sound was set at a level that is known to activate the middle ear reflex (MER) effectively. At intensities above $75 \mathrm{~dB}$ for most frequencies, the SM is activated (Berge et al., 1990) and with increasing stimulus intensity the electromyogram amplitude of the SM increases logarithmically (Pilz et al., 1997). Also, chronic exposition to noise above certain intensity is known to cause at least a temporary threshold shift (TS). In chinchillas a 10 days course of exposure to an octave band noise centred at $0.5 \mathrm{kHz}$ at $95 \mathrm{~dB}$ SPL ( $6 \mathrm{~h}$ on/18 h off) provoked a significant TTS (Henselman et al., 1994). Such an exposure, may finally result in a permanent threshold shift (PTS), and is likely to have an effect on the threshold of the MER activation. This could interfere with our intension to load the stapedius muscle within physiological limits. Therefore, our stimulation intensity did not exceed the level of $95 \mathrm{~dB}$ SPL. In an earlier study we showed that the stimulus used in the present study did not cause any cochlear damage in the rat (Dammeijer et al. 2002). Moreover, intermittent noise exposure produces less TS than continuous noise exposure with the same amount of energy (Quaranta et al., 1998). Also, a narrow band noise instead of a pure tone was used to activate the MER more effectively (Pilz et al., 1997). Finally, continuous exposition to a tone is likely to cause an SM reflex decay (Ferraro et al., 1981); varying not only frequency but also the intensity prevents a reflex decay (Borg, 1980).

In the present study the same method of muscle fibre classification was applied as in our earlier research on the SM (Dammeijer et al., 2000, 2006). We hypothesize that the MHC isoform conversions in the rat SM fibres, following sound deprivation or noise exposure are similar to the MHC isoform conversions as described in rat skeletal muscles following inactivity or increased activity respectively.

\subsection{Materials and methods}

\subsubsection{Animals and procedures}

Care for laboratory animals and all animal procedures were in accordance with the Dutch law on animal care. Thirty-seven newborn Norway-Brown rats were raised with their mothers exposed to ambient noise. They were weaned at postnatal day (PND) 21. Then they were randomly assigned to three groups.

One group Norway-Brown male rats $(n=13)$ was raised in normal quarters at ambient noise levels (conducted measurements showed a level of noise not exceeding $60 \mathrm{~dB}$ in the range $0.1-20 \mathrm{kHz}$ ) and will be referred to as the control group. In the auditory deprived group $(n=12)$ a conductive hearing loss was created by surgical closure of the external meatus at PND 21. The animals were anaesthetized using a ketamin (1.3 ml/kg i.m.) and xylazine ( $0.1 \mathrm{ml} / \mathrm{kg}$ i.m.) combination. Both ear canals were obliterated by incising the skin near the tympanic membrane, elevating the meatal skin towards the pinnae, and then 
the skinflaps were approximated using vicryl 5.0 sutures (Ethicon ${ }^{\circledR}$ ). During the experiment the ears were inspected weekly; no signs of infection were noted.

The rats in the noise exposed group $(n=12)$ were kept for a period of 9 weeks in quarters, where they were exposed to cycles of 1 second narrow band noise of at random frequencies (range: $0.1-12 \mathrm{kHz}$ and 65-90 dB SPL alternating with 1 second of silence (ambient noise level).

\subsubsection{Muscle dissection and tissue processing}

Before the removal of the stapedius muscles at PND 84 (12 weeks of age) the animals were anaesthetized using ketamin (1.3mg/kg i.m.) and xylazine $(0.1 \mathrm{ml} / \mathrm{kg}$ i.m.) and for other purposes brainstem auditory evoked responses (ABR) were recorded (Dammeijer et al., 2002). Two EDLs were removed in all groups for reference purpose concerning the histochemical processing procedures.

After electrophysiological recordings (Dammeijer et al., 2002), the rat was decapitated and the mandible was removed to show the bulla (equivalent to the tympanic cavity in humans). The caudal wall of the bulla was opened to approach the pyramidal process (containing the SM belly) and the middle ear ossicles. The lateral bony wall of the pyramidal process, which protrudes in the tympanic cavity, was broken off. SM fibres did not attach to this bony chip. All muscle fibre bundles of the SM were released from the remaining walls of the pyramidal process by blunt dissection. Then, the SM and the stapes were taken out. The stapes was cut off of the SM tendon before stretching the entire SM (mean length 1.2 $\mathrm{mm}$ ) in a drop of Tissue-tek ${ }^{\circledR}$ (O.C.T. compound) poured between two small wooden matches on a slide. Some SM fibres are arranged oblique or more or less perpendicular to the tendon in situ (Berge and Wirtz, 1989). Stretching of the SM prevents longitudinally cutting of these fibres. Then the specimen was frozen in melting isopentane pre-cooled in liquid nitrogen. The muscles were stored in airtight canisters at $-80^{\circ} \mathrm{C}$.

The entire SMs and parts of the EDL were cut to cross-sections $(10 \mu \mathrm{m})$ on a cryostat microtome at $-20^{\circ} \mathrm{C}$. Each SM yielded about 100 cross-sections which were numbered from the origin to the tendon. Consecutive cross-sections of the EDL parts were numbered also. One SM and one EDL cross-section were mounted on the same slide. The SM cross-sections numbered 20 up to 60 and accompanying EDL cross-sections were processed by enzymehistochemistry $(E H C)$ or immunohistochemistry $(\mathrm{IHC})$ and consequently were examined with a microscope. Two levels per SM were chosen for fibre type characterization, near SM cross-section numbers 30 and 50. The cross-sections of these levels were photographed. A level contains ten consecutive EHC or IHC processed cross-sections. From the auditory deprivation group, the noise exposed group and the control group, respectively 12, 7 and 6 levels could be used, with each level containing 100 fibres. It was not possible to cut at two levels in each muscle, resulting in 6, 3 and 3 usable muscles from 6,3 and 2 rats from, respectively, the auditory deprivation group, the noise exposed group and the control group. 


\subsection{3 mATPase characterization}

A modification of the procedure of Brooke and Kaiser (1970) was carried out for the acid preincubation method (Ogilvie and Feeback, 1990). Type I fibres stained dark, type IIA fibres stained light, type IIX and IIB fibres presented intermediate staining. The mATPase alkali lability protocol was adapted from Guth and Samaha (1970). The sections were fixed in buffered 2\% methanol-free paraformaldehyde and stained after pre-incubation at pH 10.4. The double pre-incubation method according to Sant'Ana Pereira et al. (1995a) was used to determine fibres which contain myosin type IIX.

Fibres were assigned to different mATPase categories based on combinations of staining intensities (Table 5.1). Myofibrillar ATPase profiles were defined according to Gorza (1990), supplemented with Sant'Ana Pereira's et al. criteria for myosin type IIX (1995a). Fibres that did not meet the criteria of the mATPase categories type I, IIA, IIX or IIB were assigned to one of the types Miscellaneous ('Misc').

Table 5.1 Combinations of fibre staining on mATPase activity after different pre-incubation based on Gorza (1990) and Sant'Ana Pereira et al. (1995a).

\begin{tabular}{llll}
\hline mATPase category & $\mathrm{pH} 4.35$ & $\mathrm{pH} 10.4$ & double pre-incubation \\
\hline type I & ++ (dark) & - (light) & - (light) \\
type IIA & - (light) & ++ (dark) & - (light) \\
type IIX & + (intermediate) & $++($ dark $)$ & + +++ (intermediate/dark) \\
type IIB & + (intermediate) & + (intermediate) & - (light) \\
type 'Misc' a & mATPase activity profiles other than above & \\
\hline
\end{tabular}

${ }^{a}$ three 'Misc' categories were discerned in this study; 'Misc' $1,++,+,-$, 'Misc' $2,-,++,+$, 'Misc' $3,+,+,-$.

\subsubsection{MHC isoform characterization}

Each fibre categorized along the criteria given in Table 5.1 was further characterized by immunoreactivity (IR), negative (-) and positive (two levels, + or ++ ), on a panel of MHC antibodies (mAbs), respectively specific to MHC I (219-1D1) (Bredman et al., 1992; Sant'Ana Pereira et al., 1995b), MHC IIA (333-7H1) (Bredman et al., 1992; Sant'Ana Pereira et al., 1995b), MHC IIX (6H1) (Lucas et al., 2000), MHC IIB (MHC BF-F3) (Schiaffino et al., 1989) and to MHC cardiac- $\alpha$ (249-5A4) (Bredman et al., 1992). The indirect unconjugated technique after Moorman et al. (1984) was applied. EDL cross-sections on the same slides as well as sections without primary antibody incubation were used to determine correctness of processing the slides.

\subsubsection{Selection of muscle fibres}

On average 200 fibres were present in each cross-section of the entire muscle. None of the cross-sections processed, by either EHC or IHC, showed selectively located muscle fibre staining, which could be indicative for unequal distribution of muscle fibre types. An area that only contained crosssectioned fibres was selected on the photograph of the cross-section processed for EHC after pre- 
incubation at pH 4.35. Then 100 adjacent fibres were numbered in this area. These fibres were identified on the other cross-sections of the given level and numbered correspondingly. A fibre, which could not be matched to one of the cross-sections of the level examined was removed from the selection and replaced by an adjacent fibre that showed a staining intensity after pre-incubation at pH 4.35 equal to the excluded fibre. Replacement occurred for about 0-2 fibres per level examined.

Two of the authors independently scored the staining intensity of the selected fibres,-+ or ++ . Scoring was carried out per cross-section. Per level, the score of both examiners for maximal four fibres was different. Assignment of these fibres was established by re-evaluation of the cross-sections in mutual agreement by the two examiners. For illustration, the scoring of 30 SM fibres, 10 from the auditory deprived, noise exposed and the control group each, being,,-+ or ++ is given in Table 5.2 on the basis of the staining qualities on the photographs in Figs. 5.1a, 5.1b and 5.1c respectively. On the basis of staining intensity of three mATPase activity assays, a fibre was assigned to a certain mATPase category. The MHC isoform contents were further determined by means of the IR strength (negative (-), weak (+) and strong $(++)$ of the specific mAbs in each identified fibre. In the Figs. 5.1a, 5.1b and 5.1c, the mATPase staining after pre-incubation at pH 10.4 is not shown because these photographs do not display enough details for illustration purposes. The photographs in the Figs. 5.1a, 5.1b and 5.1c are arranged in order by the rising numbers of the cross-sections as they were cut from the SM. This illustrates best the technique of tracking down each single identified fibre throughout consecutive cross-sections of the SM, because then the differences between the boundaries of the muscle fibres and the position of the fibres in the consecutive cross-sections are the smallest.

Fig. 5.1a. SM serial consecutive cross-sections from one level of an SM in the auditory deprived group.

Ten fibres numbered (1-10) are identified on each consecutive cross-section. The boundaries of the fibres are designated by dotted lines. The photographs in this figure represent the cross-sections which are processed for mATPase activity after pre-incubation pH 4.35 or the double pre-incubation or for MHC characterization by the mAbs to MHCI, IIA, IIB or IIX. Cross-sections processed for mATPase activity after pre-incubation $\mathrm{pH}$ 10.4 and for MHCcharacterization by the $\mathrm{AAb}$ to MHCcardiac- $\alpha$ arenotshown. Numbers in the right upper corner of the photographs correspond to the cross-section number of the level being presented. Staining intensities of the fibres are given in Table 5.2.

Fig. 5.16. SM serial consecutive cross-sections from one level of an SM in the noise exposed group. Ten fibres numbered (11-20) are identified on each consecutive cross-section. The same histochemistry and immunoreactivity panel is shown as in Fig. 5.1a. Numbers in the right upper corner of the photographs correspond to the cross-section number of the level being presented. Staining intensities of the fibres are given in Table 5.2.

Fig. 5.1c. SM serial consecutive cross-sections from one level of an SM in the control group. Ten fibres numbered (21-30) are identified on each consecutive cross-section. The same histochemistry and immunoreactivity panel is shown as in Fig. 5.1a. Numbers in the right upper corner of the photographs correspond to the cross-section number of the level presented. Staining intensities of the fibres are given in Table 5.2. 


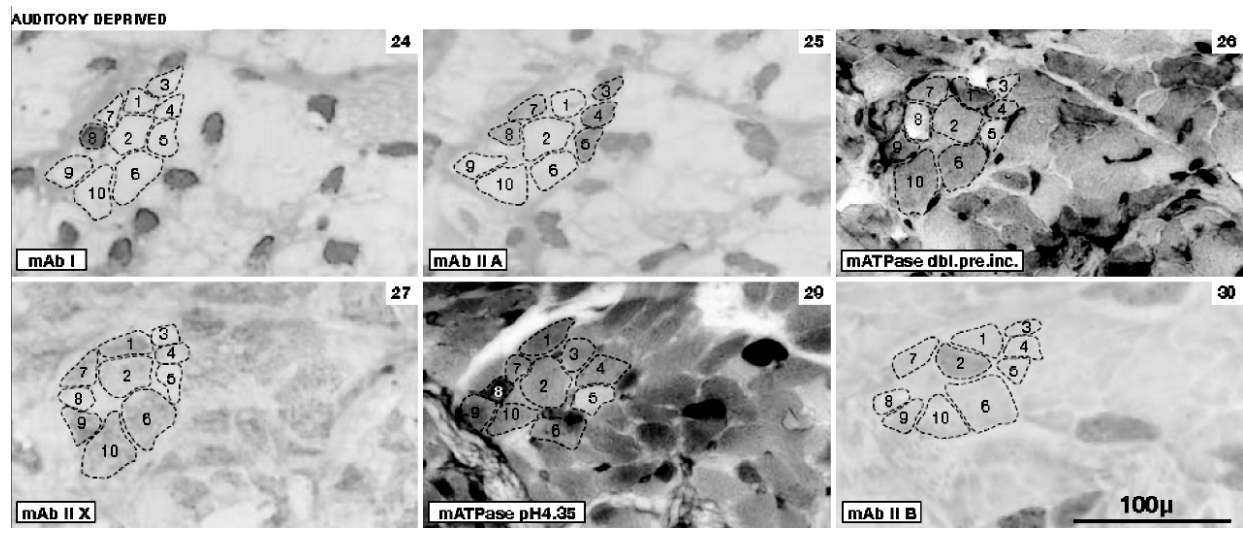

Fig. $5.1 a$

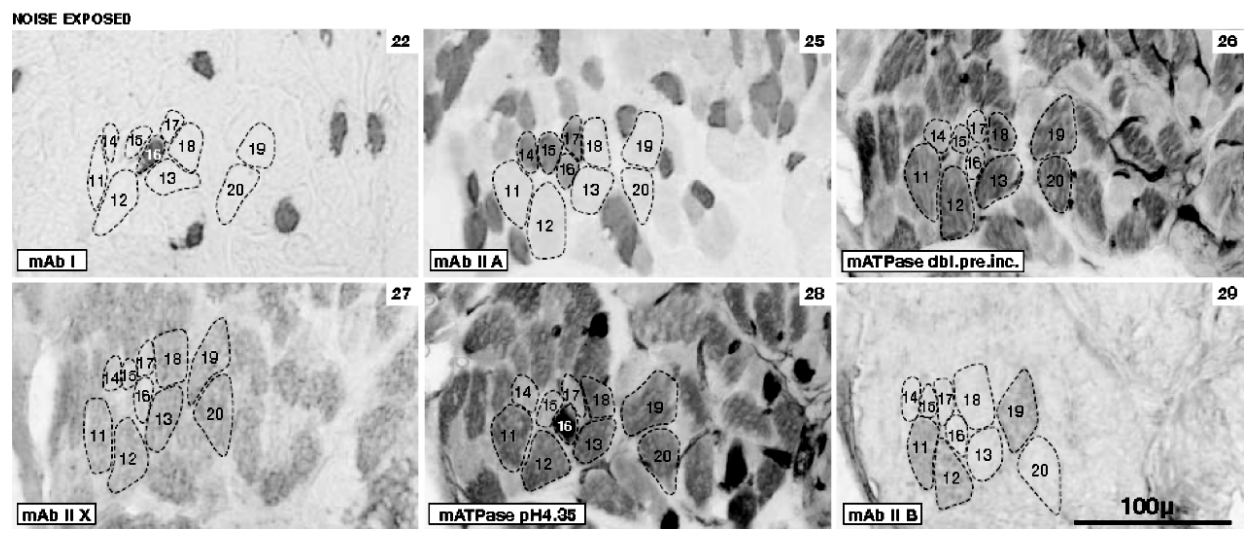

Fig. 5.16

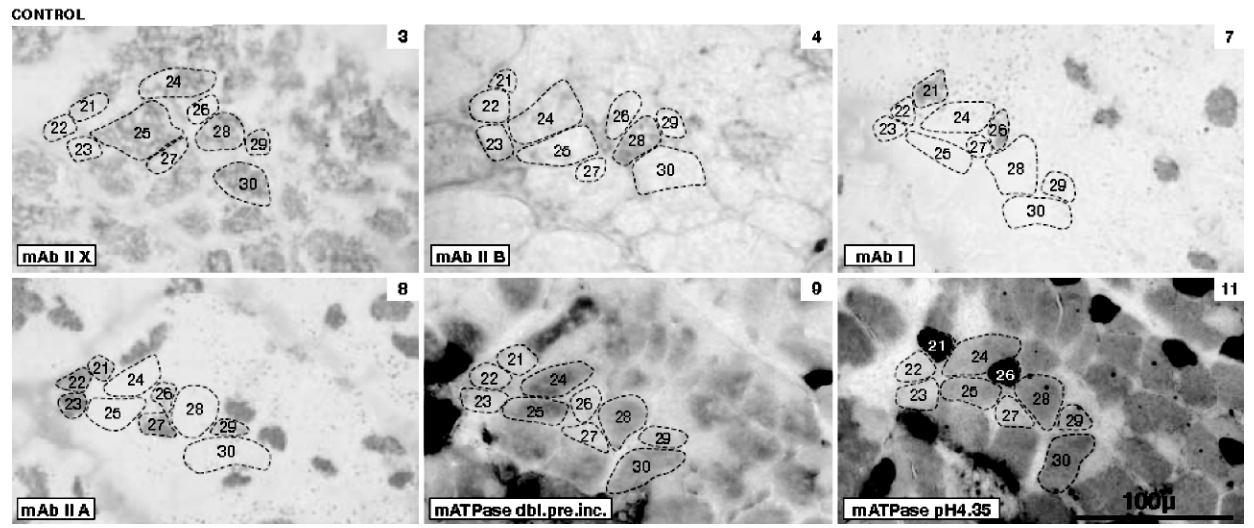

Fig. 5.1C 
Table 5.2 Staining combinations of 30 SM fibres; 10 of one SM from each experimental group, deducted from the staining intensities on the photographs in Figs. 5.1a-c

\begin{tabular}{|c|c|c|c|c|c|c|c|c|}
\hline Fibre & pH 4.35 & $\mathrm{pH} 10.4^{\#}$ & dbl. pre-inc. & mATPase Cat. & mAb I & $m A b \| A$ & $m A b \| B$ & $m A b \| X$ \\
\hline \multicolumn{9}{|c|}{ Auditory deprived } \\
\hline 1 & + & ++ & ++ & IIX & - & - & - & ++ \\
\hline 2 & + & ++ & + & IIX & - & - & + & + \\
\hline 3 & + & ++ & - & $\| A$ & - & ++ & - & - \\
\hline 4 & + & ++ & + & 'Misc' 2 & - & ++ & - & + \\
\hline 5 & - & ++ & - & $\| A$ & - & ++ & - & - \\
\hline 6 & + & ++ & ++ & IIX & - & - & - & ++ \\
\hline 7 & + & ++ & + & IIX & - & + & - & ++ \\
\hline 8 & ++ & + & - & 'Misc' 1 & ++ & + & - & - \\
\hline 9 & + & ++ & ++ & IIX & - & - & - & ++ \\
\hline 10 & + & ++ & ++ & IIX & - & - & - & ++ \\
\hline \multicolumn{9}{|c|}{ Noise exposed } \\
\hline 11 & + & ++ & ++ & IIX & - & - & + & ++ \\
\hline 12 & + & ++ & ++ & IIX & - & - & + & ++ \\
\hline 13 & + & ++ & ++ & IIX & - & - & - & ++ \\
\hline 14 & - & ++ & - & $\| A$ & - & ++ & - & - \\
\hline 15 & - & ++ & - & $\| A$ & - & ++ & - & - \\
\hline 16 & ++ & + & - & 'Misc' 1 & ++ & + & - & - \\
\hline 17 & - & ++ & - & $\| A$ & - & ++ & - & - \\
\hline 18 & + & ++ & ++ & IIX & - & - & - & ++ \\
\hline 19 & + & ++ & ++ & IIX & - & - & + & ++ \\
\hline 20 & + & ++ & ++ & IIX & - & - & - & ++ \\
\hline \multicolumn{9}{|c|}{ Control } \\
\hline 21 & ++ & + & - & 'Misc' 1 & ++ & + & - & - \\
\hline 22 & - & ++ & - & $\| A$ & - & ++ & - & - \\
\hline 23 & - & ++ & - & $\| A$ & - & ++ & - & - \\
\hline 24 & + & ++ & ++ & IIX & - & - & - & ++ \\
\hline 25 & + & ++ & ++ & IIX & - & - & - & ++ \\
\hline 26 & ++ & + & - & 'Misc' 1 & ++ & + & - & - \\
\hline 27 & - & ++ & - & $\| A$ & - & ++ & - & - \\
\hline 28 & + & ++ & ++ & IIX & - & - & + & ++ \\
\hline 29 & + & ++ & + & IIX & - & + & - & ++ \\
\hline 30 & + & ++ & ++ & IIX & - & - & - & ++ \\
\hline
\end{tabular}


Legend Table 5.2

The mATPase activity staining intensity after pre-incubation $\mathrm{pH} 4.35, \mathrm{pH} 10.4^{*}$ and double pre-incubation, is shown as staining light $(-)$, intermediate (+) or dark (++). The IR to four mAbs against MHC I, MHC IIA, MHC IIB and MHC IIX is shown as negative (-), weak (+) or strong (++), which correlates with the relative amount of MHC isoform expressed. Each fibre is assigned to the mATPase category according to the mATPase activity profile (see Table 5.1). The mATPase activity profiles of the categories 'Misc' (staining after preincubation pH 4.35, 10.4 and double pre-incubation) are 'Misc' $1++,+,-$ and 'Misc' $2-,++,+$. Auditory deprivation, noise exposed and control (ambient sound) depict the group of where the numbered fibres originate from. ${ }^{*}$ The photographs of the mATPase activity staining after pre-incubation pH 10.4 are not shown in Figs. 5.1a-c.

\subsubsection{Statistical analysis}

Chi-square analysis (SPSS11.5) was performed to test homogeneity of classification within each of the three experimental groups (noise exposed, auditory deprived and control) and thereafter to investigate whether there was a relation between classification and group.

\section{$5.3 \quad$ Results}

\subsubsection{Similarity of fibre composition in SMs of the same experimental group compared to differences between groups}

A Chi-square test was performed first for each of the three experimental groups separately to investigate the similarity of the SMs of rats within a group relative to mATPase type classification of the SM fibres. Assignment of fibres to the mATPase type 'Misc' 3 category occurred only in very small numbers in the auditory deprived group. These fibres were not identified in the noise exposed and control groups. Furthermore, mATPase type I classification was almost negligible in the auditory deprived group. Therefore this classification was not included when testing for similarity within this group. The Chi-square statistics were all above 0.9 , indicating that mATPase classification distribution did not differ significantly between SMs within a group.

Because the fibre counts in the mATPase type I category were almost negligible for the auditory deprived group, it seemed appropriate to compare the mATPase categorization for three groups pair-wise. Chisquare analysis resulted in a significance level of less than $0.05(p<0.0005)$ when comparing the numbers of fibres in mATPase categories from the auditory deprived group to either the noise exposed group or the control group. When the auditory deprived group was compared to either the noise exposed or control group it showed relatively less assignment of fibres to the mATPase types type I and IIA categories, while the auditory deprived group had relatively more type 'Misc' 2 fibres than the noise exposed group. When the noise exposed group was compared to the control group a $p$-value of 0.001 was obtained, as a result of significant differences between these groups expressed in the existence of relatively less mATPase type I fibres and relatively more mATPase type IIA fibres in the noise exposed group. 


\subsubsection{Fibres with dominant expression of the $\mathrm{MHCl}$ isoform}

All SM fibres which showed strong (++) IR to the mAb to MHC I also showed dark (++) mATPase staining after pre-incubation at pH 4.35 and were assigned to the mATPase category type I or type 'Misc' 1 (see Table 5.3). The number of fibres showing this strong (++) IR to the $\mathrm{mAb}$ to $\mathrm{MHCI}$ in the auditory deprived group was 10.5\%, in the noise exposed group 14.3\% and in the control group 17.7\%. The fraction of all SM fibres that could be assigned to mATPase category type I according to the criteria mentioned in Table 5.1 in the auditory deprived group was 0.2\%, in the noise exposed group 3.7\% and the control group 7.7\%. Only a few of these fibres co-expressed cardiac- $\alpha$ MHC. In all three groups about $10 \%$ of the SM fibres displayed strong (++) IR to the mAb to MHC I and weak (+) IR to the mAb to MHC IIA. These fibres showed an intermediate mATPase staining after pre-incubation at $\mathrm{pH} 10.4$, and were therefore assigned to the mATPase category type 'Misc' 1, in stead of mATPase type I. Co-expression of the MHC I, MHC IIA and cardiac- $\alpha$ MHC was seen in nearly half of the type 'Misc' 1 fibres in the SMs of the noise exposed group, more than in the control group or in the auditory deprived group (4.7\% versus $0.7 \%$ and $2.8 \%$ respectively).

The number of fibres expressing cardiac- $\alpha \mathrm{MHC}$ was more prevalent in the noise exposed group than in the control or, auditory deprived group; percentages were $6 \%, 1.9 \%$ and $2.9 \%$ respectively.

\subsection{3 mATPase type IIA fibres and expression of the MHC IIA isoform}

The number of fibres assigned to the mATPase type IIA category was 13.6\% in the auditory deprived group, and 30\% in the noise exposed group and 23\% in the control group (see Table 5.3 and Fig. 5.2). This is a statistically significant difference ( $p$-values $<0.0005)$. All these fibres showed $(++) I R$ to the mAb to MHC IIA. Besides a very few fibres in the auditory deprived group all fibres in this category were pure MHC IIA. The average cross-sectional area (CSA) of the fibres assigned to MATPase type IIA and category type 'Misc' 2 was almost similar. The fibres assigned to the mATPase category type 'Misc' 2 due to the intermediate mATPase staining after double pre-incubation, also showed (++) IR to the mAb to MHC IIA. They all co-expressed MHC IIX, which was confirmed by the (+) IR to the mAb to MHC IIX. The percentage of fibres assigned to this category was 6.5\%, 3.3\% and 5.7\% for the auditory deprived, noise exposed and the control group respectively.

Only in the auditory deprived group very few fibres (0.3\%) were assigned to the mATPase category type 'Misc' 3; these fibres expressed MHCI as well as MHC IIA. Overall MHC IIA expression, determined by IR to the mAb to MHC IIA, differed significantly; MHC IIA was found in 52.2\% of the fibres in the noise exposed group, $40.2 \%$ in the control and $33.2 \%$ in the auditory deprived group ( $p$-values $<0.0005$ ).

\subsection{4 mATPase type IIX fibres and expression of the MHC IIX isoform}

The majority of SM fibres were assigned to the mATPase type IIX category in all three groups: 69.2\% for the auditory deprived, 52.4\% for the noise exposed, and 53.7\% for the control groups (see Table 5.3 and Fig. 5.2). Approximately 10\% of these fibres were MHC hybrids except for the control group, where nearly all the mATPase type IIX category fibres were pure MHC IIX. Co-expression with 
Table 5.3 SM fibres in the experimental groups (auditory deprived, noise exposed and control) assigned to mATPase categories type I, type IIA, type IIX and 3 type 'Misc' categories and their MHC expression.

\begin{tabular}{|c|c|c|c|c|c|c|c|c|}
\hline \multirow{2}{*}{$\begin{array}{l}\text { mATPase } \\
\text { category }\end{array}$} & \multicolumn{5}{|c|}{$\mathrm{mAb} I \mathrm{R}$} & \multicolumn{3}{|c|}{ Mean $\%$ of fibres in the groups with different noise exposure } \\
\hline & $\alpha$ & I & $\| A$ & IIB & IIX & auditory deprived & noise exposed & Control \\
\hline \multirow[t]{3}{*}{ type I } & & & & & & $(0.2)$ & $(3.7)$ & $(7.7)$ \\
\hline & - & ++ & - & - & - & 0.1 & 2.4 & 6.5 \\
\hline & + & ++ & - & - & - & 0.1 & 1.3 & 1.2 \\
\hline \multirow[t]{3}{*}{ type 'Misc' 1} & & & & & & $(10.3)$ & $(10.6)$ & $(10)$ \\
\hline & - & ++ & + & - & - & 7.5 & 5.9 & 9.3 \\
\hline & + & ++ & + & - & - & 2.8 & 4.7 & 0.7 \\
\hline \multirow[t]{3}{*}{ type IIA } & & & & & & $(13.6)$ & (30) & (23) \\
\hline & - & - & ++ & - & - & 13.3 & 30 & 23 \\
\hline & - & + & ++ & & - & 0.3 & & \\
\hline \multirow[t]{2}{*}{ type 'Misc' 2} & & & & & & (6.5) & (3.3) & $(5.7)$ \\
\hline & - & - & ++ & - & + & 6.5 & 3.3 & 5.7 \\
\hline \multirow[t]{3}{*}{ type 'Misc' 3} & & & & & & $(0.3)$ & (0) & (0) \\
\hline & - & + & + & - & - & 0.2 & & \\
\hline & - & + & ++ & - & - & 0.1 & & \\
\hline \multirow[t]{8}{*}{ type IIX } & & & & & & (69.2) & (52.4) & $(53.7)$ \\
\hline & - & - & - & - & ++ & 60.3 & 42.3 & 49.2 \\
\hline & - & - & - & + & + & 0.3 & 0.1 & 1 \\
\hline & - & - & - & + & ++ & 6.1 & 1.7 & 1.7 \\
\hline & - & - & - & ++ & + & & & 0.3 \\
\hline & - & - & + & + & ++ & 0.3 & 0.3 & \\
\hline & - & - & + & - & + & 0.2 & 2.1 & 0.5 \\
\hline & - & - & + & - & ++ & 2 & 5.9 & 1 \\
\hline
\end{tabular}

Column 1 of the table shows the mATPase categories found. The mATPase activity profiles of the categories 'Misc' (staining after pre-incubation pH 4.35, 10.4 and double pre-incubation) are 'Misc' $1++,+,-$, 'Misc' 2 -, ++, + and 'Misc' $3+,+,-$. The fibres are subdivided to MHC combination groups based on IR to mAbs against, MHC cardiac- $\alpha$ $(\alpha), \mathrm{MHC}$, MHC IIA, MHC IIB and MHC IIX. IR to the mAbs used is shown as negative (-), weak (+) and strong (++) (See Fig. 5.1 and Tables 5.1 and 5.2). The columns auditory deprivation, noise exposed and control (ambient sound) represent the group from which the muscles originate. The mean percentages of SM fibres assigned to the mATPase based categories are shown in brackets and are subsequently assigned to a mAb IR combination subgroup in that particular mATPase category. 


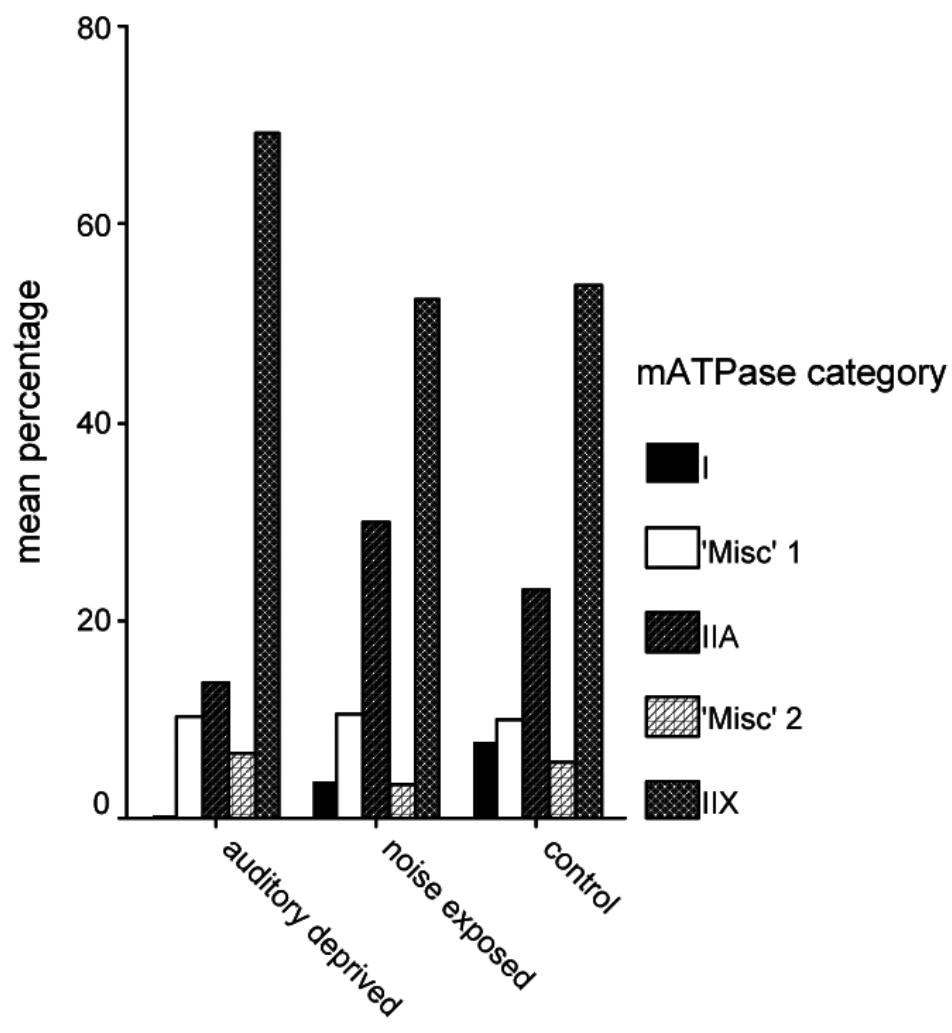

Fig.5.2. Mean percentages of the SM fibres assigned to mATPase categories (mATPase staining following pre-incubation at pH 4.35, pH 10.4 and double pre-incubation) in the three experimental groups (auditory deprived, noise exposed and control). The mATPase type 'Misc'3 category is not included because of scarce occurrence.

MHC IIB in this mATPase category was seen most often in the auditory deprived group (6.7\%). Fibres in the mATPase type IIX category which co-expressed MHC IIA were relatively more frequent in the noise exposed group (8.3\%). Very few fibres show co-expression of MHC IIX, MHC IB and MHC IIA. MHC IIX expression determined by IR to the $\mathrm{mAb}$ to MHC IIX was noticed in $75.7 \%$ of the fibres in the auditory deprived group, $59.3 \%$ and $55.7 \%$ in the control and noise exposed group respectively. A Chi-square analysis of the three groups, with respect to MHC subgroups in the MATPase IIX category with and without a co-expression of MHC IIA with MHC IIX, showed that there were significantly more fibres with both $\mathrm{MHC}$ isoforms in the noise exposed group than in either the control or the auditory deprived group ( $p$-values $<0.0005$ ). No difference was found between the auditory deprived and the control group (sig. $0.765)$. 


\subsubsection{The absence of fibres in the mATPase type IIB category}

No fibres were assigned to the mATPase category type IIB (see Table 5.3 and Fig. 5.2). MHC IIB expression was only seen in the mATPase type IIX category fibres. MHC IIB expression was observed in the auditory deprived, the control and noise exposed group in 6.7\%, 3\% and $2.1 \%$ of the muscle fibres respectively.

\subsubsection{SMs only processed for mATPase activity staining after pre-incubation at pH 4.35}

Due to technical problems with parts of the mATPase method, and because not all mAbs were available during that phase of our research many SMs were only examined with the mATPase method after pre-incubation at pH 4.35. In order to acquire more data concerning changes in fibre type composition, we included SMs only processed for mATPase activity staining after pre-incubation at pH 4.35 of the control, auditory deprived and noise exposed group in this report. In these SMs we were able to evaluate mATPase staining of fibres after pre-incubation at $\mathrm{pH} 4.35$ in complete muscle cross-sections. In 3 SMs from 3 rats of the noise exposed group, all fibres in 8 muscle cross-sections were addressed. A total of 1867 fibres were counted. In 3 SMs from 3 rats of the auditory deprived group, all fibres in 11 muscle cross-sections were addressed, with a total count of 3067 fibres. In 3 SMs from 3 rats of the control group, all fibres in 9 muscle cross-sections were addressed, with a total count of 2178 fibres. The mATPase activity after pre-incubation pH 4.35 categorizes the fibres in the categories dark, light and intermediate. A Chi-square test was performed first for each of the three groups (noise exposed, auditory deprived and control) separately to make sure that the rats within a group were similar relative to the three classifications dark, intermediate and light. When comparing the three groups with respect to mATPase staining intensity of their fibres after pre-incubation at pH 4.35, a significantly different fibre composition is found ( $p$-value $<0.0005$ ). The fibre composition of the auditory deprived group is distinctly different from the other groups with its fibres categorized into intermediate staining relatively much more frequently ( $p$-value $<0.0005$ ). When comparing the noise-exposed with the control group, the noise exposed group had relatively less fibres in the intermediate staining category and relatively more in the light staining category. This difference is also statistically significant ( $p$-value $=0.02$ ). The percentages of SM fibres of the above-mentioned rats are shown in Table 5.4.

Fibres of SMs which are classified in Table 5.3 were also classified just like the nine above-mentioned SMs were and also these data are presented in Table 5.4. The dark staining fibres in Table 5.3 are the fibres in the mATPase type I and type 'Misc' 1 categories, the light staining fibres are the fibres in the mATPase type IIA and type 'Misc' 2 categories and the intermediate staining fibres are the fibres in the mATPase IIX category. 
Table 5.4 Percentages of SM fibres from rats in the experimental groups which are categorized depending on the mATPase staining after pre-incubation at $\mathrm{pH} 4.35$ only.

\begin{tabular}{llll}
\hline & \multicolumn{2}{l}{ mATPase activity after pre-incubation at $\mathrm{pH} 4.35$} & \\
\cline { 2 - 4 } & Dark & Light & Intermediate \\
\hline noise exposed & $17 \%(14.3 \%)$ & $29.7 \%(33.3 \%)$ & $53.3 \%(52.4 \%)$ \\
auditory deprived & $13.1 \%(10.5 \%)$ & $20 \%(20.1 \%)$ & $66.9 \%(69.4 \%)$ \\
Control & $16.3 \%(17.7 \%)$ & $26.2 \%(28.7 \%)$ & $57.5 \%(53.7 \%)$ \\
\hline
\end{tabular}

Between brackets: percentages extracted from Table 5.3 (SM fibres from muscles which are examined on cross-sections with the complete enzymehistochemistry panel).

\subsection{Discussion}

\subsubsection{SM fibre's mATPase category assignment is able to predict its MHC isoform expression to a large extent}

In the present study six different mATPase based fibre types were discerned. The muscle fibre classification system which we present here shows a clear relationship between the mATPase activity after three different pre-incubations of a single fibre and its MHC isoform contents, on a fibre to fibre basis. The findings of Sant'Ana Pereira on skeletal muscles show that the most dominantly expressed MHC isoform largely determines to which mATPase category a fibre is assigned to (Sant'Ana Pereira et al., 1995b). This also applies to the SM. There seem to be only two exceptions to this and these require an explanation. One is the expression of cardiac- $\alpha \mathrm{MHC}$ which always occurs with strong IR to $\mathrm{MHC} \mathrm{I}$. An explanation for this particular co-expression of cardiac- $\alpha \mathrm{MHC}$ and $\mathrm{MHC}$ I may be that their $\mathrm{MHC}$ genes are identified in tightly linked clusters on the same chromosome, another chromosome than the other skeletal MHC genes (Weiss et al., 1999). However its presence does not determine the assignment to either type mATPase I or the type 'Misc' 1. It seems that the presence of cardiac- $\alpha$ MHC does not influence the staining of the mATPase assays applied. The other exception concerns the, though small in number, mATPase 'Misc' 3 category fibres which show a mATPase staining profile that resembles the profile of mATPase type IIB fibres. These 'Misc' 3 category fibres almost have the CSA of a typical mATPase type I fibre and the IHC subclassification shows MHC I and MHC IIA co-expression which makes it a type mATPase 'Misc' category prospectively. The combination of MHC I and MHC IIA in a fibre could have lead to assignment to the mATPase 'Misc' 1 category, but the dominance of MHC IIA probably caused the light mATPase staining after acid pre-incubation, which has resulted in assigning these fibres to an additional mATPase category type 'Misc' 3.

Further examination of the mATPase IIX category fibres by means of IHC reveals that the vast majority of these fibres are 'pure' MHC IIX, but a portion of the fibres shows an expression of MHC IIX with MHC IIB and/or MHC IIA. This could not have been recognized by the use of only the mATPase staining profile. For 
the average mATPase type IIX SM fibre, one can predict fairly the MHC content. For about 10\% of these fibres, a complementary IHC characterization system is needed to determine the exact MHC isoform contents, although in the majority of these MHC hybrid fibres the IR was strong for MHC IIX.

The use of only IHC on serial cross-sections would not have disclosed the dominant $\mathrm{MHC}(\mathrm{s})$ expressed on a fibre to fibre basis. Complementary IHC with EHC characterization provides the necessary refinement of SM fibre classification which neither of the techniques could have provided on its own.

\subsubsection{Results obtained from SMs only processed for mATPase activity staining after pre-incubation at $\mathrm{pH} 4.35$ support the differences found between SMs which were subject for complete analysis}

Intra-muscle, intra-rat and intra-group variation are found to be small in the SMs processed only for mATPase activity staining after pre-incubation at pH 4.35, the variations are of same size in the SMs used for the complete analysis of the experimental groups. This was a convenient finding, because, due to technical difficulties concerning dissecting the SM out of its bony cover, cutting the SM at right angles, performing $\mathrm{IHC}$ and $\mathrm{EHC}$ procedures on consecutive cross-sections, many SMs were not suitable for complete data collection. If we only pay attention to the mATPase activity after pre-incubation $\mathrm{pH}$ 4.35 in Table 5.3, we can discern 3 groups of SM fibres with a mATPase activity that correlates with the overwhelming presence of either MHC I, MHC IIA or MHC IIX/B. These are the fibres which stain dark, light and intermediate respectively after pre-incubation $\mathrm{pH}$ 4.35. The 3 groups of fibres from Table 5.4 are in conformity with, respectively, the sum of the fibres in the mATPase type I and type 'Misc' 1 categories, with the sum of the fibres in the MATPase type IIA and type 'Misc' 2 categories and the number of fibres in the MATPase IIX category from Table 5.3. In the auditory deprived group the muscle fibres were assigned to the group of intermediate staining fibres (MHC IIX dominance) relatively much more frequently ( $p$-value < 0.0005). Comparing the noise exposed group with the control group on the basis of only examination with the mATPase method after pre-incubation at $\mathrm{pH} 4.35$, the noise exposed group had relatively less intermediate staining fibres and relatively more light staining fibres (MHC IIA dominance) ( $p$-value $=0.02$ ). Comparing the percentages of fibres with mATPase staining after preincubation at pH 4.35 only, with the corresponding data extracted from Table 5.3, minimal differences are shown, which strengthens the conclusions drawn from the data presented in Table 5.3.

\subsubsection{SM fibres in the mATPase types 'Misc' are MHC isoform hybrids, and constitute the continuum of fibre types intermediate to the fibre types mATPase I, IIA and IIX}

The mATPase category assignment of the SM fibres strongly correlates with CSA expressed.

Fibres in the mATPase type I category were the smallest and mATPase IIX fibres were the largest. Intermediate in size, with some overlapping, were in rank order from small to large, mATPase type 'Misc' 1, type 'Misc' 3 category, mATPase type IIA and type 'Misc' 2 fibres. All the 'Misc' type categories contain $\mathrm{MHC}$ hybrid fibres only. 
Similar muscle fibre research on serial cross-sections combining IHC with EHC conducted in the rat medial gastrocnemius muscle by Rivero et al. has shown a correlation between 'pure' MHC fibres and fibre CSA. This group described a similar correlation with succinate dehydrogenase (SDH), and $\alpha$-glycerophosphate dehydrogenase (GPD) content. Moreover MHC hybrid fibres, co-expressing two MHC isoforms, generally showed mean CSA, SDH and GPD values lying between their respective pure MHC fibre types (Rivero et al., 1998). Similar to findings in other skeletal muscles (Caiozzo et al., 2003) we assume that a continuum of muscle fibre properties, such as maximum shortening velocity and fatigue resistance is also displayed in the SM MHC 'hybrid' fibres; their properties being intermediate to that of their 'pure' MHC counterparts. These hybrid fibres enable the SM to fine-tune its efficiency for the wide range of forces, velocities, levels of endurance and levels of resistance to fatigue as has been suggested for skeletal muscles (Pette, 2002; Quiroz-Rothe and Rivero, 2004; Stephenson, 2001).

\subsubsection{The fibres of auditory deprived SMs differ significantly from the SMs in the control group with respect to MATPase as well MHC typing}

The mATPase type I fibres apparently are absent in the auditory deprived rats, which complies with the anticipated slow to fast shift of skeletal muscle fibres. The ratio differences of the numbers of type mATPase IIA and type mATPase IIX fibres in the auditory deprived and control group seem to comply with the hypothesized slow to fast shift as well. The auditory deprived SMs show larger numbers of the faster mATPase IIX at the expense of type mATPase IIA fibres.

In this study the rats were included in the experimental groups at the age of 3 weeks. Although the SM fibres are of an adult like functional type, the fibre composition using mATPase and MHC typing differs from that of the situation at the age of 12 weeks. At three weeks of age the majority of SM fibres were assigned to the mATPase type I, type 'Misc' 1, type IIA, type 'Misc' 2 and type IIX categories, with a distribution ratio of $2.5 \%, 10.3 \%, 13 \%, 10 \%$ and $61.5 \%$ respectively (Dammeijer et al., 2006). These findings are similar to those found in the auditory deprived group at 12 weeks in this study. By depriving rats from auditory input from the age of 3 weeks onwards, it appears that the fibre type composition is stagnated in the IIX preponderance situation existing at 3 weeks up to the termination of the experiment at 12 weeks of age. Also the co-expression of MHC IIB with MHC IIX in the mATPase type IIX fibres at three weeks of age, as was described earlier, remained present in the auditory deprived group. Compared to the $54.9 \%$ of SM fibres that were MHC hybrid at the age of 3 weeks the smaller (26.3\%) number of MHC hybrid fibres at 12 weeks suggest a steady state situation of MHC composition adapted to auditory deprivation. The auditory deprivation has directed the MHC outgrowth of the SM fibres in domination of the pure fast fibre type similar to an unloaded muscle.

Within the scope of hypotheses concerning the function of the SM it is stated that the clear effect of external meatal closure (auditory deprivation) on the SM fibre composition in a direction of an unloaded muscle makes it unlikely that a significant activation of the SM occurs as part of a MER caused by sounds that the rat produces (vocalization and chewing) itself. 


\subsubsection{Noise exposed SMs versus control SMs show differences that are less apparent than auditory deprived SMs versus control SMs}

In the noise exposed group mATPase type I fibres were only half in number as seen in the control group. Even when we included the fibres in the type mATPase 'Misc' 1 category, which were also fibres that showed strong IR tot the $\mathrm{mAb}$ to $\mathrm{MHC}$, the percentage (14.3\%) is considerably less than found in the control group $(17.7 \%)(p$-value $=0.001)$. However, the larger number of type mATPase IIA correlates with the anticipated fast to slow shift of the fibres in the noise exposed SMs, at the expense of the number type mATPase IIX fibres. Also the significantly larger number of 'pure' MHC IIA fibres in the group of mATPase type IIA fibres supports our hypothesis. Moreover the overall expression of MHC IIA differed; MHC IIA was found in 52.2\% of the fibres in the noise exposed group and in $40.2 \%$ in the control group ( $p$-values $<0.0005$ ). The muscle fibres in the mATPase IIX category in the noise exposed group significantly showed more co-expression of MHC IIA than in the control group ( $p$-value $<0.0005$ ). The overall expression of MHC IIX did not vary between both groups. However, less 'pure MHC IIX' fibres were counted in the noise exposed group ( $p$-value $<0.0005$ ). Perhaps the shift of fast to slow mainly concerns the type II fibres, involving a shift from IIX to IIA, while in the type I fibres a different type of shift occurs. For instance, the expression of cardiac- $\alpha \mathrm{MHC}$ was noticed more significantly in the noise exposed group than in the control or auditory deprived groups (6\% versus $1.8 \%$ and $2.9 \%$, respectively) (p-value $<0.0005$ ). The cardiac- $\alpha$ MHC is supposed to be much faster than MHC I (VanBuren et al., 1995) and its upregulation is not a typical feature of transforming muscle fibres in the rat (Putman et al., 1999). Cardiac- $\alpha$ MHC upregulation in the noise exposed group may therefore have a functional purpose in typical slow oxidative fibres like the mATPase type I and type 'Misc'1 fibres in supplying them with a higher speed of contraction in combination with more favourable oxidative capabilities.

Thus, a fast to slow shift caused by exposing the rats to noise did not seem to take place as evident as we had been expected. There may be several reasons for this unexpected finding. It can be suggested that the noise stimulus was too weak to test the NIHL protecting function hypothesis effectively. The mATPase fibre composition would have been possibly altered more explicitly towards a slow type fibre shift in the noise exposed group to higher SPL, thereby accepting a risk of inducing some NIHL.

Although the results found in the noise exposed group do not contradict the protection theory, it supports the view that the SM has perhaps another more important function. The results suggest that even in the absence of loud noise the SMs were quite active in the control group, almost as much as the SMs in the noise-exposed group. Contraction of the SM was shown to occur at SPLs much lower than the acoustic reflex threshold in man (Neumann et al., 1996) as well as in rats (Pilz et al., 1997). The small shift in fast to slow muscle fibre types in the SM from the noise exposed group combined with a very clear slow to fast shift in the auditory deprived group, are in accordance with the view that the purpose of the SM is the unmasking of high frequency signals by attenuating low frequency low level noise (Pang and Guinan, 1997) in everyday life.

The SM activity is especially relevant when considering the upward spread of masking phenomenon in the cochlea by low frequency sound. Moreover it was mentioned that the outer hair cells in the cochlea 
function best when not exposed to sounds of SPL above $60 \mathrm{~dB}$, thus a SPL input attenuation filter makes sense (Møller, 2000). The SM activity attenuating the SPL, even below the acoustic reflex threshold, serves to improve the frequency sensitivity and selectivity mediated by the outer hair cell action.

\subsection{Conclusions}

The SM shows an adaptation of fibre composition as a result of staying in an environment with either a noise enhanced level or auditory deprivation obtained by external meatal closure. This finding is comparable to the adaptation as described for skeletal muscles subjected to (endurance) training or muscle unloading experiments.

The SM fibre composition differences between the control and auditory deprived groups confirm the hypothesis that the SM is active in modifying external sound reaching the cochlea. The finding that noise exposition, compared to the control group, alters the SM fibre composition to a limited extent does not discard the hypothesized NIHL protection theory, but it is in favour of the active unmasking function of the SM even in the absence of loud noise even more.

Acknowledgements: We kindly thank A.F.M. Moorman of the Dept. Anatomy/Embryology, University of Amsterdam, and E. Dupont-Versteegden, Dept. Physiology and Biophysics, Little Rock, USA, for the possibility of using the mAbs deployed. This study was supported by the Heinsius-Houbolt Foundation and the Maastricht ENT Research Foundation. 


\section{References}

- $\quad$ Andersen, P., Henriksson, J., 1977. Training induced changes in the subgroup of human type II skeletal muscle fibres. Acta Physiol. Scand. 99 (1), 123-125.

- Berge, H. van den, Wirtz, P., 1989. Detailed morphology of the stapedius muscle of the rat. An integrated light microscopical, morphometrical, histochemical, immunohistochemical and electron microscopical study in relation to function. J. Anat. 166, 157-169.

- $\quad$ Berge, H. van den, Kingma, H., Kluge, C., Marres, E.H.M.A., 1990. Electrophysiological aspects of the middle ear muscle reflex in the rat: Latency, rise time and effect on sound transmission. Hear. Res. 48 (3), 209-219.

- Borg, E., 1980. Recovery of the human intra-aural muscle reflex after "pauses" of various depth. Acta Otolaryngol. 90(1-2), 1-5.

- Borg, E., Nilsson, R., Engström, B., 1983. Effect of the acoustic reflex on inner ear damage induced by industrial noise. Acta Otolaryngol. 96 (5-6), 361-369.

- Borg, E., Counter, S.A., Rosler, G., 1984. Theories about middle ear muscle function. In: Silman, S. (Ed.), The acoustic reflex; Basic principles and clinical applications. Acad. Press, Orlando FI, pp. 50-87.

- Bredman, J.J., Weijs, W.A., Moorman, A.F.M., 1992. Presence of cardiac $\alpha$-myosin correlates with histochemical myosin Ca ${ }^{2+}$ ATPase activity in the rabbit masseter muscle. Histochem. J. 24 (5), 260-265.

- $\quad$ Brooke, M.H., Kaiser, K.K., 1970. Muscle fibre types how many and what kind? Arch. Neurol. 23 (4), 369-379.

- Caiozzo, V.J., 2002. Plasticity of skeletal muscle phenotype: mechanical consequences. Muscle Nerve 26 (6), 740-768.

- Caiozzo, V.J., Baker, M.J., Huang, K., Chou, H., Wu, Y.Z., Baldwin, K.M., 2003. Single-fiber myosin heavy chain polymorphism: how many patterns and what proportion? Am. J. Physiol. Regul. Integr. Comp. Physiol. 285 (3), R570-R580.

- $\quad$ Chalmers, G.R., Roy, R.R., Edgerton, V.R., 1992. Variation and limitations in fiber enzymatic and size responses in hypertrophied muscle. J. Appl. Physiol. 73 (2), 631-641.

- Chillibeck, P.D., Bell, G.J., Socha, T., Martin, T., 1998. The effect of aerobic exercise training on the distribution of succinate dehydrogenase activity throughout muscle fibres. Can. J. Appl. Physiol. 23 (1), 74-86.

- Dammeijer, P.F.M, Mameren van, H., Dijk van, P., Moorman, A.F.M, Habets, P., Manni, J.J., Drukker, J., 2000. Stapedius muscle fibre composition in the rat. Hear. Res. 141 (1-2), 169-179.

- Dammeijer, P.F.M, Schlundt Bodien, Q.C., Chenault, M.N., Manni, J.J., Anteunis, L.J., 2002. Effects of early auditory deprivation and stimulation on auditory brainstem responses in the rat. Acta Otolaryngol. 122 (7), 703-708.

- Dammeijer, P.F.M, Dijk van, P., Manni, J.J., Mameren van, H., 2006. Stapedius muscle fiber characterization during postnatal development in the rat. Hear. Res. 219 (1-2), 48-55.

- Delp, M.D., Pette, D., 1994. Morphological changes during fiber type transitions in low-frequency-stimulated rat fast twitch muscle. Cell Tissue Res. 277 (2), 363-371.

- Demirel, H.A., Powers, S.K., Naito, H., Hughes, M., Coombes, J.S., 1999. Exercise-induced alterations in skeletal muscle myosin heavy chain phenotype: dose-response relationship. J. Appl. Physiol. 86 (3), 1002-1008.

- $\quad$ Ferraro, J.A., Melnick, W., Gerhardt, K.R., 1981. Effects of prolonged noise exposure in chinchillas with severed middle ear muscles. Am. J. Otolaryngol. 2 (1), 13-18.

- Gorza, L., 1990. Identification of a novel type 2 fibre population in mammalian skeletal muscle by combined use of histochemical myosin ATPase and anti-myosin monoclonal antibodies. J. Histochem. Cytochem. 38 (2), 257-265. 
- Guth, L., Samaha, F.J., 1970. Procedure for the histochemical demonstration of actomyosin ATPase. Exp. Neurol. 28 (2), $365-$ 367.

- Henselman, L.W., Henderson, D., Subramaniam, M., Sallustio, V., 1994. The effect of 'conditioning' exposures on hearing loss from impulse noise. Hear. Res. 78 (1), 1-10

- Lucas, C.A., Kang, L.H., Hoh, J.F., 2000. Monospecific antibodies against the three mammalian fast limb myosin heavy chains. Biochem. Biophys. Res. Commun. 272 (1), 303-308.

- Mabuchi, K., Szvetko, D., Pinter, K., Sreter, F.A., 1982. IIB to IIA fiber transformation in intermittently stimulated rabbit muscles. Am. J. Physiol. 242 (5), C373-C381.

- Maier, A., Gambke, B., Pette, D., 1986. Degeneration-regeneration as a mechanism contributing to the fast to slow conversion of chronically stimulated fast-twitch rabbit muscle. Cell Tissue Res. 244 (3), 635-643.

- Møller, A.R., 1965. An experimental study of the acoustic impedance of the middle ear and its transmission properties. Acta Otolaryngol. 60,129-149.

- Møller, A.R., 2000. Hearing: Its Physiology and Pathophysiology, Academic Press, San Diego, CA, pp. 345-368.

- Moorman, A.F.M., Boer, P.A.J. de, Linders, M.Th., Charles, R., 1984. The histone H5 variant in Xenopus laevis. Cell. Differ. 14 (2), $113-123$.

- Neumann, J., Uppenkamp, S., Kollmeier, B., 1996. Detection of the acoustic reflex below 80 dB HL. Audiol. Neurootol. 1(6), 359-369.

- Ogilvie, R.W., Feeback, D.L., 1990. A metachromatic dye-ATPase method for the simultaneous identification of skeletal muscle fibre types I, IIA, IIB and IIC. Stain technol. 65 (5), 231-241.

- $\quad$ Pang, X.D., Guinan, J.J.Jr., 1997. Effects of stapedius-muscle contractions on the masking of auditory-nerve responses. J. Acoust. Soc. Am., 102 (6), 3576-3586.

- Perie, S., Agbulut, O., St Guily, J.L., Butler-Browne, G.S., 2000. Myosin heavy chain expression in human laryngeal muscle fibers. A biochemical study. Ann. Otol. Rhinol. Laryngol. 109(2), 216-220.

- Pette, D., 2002. The adaptive potential of skeletal muscle fibers. Can. J. Appl. Physiol. 27(4), 423-448.

- Pette, D., Staron R.S., 1990. Cellular and molecular diversities of mammalian skeletal muscle fibers. Rev. Physiol. Biochem. Pharmacol. 116: 1-76.

- Pette, D., Staron, R.S., 1997. Mammalian skeletal muscle fiber type transitions. Int. Rev. Cytol. 170, 143-223.

- Pilz, P.K.D., Ostwald, J., Kreiter, A., Schnitzler, H.U., 1997. Effect of the middle ear reflex on sound transmission to the inner ear of the rat. Hear. Res. 105 (1-2), 171-182.

- Putman, C.T., Conjard, A., Peuker, H., Pette, D., 1999. Alpha-cardiac-like myosin heavy chain $\mathrm{MHCl}$ alpha is not upregulated in transforming rat muscle. J. Muscle Res. Cell Motil. 20 (2), 155-162.

- Quaranta, A., Portalatini, P., Henderson, D., 1998. Temporary and permanent threshold shift: an overview. Scand. Audiol. Suppl. $48,75-86$.

- Quiroz-Rothe, E., Rivero, J.L., 2004. Coordinated expression of myosin heavy chains, metabolic enzymes, and morphological features of porcine skeletal muscle fiber types. Microsc. Res. Tech. 65 (1-2), 43-61.

- $\quad$ Rivero, J.L., Talmadge, R.J., Edgerton, V.R., 1998. Fibre size and metabolic properties of myosin heavy chain-based fibre types in rat skeletal muscle. J. Muscle Res. Cell Motil. 19 (7), 733-742. 
- Roy, R.R., Talmadge, R.J., Fox, K.L.M., Edgerton, V.R., 1997. Modulation of MHC isoforms in functionally overloaded and exercised rat plantaris fibers. J. Appl. Physiol. 83 (1), 280-290.

- Sant'Ana Pereira, J.A.A., Haan, de A., Wessels, A., Moorman, A.F.M., Sargeant, A.J., 1995a. The mATPase histochemical profile of rat type IIX fibres: Correlation with MHC immunolabelling. Histochem. J. 27 (9), 715-722.

- Sant'Ana Pereira, J.A.A., Wessels, A., Nijtmans, L., Moorman, A.F.M., Sargeant, A.J., 1995b. New method for the accurate characterisation of single human skeletal muscle fibres demonstrates a relation between mATPase and MHC expression in pure and hybrid fibre types. J. Muscle Res. Cell Motility 16 (1), 21-34.

- Schiaffino, S., Gorza, L., Sartore, S., Saggin, L., Ausini, S., Vianello, M., Gundersen, K., Lomo, T., 1989. Three myosin heavy chain isoforms in type 2 skeletal muscle fibres. J. Muscle Res. Cell Motility 10 (3), 197-205.

- Schiaffino, S., Reggiani, C., 1996. Molecular diversity of myofibrillar proteins: gene regulation and functional significance. Physiol. Rev. 76 (2), 371-423.

- Schuler, M., Pette, D., 1996. Fiber transformation and replacement in low-frequency stimulated rabbit fast-twitch muscles. Cell Tissue Res. 285 (2), 297-303.

- Staron, R.C., Pette, D., 1993. The continuum of pure and hybrid myosin heavy chain based fibre types in rat skeletal muscles. Histochemistry 100 (2), 149-153.

- Stephenson, G.M., 2001. Hybrid skeletal muscle fibres: a rare or common phenomenon? Clin. Exp. Pharmacol. Physiol. 28(8), 692-702

- Talmadge, R., J., 2000. Myosin heavy chain isoform expression following reduced neuromuscular activity: potential regulatory mechanisms. Muscle Nerve 23 (5), 661-679.

- Tikkanen, H.O., Naveri, H.K., Harkonen, M.H., 1995. Alteration of regulatory enzyme activities in fast-twitch and slow-twitch muscles in low intensity endurance trained rats. Eur. J. Appl. Occup. Physiol. 70 (4), 281-287.

- VanBuren, P., Harris, D.E., Alpert, N.R., Warshaw, D.M., 1995. Cardiac V1 and V3 myosins differ in their hydrolytic and mechanical activities in vivo. Cir. Res. 77 (2), 439-444.

- Weiss, A., McDonough, D., Wertman, B., Acakpo-Satchivi, L., Montgomery, K., Kucherlapati, R., Leinwand, L., Krauter, K., 1999. Organization of human and mouse skeletal myosin heavy chain gene clusters is highly conserved. Proc. Natl. Acad. Sci. USA. 96(6), 2958-2963.

- Wieczorek, D.F., Periasamy, M., Butler-Browne, G.S., Whalen, R.G., Nadal-Ginard, B., 1985. Co-expression of multiple myosin heavy chain genes, in addition to a tissue-specific one, in extraocular musculature. J. Cell Biol. 101(2), 618-629.

- Wormald, P.J., Rogers, C., Gatehouse, S., 1995. Speech discrimination in patients with Bell's palsy and a paralysed stapedius muscle. Clin. Otolaryngol. Allied Sci., 20 (1), 59-62.

- Zakrisson, J.E., 1975. The role of the stapedius reflex in post-stimulatory auditory fatigue. Acta Otolaryngol. 79 (1-2), 1-10. 
- 


\subsection{Introduction}

In this chapter we will discuss the features announced in the introduction. The choice of our muscle fibre classification technique of the rat stapedius muscle (SM) will be further exemplified (6.2). An answer will be given whether this technique can be used as a marker to decide at which age the SM is matured (6.4). We explain why the adult SM is showing a rather complex fibre type assembly (6.3).

Before investigating the changes of SM fibre composition that is caused by sound deprivation and noise exposure of the rats growing up, we investigated the developmental associated SM fibre changes to present a fibre composition normally present at different ages. These findings may be of relevance in showing that the SM's maturation is matching the onset of hearing (6.5).

The effects of postnatal auditory deprivation and noise exposure on auditory brainstem responses (ABR) (Chapter 4) will be discussed to establish that the sound characteristics of the physiological noise exposure activated the SM without causing cochlear damage (6.6).

The conclusions that can be drawn considering the function of the SM will be elucidated (6.7).

The value of this experimental study for a better understanding of dysfunction of human hearing will be presented and possible consequences for clinical practice discussed (6.8).

Perspectives for future research will be mentioned (6.9) and our main conclusions are summarized (6.10).

\subsection{Muscle fibre classification technique of the rat SM}

At the start of our research we made choices concerning the technique of muscle fibre analysis. These choices involved the method of identification of the muscle fibre types (6.2.1), and the application of the most meaningful markers to address muscle fibre properties (6.2.2). We discuss the added value of exploring the array of staining combinations of enzyme histochemistry (EHC) and immunohistochemistry (IHC) (6.2.3). We found specific features during the application of the panel of monoclonal antibodies (mAbs); for example, the expression of a myosin heavy chain (MHC) isoform (MHC cardiac- $\alpha$ ) that usually is expressed in cardiac muscle (6.2.4), the uncertainties concerning the immunoreactivity (IR) found to the mAbs specific to MHC IIB (6.2.5), the absence of the neonatal (Neo) MHC isoform in SM fibres (6.2.6) and the abundance of SM fibres that show expression of multiple MHC isoforms (MHC isoform hybridism) (6.2.7).

\subsubsection{The use of serial consecutive cross-sections to characterize the SM}

To a great extent research involving skeletal muscle fibre characterization is performed on the basis of isolated single fibre analysis (gel electrophoresis). Determining the MHC isoform composition of individual muscle fibres is the most accurate method to describe changes in fibre composition, especially in the event of multiple MHC isoform expression within one fibre, also referred to as MHC hybridism. However single fibre analysis is impossible in the SM due to its anatomical features, the muscle being only $1.2 \mathrm{~mm}$ long, with many fibres running obliquely (A.F.M. Moorman, personal communication). 
Furthermore the complexity and diversity of the muscle fibres would require a large number of single fibres to be analysed in order to reveal statistical relevance. Alternatively, analysis of SM homogenates and protein gels would not yield information concerning single fibre MHC isoform hybridism. Thus, with these techniques the myofibrillar adenosine triphosphatase (mATPase) and IHC character and thus the MHC profile of the muscle fibres would not have been described completely.

\subsubsection{The choice of markers}

The performance of the SM depends on the contractile properties of its fibres, such as contraction velocity and fatigability. Although it is generally accepted that while the cross-bridge kinetics are mainly determined by the MHC isoforms present, also other muscle specific proteins can modulate the contractile properties. These can be affected by a mixture of fast and slow myosin light chain (MLC) isoforms (Appendix 2). Although MLC characterization of the SM would have extended the muscle fibre classification, we have not combined MATPase and MHC with MLC characterization. This is because it is impossible in a selected muscle level to obtain the required number of consecutive cross-sections each to be processed for a different monoclonal antibody $(\mathrm{mAb})$ or $\mathrm{mATPase}$ pre-incubation and on top of that still being able to identify all muscle fibres on all cross-sections of that level.

We could have combined for instance IHC with enzymes involved in the energy supply as $\alpha$-glycerophosphate dehydrogenase (GPD) and succinate dehydrogenase (SDH). These enzymes are representatives of anaerobic-glycolytic or aerobic-oxidative ATP production respectively. However they are strongly coordinately regulated with the expression of MHC isoforms (Appendix 2). Because of the mentioned limitation of the number of cross-sections per muscle level we have chosen for combining EHC and $\mathrm{IHC}$ rather than to complete the IHC with examination of cross-sections on the activity of enzymes involved in ATP production. We suppose that the combination of EHC and IHC gives more complementary information (6.2.3) than extension of the fibre characterization with MLC examination or determination of activity of enzymes involved in ATP production.

\subsubsection{Complementary information from mATPase classification and myosin heavy chain monoclonal antibodies}

The mATPase classification which was accomplished by acid, alkali and double pre-incubation mATPase staining is in accordance with the MHC isoform(s) expressed in the muscle fibre. At the time we conducted the study presented in Chapter 2, a mAb specific to MHC IIX was not yet available. Only the mAb to both MHC IIA and IIX (332-3D4) was available. Therefore, in this study it was necessary to use the mATPase double pre-incubation procedure to identify muscle fibres expressing myosin type IIX (Sant'Ana Pereira et al., 1995a). In the studies involving the developmental SM fibre changes and the auditory deprivation and noise exposure (Chapters 3 and 5 ) the mAb specific to MHC IIX had become available to us. However, we still applied the complete mATPase assay involving three different pre-incubations in order to compare earlier data with the results from these later studies. Also in acknowledging that MHC hybrid fibres have mean values of mATPase activity, which relate to shortening velocity intermediate 
in-between their respective pure phenotypes (Sant'Ana Pereira et al., 1995b; Staron and Pette, 1993; Quiroz-Rothe and Rivero, 2004), implementing the same mATPase based classification makes sense in identifying the mATPase 'Misc' types. Thus the mATPase 'Misc' types are hereby representing the fibres with intermediate properties.

We showed that the mATPase category assignment of the SM fibres strongly correlated with the CSA and the MHC isoforms expressed which is strengthening our assumption that intermediate (mostly the 'Misc') SM fibres possess intermediate contractile properties related to the mixture of MHC isoforms (Chapter 5). Fibres in the mATPase type I category were the smallest and mATPase IIX fibres were the largest. Intermediate in size, with some overlapping, were in rank order from small to large, mATPase type 'Misc' 1 (MHC I with MHC IIA), type 'Misc' 3 (MHC IIA with MHC I), mATPase type IIA and type 'Misc' 2 fibres (MHC IIA with MHC IIX). These findings in the SM comply with the observation of Quiroz-Rothe and Rivero (2004) in rat limb muscles.

To continue with utilizing a mATPase classification parallel with a complete IHC panel of specific mAbs to all MHC isoforms provided an indication which MHC is most dominantly expressed in a certain hybrid fibre. This applies for hybrid fibres assigned to the mATPase categories types I, IIA or IIX as well as to the mATPase 'Misc' type categories. In most MHC hybrid muscle fibres assigned to the mATPase types I, IIA and IIX categories it was shown that the most dominant MHC isoforms expressed were the MHC I, IIA or IIX isoform respectively. The mAb IR score was weak to the secondary expressed MHC isoform in most of these fibres; in some however, equally strong IR (+ or ++) of different mAbs was found. This illustrates that the same IR strength of different mAbs (to MHC IIA, IIX or IIB) does not necessarily suggest that equal amounts of MHC isoforms were expressed.

In the study described in Chapter 2, the mAb to both MHC IIA+IIX appeared to be less sensitive than the mAb to only MHC IIA. This was not reported before, possibly because this phenomenon was found by us in only a few EDL fibres and therefore overlooked by others.

Thus complementary IHC with EHC characterization provides a refinement of SM fibre classification which neither of the techniques could have provided on its own.

We support the notion that expression of developmental MHC does not relate to mATPase based classification schemes (Butler-Browne et al., 1984) and therefore MHC isoform classification is necessary to describe developmental muscle fibre type changes. We found in the neonatal rat at PND 7 that the mATPase type IIA muscle fibres were a mixture of the MHC IIX and MHC Emb isoforms and none actually expressed MHC IIA. This illustrates the restriction of the mATPase classification to discriminate the expression of MHC Emb. Possibly the presence of MHC Emb at PND 7 also was the cause of identifying two mATPase types 'Misc' that were not shown at other ages.

\subsubsection{Expression of MHC cardiac- $\alpha$ isoform in the $S M$}

An unexpected finding was that SM fibres showed co-expression of MHC cardiac- $\alpha$ up to PND 84. MHC cardiac- $\alpha$ is an isoform that usually is not found in limb and trunk muscles but is expressed in the atrium of the heart. The presence of MHC cardiac- $\alpha$ in the SM may point to incomplete 
differentiation, as was described for the developing semitendinous muscle of the piglet (Lefaucheur et al., 1995). Bredman et al. (1991) associated this MHC presence in the adult human and rabbit masseter with their branchial origin. MHC cardiac- $\alpha$ is shown to be expressed in mature muscles of mastication (Butler-Browne et al., 1988; Korfage et al., 2000). The contraction velocity of MHC cardiac- $\alpha$ lies in between the velocities of MHC-I and MHC-IIA fibres (Kwa et al., 1995; Sciote and Kentish, 1996) and is therefore considered an intermediate MHC isoform. We conclude that the expression of MHC cardiac- $\alpha$ in the adult SM, a muscle of branchial origin, is part of the variety of MHC isoforms generally present in the SM. It seems not an indication of incomplete differentiation or being in transition during the adaptation of fibre composition.

\subsubsection{IIB or not IIB?}

In Chapter 2 we reported that all SM fibres showed IR (+ or ++ ) to two or more mAbs, one constantly being the mAb to MHC IIB described by Schiaffino et al. (1989). A gradient of IR to the mAb IIB was noticed; about $10 \%$ of the SM fibres stained ++ in contrast to + staining of the remaining fibres. The ++ staining intensity corresponded to the ++ staining in the EDL fibres parallel processed for reference purposes. We suggested a non-specific binding in the (+) staining fibres. In the EDL this non-specific binding was not found. The impression that non-specific binding could have caused all SM fibres being positive for the mAb to MHC IIB raised some doubt whether the SM contained MHC IIB at all. Therefore, a SDS-PAGE analysis of the 6 and 10 weeks old rat SM was carried out. This analysis indisputably showed the expression of MHC IIB in the SM. The same observation of overall weak immunoreactivity with this mAb to MHC IIB in the rat SM was noticed by van den Berge and Wirtz (1989). They considered this as an indication of cross-reactivity of the mAb. This may have been caused by a different post translational modification of the MHC IIB in the SM, which leads to a different IR to its MAb.

In Chapters 3 and 5 a different mAb to MHC IIB from an optimized hybridoma cell line (provided by $\mathrm{E}$. Dupont-Versteegden, Professor Geriatrics and Physiology and Biophysics, Little Rock, USA) was used. This more specific mAb may explain the finding that in the SM at PND 14 nearly 50\% of the fibres showed IR to the mAb specific for MHC IIB and that this percentage decreased to 3\% of the SM fibres at PND 84. Also in all studies no SM fibres were assigned to the mATPase IIB category, besides several at PND 21. This suggests that the MHC IIB expression is of little significance in the SM. It is however upregulated in SM fibres deprived from noise as being part of the slow to fast MHC isoform shift (Chapter 5).

\subsubsection{Determination of neonatal MHC in the rat SM}

We found no positive IR to neonatal MHC in the SM fibres. Immunohistochemical techniques used were verified by staining for Neo MHC on the different muscles at the laboratory of the University of Amsterdam (J.A.M. Korfage). The same results were found. Therefore we concluded that the histochemical technique used was conformed to the standard procedure and that Neo MHC could not be shown in the rat SM by the mAb we used. Neo MHC was shown in other muscles of very young rats up to PND 40 (Di Maso et al. 2000); these authors concluded that the regulation of the Neo MHC and fast type IIB MHC 
isoform are coupled to one another as a down regulation of $\mathrm{Neo} \mathrm{MHC}$ coincided with an up regulation of MHC IIB. We found that the SM fibres already showed an IR to the mAb specific to MHC IIB at PND 7, which is very early. Whether Neo MHC IR could have been present at an earlier developmental period remains speculative.

Jung et al. (2004) demonstrated Neo MHC mRNA transcripts comprising $0.3 \pm 0.07$ percent of total transcripts in the adult rat SM by using competitive polymerase chain reaction (PCR). This does however not mean that Neo MHC mRNA is actually translated at the protein level (Habets et al., 1999).

\subsubsection{MHC isoform hybridism in the SM}

Our first report (Chapter 2) on the MHC hybridism in a very large number of SM fibres in the adult rat was a relative new and unexpected finding. The general MHC hybridism in SM fibres at PND 42 (Chapter 2) was not confirmed in the studies described in Chapters 3 and 5 . The main reason was the erroneous reported general MHC IIB expression. In paragraph 6.2 .5 we suggested that this finding was due to the mAb IIB that was used. In Chapter 3 we showed that from birth to adulthood, the number of muscle fibres in the SM expressing MHC hybridism decreased. Up to the age of PND 42, at least half of the muscle fibres expressed at least two MHC isoforms. At PND 84 still twenty percent of the fibres co-express two MHC isoforms, sometimes even three. The combinations of $\mathrm{MHC}$ isoforms most prevalent were MHC IA with MHC IX and MHC I with MHC IA.

MHC isoform hybridism in the SM may be indicative for an incomplete differentiation as has been reported in skeletal muscles (Whalen et al., 1981; Butler-Browne et al., 1988; Staron and Pette, 1993). However electron microscopy on the SM of the adult rat failed to show features which would indicate incomplete differentiation (Berge and Wirtz, 1989). Moreover, hybrid fibres also appeared in adult skeletal muscles (Larsson and Moss, 1993) and in our co-processed extensor digitorum muscles (EDLs). MHC isoform hybridism is also reported to be present in large quantities especially in other cranial muscles (Sciote et al., 2003).

We conclude that MHC isoform hybridism is a feature of the mature rat SM and therefore is of functional importance. This issue will be addressed in following paragraphs.

\subsection{Does the complex muscle fibre composition in the SM make sense?}

Mammalian skeletal muscles consist of populations of slow-contracting, fatigue-resistant, oxidative fibres that are adapted for slow repetitive or postural type of contractile activity. Also they consist of fast-contracting, glycolytic fibres with a low fatigue resistance, which are recruited for fast phasic movements (Mckoy et al., 2005; Smerdu and Erzen, 2001). These muscle fibre types express different subsets of MHC isoforms as well as different types and levels of metabolic enzymes suited to the type of function they perform. The contraction velocity of fibres depends on their MHC contents 
mostly. When high speeds of contraction are required, these speeds can be produced only by the fibres that contain the fastest MHCs. Furthermore, fast fibres are capable of producing more isometric force and more power than are slow fibres. Generally a muscle can not be optimized simultaneously for very different tasks. For example a muscle that can contract at very high speed cannot perform properly during a prolonged period of time. An example of a rather simple muscle fibre composition is the rat soleus muscle; showing predominantly slow fibres, being activated chronically as a (weight bearing) postural muscle also this is referred to as having a high 'daily duty time' (Hodgson et al., 2005). If in the rat the SM should perform only one specific task we would expect a much more simple composition than we have found.

The complex SM fibre composition as we described in the adult rat may be an indication that this muscle has to perform several tasks for which each separately, different fibre properties are needed. In general, on activation of a muscle, the sequential recruitment of motor units follows the so-called 'size principle' (Henneman et al., 1965). This implies that the smallest, slow, and fatigue resistant motor units are recruited first. They produce small forces. Then with demands on force increasing, the larger, faster, and lesser fatigue resistant motor units are recruited. This may imply that the function of the SM involves a type of task involving fast and swift strong contractile activity but also a slow and less strong activity of longer duration. Moreover fibre MHC hybridism provides for an even smoother transition in contractile velocities when additional motor units are recruited (according to the 'size principle' of Henneman) involving the muscle fibres containing a mixture of $\mathrm{MHC}$ isoforms and therefore exhibit contractile properties intermediate to that of their pure MHC counterparts.

In conclusion, the fibre composition, as is described, from a functional perspective allows the SM to exhibit a wide range of forces, velocities and levels of fatigue resistance, allowing a dynamic and fine tuning of contractile properties.

The impression that activation of the AR through activation of the SM motoneurons does certainly not follow a straightforward (bilateral) "all or nothing" principle, is further supported by inhomogeneous distribution of input from the two cochleas across the stapedius motoneuron pool. This occurs in such a way as to produce a segregation of function; with motoneurons in one brain stem region responding preferentially (or exclusively) to contralateral sound and motoneurons in other regions responding preferentially (or exclusively) to ipsilateral sound (McCue and Guinan, 1988). This may point to a taskrelated recruitment of motor units that requires different fibre types. Whether the 'size' principle or the 'task-related' recruitment of motor units applies to the SM, both principles seem to benefit from the presence of subpopulations of different muscle fibre types.

As stated earlier MHC hybridism may be a characteristic of fibres with a highly adaptive potential. For example hybrid fibres are more suitable to switch phenotype to meet new functional demands (Baldwin and Haddad, 2001). But the MHC hybridism in adult muscle fibres may also minimize the functional consequence or even the necessity of MHC transition when the demands are changing (Caiozzo et al., 2003), this reason for MHC hybridism in the SM may be of functional importance as we found that MHC transitions do occur after noise exposition much less than we expected. 


\subsection{Muscle fibre characterization as a marker of maturation of the SM}

One should discriminate between the term adult in addressing the rat as a whole or concerning a specific group of muscles. The application of the EHC and IHC combination on the EDL at PND 42 and 84 did not show significant differences (Chapter 2). Punkt et al. (2004) state, that rat limb muscles show an adult like fibre characterization that coincides with the time of weaning at PND 21. However, Baldwin and Haddad (2001) classified the rat limb muscles as adult at PND 40. In Chapter 2 we defined the rat SM fibre composition as adult at PND 42. Compared to reports on limb muscles we believe this statement is justified, especially when considering the fast conversion of developmental MHC isoforms into adult type MHCs already at PND 16 (Chapter 3). In Chapter 3 we described that the SM fibre composition at PND 42 and PND 84 show several differences. If we consider the SM fibre composition as adult at PND 42 , what then does explain the differences with the composition at PND 84? Combining the results of Chapters 2 and 3 we demonstrated that in the rat's SM a shift from fast to slow fibre types occurs in the time period from PND 42 to PND 84. The reason for this fast to slow shift perhaps is a feature of further differentiation, maybe related to the acoustical environment where the rats were accommodated.

The decrease of the number of MHC hybrid fibres from nearly 55\% at PND 42 to $21 \%$ at PND 84 suggests a conversion to a steady state functioning of the SM or an adjustment to the acoustics of the environment. The twenty percent MHC hybrid fibres in the SM at PND 84 supports that the MHC hybridism in the rat $\mathrm{SM}$ is rather a quality of an adult muscle adapted to its function than an indication of developmental stage. Therefore the functional significance of $\mathrm{MHC}$ isoform hybridism is strengthened.

The functional significance of MHC hybrid fibres was stressed in studies in cranial muscles (Sciote et al., 2003), especially jaw muscles (Korfage et al., 2005), by stating that such a variety of complex fibre types was fitting them for their roles in vivo. We hypothesize that the fibre composition in the control rats at PND 84 (Chapter 5) reflects a state customized to the monotonous acoustic environment of the animal quarters. This would probably underestimate the MHC hybridism normally present at that age in rats living in a more natural environment.

\subsection{Maturation of the rat SM runs parallel to and precedes that of the auditory system}

We described that after PND 21 modifications in MHC (co-) expression do occur but the gross mATPase based fibre classification composition remains almost the same (Chapter 3 ). At the time the auditory function has reached an adult like sensitivity in the fourth week of gestation (Geal-Dor et al., 1993) we found that the SM fibre composition was differentiated into a near adult like muscle fibre composition. The SM reached its functional adult state more rapidly than other skeletal muscles. These muscles continue to grow and adapt their fibre type composition until the animal reaches adulthood. 
When discussing the meaning of an early developing SM in the newborn rat, it is relevant to address the vulnerability of the neonatal auditory system to loud noise that is described in animals with an immature (altricial) auditory system at birth such as the rat and hamster (Bock and Seifter, 1978; Lenoir et al., 1979). Although in the human newborn the auditory system development is precocious, there is an increased vulnerability to noise in the premature or immature human newborns, both having an immature auditory system. It has been reported that noise of incubators induce hearing loss (NIHL) in immature or premature neonates (Kent et al., 2002; Robertson et al., 2001; Stennert et al., 1978). The SM primordium appears approximately in the 9th week of intra-uterine life (Candiollo and Levi, 1969). In the 29th week of development the human SM is completely differentiated into structures recognizable as muscle fibres, although it's functional development is supposed not to be complete until birth (Whyte et al., 2001). The relation between the maturation of both the auditory function and the SM fibres has not been investigated thus far.

In the rat, the onset of auditory function was reported to begin at PND 12-14 by recording auditory brainstem responses (ABR). The ABR reaches near adult thresholds at about PND 22 (Geal-Dor et al., 1993). The SM's functional importance for the attenuation of noise is demonstrated by the fact that SM maturation proceeds synchronously with the onset of auditory function and even precedes the maturation of the auditory neuronal circuits. The postnatal developing rat with an immature auditory apparatus at birth (Chapter 4) may be compared to the auditory system in the pre-term or immature human neonate (6.8.2).

\subsection{The effect of sound deprivation or noise exposure on ABR}

We studied the muscle fibre composition of the SM of rats at PND 84, which had been exposed to different levels of noise. The control group of rats were accommodated in quarters with ambient noise. Absolute peak latencies and interpeak latencies (IPL) measurements in control, auditory deprived and noise exposed groups, were conducted at PND 84.

\subsubsection{Effect of early auditory deprivation on $A B R$ in the rat}

In the auditory deprivation group, bilateral surgical external meatal closure was performed on PND 21, a time when ABRs in rats have been reported to reach near adult threshold (Geal-Dor et al., 1993). We found that an average bilateral conductive hearing loss of 29.64 SPL (SD 6.64) was achieved (Chapter 4). The extent of auditory deprivation realized by this closure was comparable to reports by others. (Walger et al., 1993; Keilmann, 1993). Our study demonstrated that auditory deprivation enduring 9 consecutive weeks from PND 21 had no statistically significant effect on IPL I-IV. In Chapter 4 we focussed on several possible explanations why our results appeared to be different from previous reports. It seems that the effect of auditory deprivation depends largely on the age at onset. Most likely the rat 
auditory system at PND 21 had passed a critical period, being defined as a period during which the action of a specific condition is required for normal development of the system (Eggermont, 1986).

\subsubsection{Effect of early noise exposure on $A B R$ in the rat}

The rats in the noise exposed group were exposed to a high level of noise starting on PND 21. Exposing rats to noise for 9 consecutive weeks from PND 21 onwards did not have any effect on the ABR recordings. The maturation of the central auditory pathways after PND 21 is apparently insensitive to this level of stimulus. It also did not cause any cochlear damage in the developing rat with an SM with an adult like muscle function, although the SM showed a different outgrowth compared to the control group (Chapter 5).

\subsubsection{Effectively activating the $A R$}

Primary goal of the noise exposure study was to cause an activation of the SM through eliciting an AR. We intended to prevent NIHL as this would change the AR threshold. In the noise exposed rats we found a general shift towards the MHC IIA character. Within the limits set in advance we conclude that the exposition we chose was effective enough to meet with the intentioned SM activation without causing NIHL.

\subsection{SM fibre characterization; its relevancy with respect to SM function}

Three major functions are attributed to the SM. These are (1) injury-prevention, (2) interference and (3) desensitization, and are incorporated in the theory presented by Borg et al. (1984). Furthermore we mentioned the ossicular-chain fixation theory. They were discussed in the general introduction (Chapter 1). Does the described fibre composition make sense when it is related to the proposed functions of the SM? And do our studies on muscle fibre characterization point out the function likely to be the most important?

\subsubsection{Injury-preventing function}

The SM can be described as a fast contracting muscle and is suitable for the cochlea protecting function. The speed of contraction of the SM is supposed to be high because it is part of the acoustic reflex, one of the fastest reflexes in mammals as is found by Pilz et al. (1997). We found that approximately $80 \%$ of SM fibres were fast type II. Even almost half the fibres which contained MHC I were assigned to the mATPase type 'Misc' 1 and co-express MHC IIA. Moreover, a considerable number of these fibres coexpressed MHC cardiac- $\alpha$, that will cause the fibre to contract faster than a pure MHC I fibre.

The SM should not only be fast contracting, it should have a favourable oxidative metabolism in order to prevent AR decay as a result of muscle fatigue. The adult rat SM is composed of nearly twenty percent 
fibres showing strong IR to the mAb specific to MHCI. Myosin types IIA and IIX that combine the features being fast with a high level of fatigue resistance were strongly expressed in the vast majority of the adult SM fibres. We may therefore conclude that the SM is sufficiently equipped to fulfil the proposed protection to $\mathrm{NIHL}$.

\subsubsection{Interference and Desensitization theory}

It is known that in sound exposures above $80 \mathrm{~dB}$ SPL the sensitivity and frequency analysis of the cochlea may be less effective. The SM acts as an input attenuation filter, in maintaining the exposure of sound at the cochlea preferably at levels below $80 \mathrm{~dB} \mathrm{SPL}$, in order to optimize the sensitivity and frequency selectivity of the cochlea that is mediated by the outer hair cell activity.

It is known that in an environment with complex noise the activation of the SM occurs at much lower sound levels than the usually measured ART for pure tones. Modulation of sound conduction at these SPLS of complex noise is supposed to serve an unmasking of high frequency signals in usually low frequent background noise. The most important function of the SM is considered to be the unmasking effect of the AR by attenuating lower frequencies more than the high frequencies (Avan et al. 1992; Wormald et al., 1995; Pang and Guinan, 1997). It was also suggested that high frequency sounds themselves are better transmitted through the middle ear on initial activation of the SM during the AR. Supposedly; this is due to a better "lining up" of the ossicular chain by the SM contraction (Freeman and Sohmer, 1990). Therefore, we assume that at low amplitudes of SM contraction, which occur at SPLs below the ART, there is this better transmission of higher frequencies. The consequence of this SM activity in complex noise at sound intensities below the ART is that the SM is activated much more frequently than if it was only activated by noise above the usually mentioned ART for pure tones. That the SM is activated frequently when exposed to ambient level noise is supported by the general fast to slow shift that takes place in the SM fibres from PND 42 to PND 84. The similarity of SM muscle fibre composition in the SMs exposed to noise compared to the controls (Chapter 5) also supports our view that the SM is frequently activated in the ambient noise levels of the control group. In conclusion, a frequently or continuously active SM also at environments below $80 \mathrm{~dB}$ SPL supports the hypothesis that the main purpose of the $\mathrm{SM}$ is not prevention of NIHL. The SM performs an unmasking function by filtering low frequencies of noise in favour of higher pitched sounds in every day life also in the absence of loud noise.

\subsubsection{Function of the SM in the neonate rat versus the adult; is there a difference?}

In our results on the developmental changes of the rat SM fibre composition, the high speed of reaching maturity is of particular interest. This result illustrates the importance of having a functional SM after birth as soon as possible. Because of the relative higher vulnerability to NIHL in the maturing auditory system a protective function makes sense.

In fact the muscle fibre composition of the SM after PND 16 seems to be ready to perform as a fast and fatigue resistant muscle. At the time of weaning, and later on, leaving the protective environment of the 
mother, it also seems useful to be equipped with an unmasking apparatus that provides an improvement of hearing in terms of coping with the signal/noise ratio, and hereby enhancing its chance of survival. Probably in these early developmental stages the activation of the SM by sounds below the ART for pure tones is an advantage for survival. Therefore also in the very young rat the modulation of external sounds even at ambient noise levels, is served by the already functionally matured SM.

\subsection{Implications for stapedius muscle related clinical issues}

\subsubsection{Increased susceptibility to NIHL having a non-functional SM}

It was mentioned in Chapter 1 that several authors have reported on the increased susceptibility to NIHL coinciding with the loss of a proper SM function, in human as well as in animal studies. In human studies, patients with a paralysis of the facial nerve and patients that underwent a stapedectomy with sectioning the stapedius tendon were analyzed. In the animal studies, the tendon was simply cut before exposure to loud noise started. In our studies, we conclude that the SM fibre composition shows that the rat is equipped with an SM that may perform the protective function. We therefore suggest to caution patients with facial nerve paralysis for an increased susceptibility for acquiring NIHL since these patients also lack the protective function of the SM.

Based on the literature we suggested that the susceptibility to NIHL in man is different between individuals and may be related to the effectiveness of the AR. This also depends on the individual contractile properties of the SM (Chapter 1). In evaluating the SMs in the different groups of rats, we found a very small variability of muscle fibre composition within the same group (Chapter 5 ). This seems contradictory to our suggestion above. However, it is possible that investigating an inbred strain of rats is minimizing the naturally occurring variability of muscle fibre composition in individuals. Also the acoustic load in the noise exposed group may not have been challenging enough for the individual rat to show this variability of $A R$ effectiveness and adaptation of muscle fibre composition. In humans the described differences in individual susceptibility to noise and the development of NIHL might be explained in part by the variability of SM fibre composition and the resulting function.

\subsubsection{Increased susceptibility to NIHL in the premature infant}

Animal studies have demonstrated that noise may cause outer hair cell loss at a significantly lower intensity in neonatal cats and guinea pigs than in their adult counterparts (Falk et al., 1974; Henley and Rybak, 1995) There is also a vulnerability of the rat neonatal auditory system to loud noise, especially due to an immature auditory system during the first four postnatal weeks (Bock and Seifter, 1978; Lenoir et al., 1979). The rapid maturation of the rat SM into a muscle with an adult MHC composition, synchronously, or even preceding the auditory maturation is likely to be of functional significance. This may also be of significance in human neonates. 
It has long been recognized that the human premature or immature newborns have an increased risk to develop NIHL when exposed to a noisy environment such as an incubator (Kent et al., 2002; Robertson et al., 2001; Stennert et al., 1978). Incubator noise, applied to guinea pigs continuously during their second week after birth, was shown to destroy a proportion of the sensory cells in the cochlea histologically. Adult guinea pigs, however, were not vulnerable in this way (Douek et al., 1976). Recent reports suggest noise levels are often still exceedingly high in the neonatal intensive care unit (NICU) and in incubators, despite a noise control protocol (Chang et al., 2006; Kent et al., 2002). Peak noise levels in excess of $120 \mathrm{~dB}$ have been found. Stennert and colleagues (1978) found that the majority of children in their study treated in incubators had audiograms suggestive of NIHL. Premature born neonates, still having an immature cochlea and auditory pathway at term, are susceptible to NIHL even more (Glass, 1999). The maturation of the human SM is normally not complete until birth (Whyte et al., 2001).This may be of significance in the premature or immature neonate, contributing to the increased susceptibility to $\mathrm{NIHL}$.

\subsubsection{Reconstruction of the SM tendon to the stapes prosthesis}

We found that the SM fibre type composition can be influenced by the level and duration of the noise exposure (Chapter 5). This makes it more feasible that the SM is important in noise protection and that the SM is of significance in the interference and desensitization theory (6.7.1 and 6.7.2). The desensitization and interference theory was significantly supported by different reports concerning Bell's palsy patients and stapes replacement surgery in patients with otosclerosis. Chadwell and Greenberg (1979) reported reduced intelligibility of speech in noise in stapedectomized individuals. A decreased loudness discomfort level (LDL) was reported after stapedectomy in otosclerosis patients (Martin and Beck, 1975).

In comparative studies, authors have reported an improved signal to noise ratio, improving discrimination of speech in noise, better discrimination of loud speech and a higher LDL by reconstructing the SM tendon to the stapes piston during otosclerosis surgery. (Arnold et al., 2007; Causse et al., 1997; Colletti et al., 1993; Colletti and Fiorino, 1994; Gros et al., 2000; Rasmy, 1986).

Also, Møller (2000) suggested that patients with stapes prosthesis, and a disabled SM function, may have a higher susceptibility to NIHL. Bull (1966) reported of a patient who developed a pronounced temporary threshold shift (TS) after he returned to work in an environment with loud noise after having experienced a stapedectomy operation including incision of the SM. The hearing loss was somewhat similar in degree for all frequencies and did not show any emphasis around the $4 \mathrm{kHz}$ point. Stapedectomized ears with their SM tendon incised have an altered acoustic impedance of the middle ear and, if acquired, their NIHL is extending below the $4 \mathrm{kHz}$ frequency (Steffen et al., 1963). But do these observations change the way the SM is managed during surgery for otosclerosis?

After successful surgery for otosclerosis patients are probably enjoying their improvement in hearing postoperatively, that their suboptimal discrimination of speech in noise may go unnoticed. What seems to be of value however is to warn these patients about their increased susceptibility to acquire NIHL. 
Since they preoperatively had the relative protection of a conductive hearing loss, following surgery their susceptibility to acquire NIHL is particularly greater than before. It is also greater than in normal hearing people with a functional AR.

Hüttenbrink (2007) stated that surgery for otosclerosis, when the stapes is replaced and the SM is incised, reduces the stiffness of the tympano-ossicular system. Temporal bone experiments showed that elevated static pressures, like in tympanometry, can displace a piston up to $0.5 \mathrm{~mm}$ in the vestibule. These movements, which now are made possible by the missing attachment of the piston to the annular ligament, may explain why a short piston can be lifted out of the footplate perforation (e.g. after sneezing) or a piston with excessive length might come into contact with the membranous labyrinth (Hüttenbrink, 2007). This phenomenon is less likely to occur by preserving the stapedius tendon, contributing a significant degree of stiffness on the conductive system (Colletti et al., 1993).

The question arises whether it makes sense to encourage ear, nose and throat (ENT) surgeons to preserve the SM function in otosclerosis surgery; this technique is after all not adopted in the Netherlands. If the procedure of reconstructing the tendon of the SM to the stapes prosthesis is not hazardous or if it does not jeopardize the outcome of surgery and if it can readily be performed by an experienced ENT surgeon, it should be better to reconstruct the SM tendon to the prosthesis, preserving a regular SM function. This opinion was shared by Silverstein et al. (1999). In conclusion, we support preserving the SM function during otosclerosis surgery.

\subsection{Future research perspectives}

There still remain many questions to answer. Does the auditory deprivation or noise exposure have the same effect on the rat's SM fibre composition when the experiment is performed at adult age? And, will there be a significant change of SM fibre types when stimulating at a sound levels higher than $80 \mathrm{~dB}$ SPL? It would be of interest to investigate the ABRs at PND 84 in different groups of rats exposed to the same level of noise from PND 21 onwards; a group having the SM tendons severed at PND 21 and a control group that underwent a sham operation.

Investigating the TTM muscle fibre composition in the normal, auditory deprived and noise exposed rats may illustrate the absence or presence of a significant purpose in relation to auditory exposition.

It would be of value to investigate prospectively the effects of SM function rehabilitation during surgery for otosclerosis. 


\subsection{General Conclusions}

1. The application of the EHC and IHC combination on the EDL shows the functional character of a hybrid fibre in terms of myosin dominance, which is necessary to understand the functional character of the SM. Complementary IHC with EHC characterization provides a refinement of SM fibre classification which neither of the techniques could have provided on its own.

2. In the SM of the rat, the adult like MHC isoform composition develops rapidly, even faster than in diaphragm and skeletal muscles. The adult muscle phenotype composition is completed in the third postnatal week. This development runs synchronously with the onset of auditory function at the same postnatal moment. This supports our view of the SM's functional importance to control the presented noise.

3. The SM fibre composition is different from that what has been previously described. The mATPase type IIA fibres were not the prevalent type as was suggested by previous reports. Myosin ATPase type IIX fibres dominate. Compared with limb and trunk muscles, the rat's SM shows a highly unusual composition. In addition to the normal slow, type I, and fast, type II fibres, it contains a MHC isoform composition which is typical for cardiac muscle. Another remarkable feature is that many of the fibres are hybrid and sometimes even co-express three $\mathrm{MHC}$ isoforms (for example, MHC I+\|A + cardiac- $\alpha$ or MHC $\|\mathrm{A}+\| \mathrm{X}+\| \mathrm{B}$ ).

4. The predominance of myosin type II fibres provides the SM the capability to react fast (high Vmax). Myosin types II that are associated best with fatigue resistance were found in the majority of the fibres. Therefore, the SM in the adult rat is well equipped to protect auditory receptors from injury and may constantly work within the scope of the interference and desensitization theory.

5. The MHC hybridism in the rat SM represents rather the quality of a full-grown muscle adapted to its function than a reflection of a development stage. The range of physiological characteristics, dependent on the composition of myosin isoforms, in adult skeletal hybrid muscle fibres may have functional significance.

6. From PND 21 up to PND 84 MHC hybridism in the SM decreases, which may be an expression of structurally consolidating the adult functional state reached at PND 21. Further adaptation is influenced largely by the acoustical environment.

7. The rat SM displays a large variety of different muscle fibre types varying from pure MHC I type fibres to mATPase type IIX fibres co-expressing MHC IIB. Whether the size principle or the principle of task-related recruitment of motor units applies on SM activation, both principles seem to benefit from the presence of these subpopulations of muscle fibres. It supports the hypothesis that the SM has different tasks to perform.

8. The SM shows an adaptation of fibre composition depending on different external sound environments. The tested environments were noise enhanced levels or moderate auditory deprivation obtained by external meatal closure. This SM adaptation is comparable to the 
adaptation as has been described earlier for skeletal muscles subjected to (endurance) training or muscle unloading experiments.

9. The SM fibre composition differences between the control and auditory deprived groups confirm our hypothesis that the SM is active in modifying external sound transmitted to the cochlea. The finding that noise exposition, compared to the control group, alters the SM fibre composition to a limited extent does not discard the hypothesized NIHL protection theory, but it is in favour of the active unmasking function of the SM even in the absence of loud noise even more.

10. If the procedure of reconstructing the tendon of the SM to the stapes prosthesis is not hazardous or it does not jeopardize the outcome of surgery and can readily be performed by an experienced ENT surgeon, this probably should be pursued in otosclerosis surgery.

11. The increased susceptibility to NIHL in premature newborn humans may at least to a certain extent be due to an ineffective AR. 


\section{References}

- Arnold, A., Blaser, B., Hausler, R., 2007. Audiological Long-Term Results following Stapedotomy with Stapedial Tendon Preservation. Adv. Otorhinolaryngol. 65, 210-214.

- Avan, P., Loth, D., Menguy, C., Teyssou, M., 1992. Hypothetical roles of middle ear muscles in the guinea-pig. Hear. Res. 59(1), 59-69.

- Baldwin, K.M., Haddad, F., 2001. Plasticity in Skeletal, Cardiac, and Smooth Muscle, Invited Review: Effects of different activity and inactivity paradigms on myosin heavy chain gene expression in striated muscle. J. Appl. Physiol. 90, 345-357.

- Berge, H. van den, Wirtz, P., 1989. Detailed morphology of the stapedius muscle of the rat. J. Anat. 166, 157-169.

- $\quad$ Bock G.R., Seifter, E.J., 1978. Developmental changes of susceptibility to auditory fatigue in young hamsters. Audiology 17(3), 193-203.

- Borg, E., Counter, S.A., Rosler, G., 1984. Theories about middle ear muscle function. In: Silman, S. (Ed.), The acoustic reflex; Basic principles and clinical applications. Acad. Press, Orlando Florida, pp. 50-87.

- Bredman, J.J., Wessels, A., Weijs, W.A., Korfage, J.A., Soffers, C.A., Moorman, A.F., 1991. Demonstration of cardiac-specific myosin heavy chain in masticatory muscles of human and rabbit. Histochem. J. 23, 160-170.

- Bull, T. R., 1966. Noise trauma deafness after stapedectomy with recovery. J. Laryngol. 80, 631-633

- $\quad$ Butler-Browne, G.S., Whalen, R.G., 1984. Myosin isozyme transitions occurring during the postnatal development of the rat soleus muscle. Dev. Biol. 102(2), 324-334.

- Butler-Browne, G.S., Eriksson, P.O., Laurent, C., Thornell, L.E., 1988. Adult human masseter muscle fibres express myosin isozymes characteristics of development. Muscle Nerve 11, 610-620.

- Caiozzo, V.J., Baker, M.J., Huang, K., Chou, H., Wu, Y.Z., Baldwin, K.M., 2003. Single-fiber myosin heavy chain polymorphism: how many patterns and what proportion? Am. J. Physiol. Regul. Integr. Comp. Physiol. 285(3), R570-R580.

- Candiollo, L., Levi, A.C., 1969. Studies on the morphogenesis of the middle ear muscles in man. Arch. Klin. Exp. Ohren Nasen Kehlkopfheilkd. 195 (1), 55-67.

- $\quad$ Causse, J.B., Vincent, R., Michat, M., Gherini, S., 1997. Stapedius tendon reconstruction during stapedotomy: technique and results. Ear Nose Throat J. 76(4), 256-568.

- $\quad$ Chadwell, D.L., Greenberg, H.J., 1979. Speech intelligibility in stapedectomized individuals. Am. J. Otol. 1(2), 103-108.

- Chang, Y.J., Pan, Y.J., Lin, Y.J., Chang, Y.Z., Lin, C.H., 2006. A noise-sensor light alarm reduces noise in the newborn intensive care unit. Am. J. Perinatol. 23(5), 265-271.

- Colletti, V., Fiorino, F.G., 1994. Stapedotomy with stapedius tendon preservation: technique and long-term results. Otolaryngol Head Neck Surg. 111(3 Pt 1), 181-188.

- Colletti, V., Fiorino, F.G., Sittoni, V., Policante, Z., 1993. Mechanics of the middle ear in otosclerosis and stapedoplasty. Acta Otolaryngol. 113(5), 637-641.

- Di Maso, N.A., Caiozzo, V.J., Baldwin, K.M., 2000. Single-fiber myosin heavy chain polymorphism during postnatal development: modulation by hypothyroidism. Am. J. Physiol. Regul. Integr. Comp. Physiol. 278(4), R1099-R1106.

- Douek, E., Dodson, H.C., Bannister, L.H., Ashcroft, P., Humphries, K.N., 1976. Effects of incubator noise on the cochlea of the newborn. Lancet 2(7995), 1110-1113.

- Eggermont, J.J., 1986. Defining and determining sensitive periods. Acta. Otolaryngol. Suppl. 429, 5-9. 
- Falk, S.A., Cook, R.O., Haseman, J.K., Sanders, G.M., 1974. Noise-induced inner ear damage in newborn and adult guinea pigs. Laryngoscope 84(3), 444-453.

- Freeman, S., Sohmer, H., 1990. The influence of sound stimulus parameters on the acoustic reflex waveform. Eur. Arch. Otorhinolaryngol. 247(2), 104-108.

- Geal Dor, M., Freeman, S., Li, G., Sohmer, H., 1993. Development of hearing in neonatal rats: air and bone conducted ABR thresholds. Hear. Res. 69, 236-242.

- Glass, P., 1999. The vulnerable neonate and the neonatal intensive care environment. In: Avery, G.B., Fletcher, M.A. (Eds). Neonatology: pathophysiology and management of the newborn. J.B. Lippincott, Philadelphia, pp. 91-108.

- Gros, A., Zargi, M., Vatovec, J., 2000. Does it make sense to preserve the stapedial muscle during surgical treatment for otosclerosis? J. Laryngol. Otol. 114(12), 930-934.

- Habets, P.E., Franco, D., Ruijter, J.M., Sargeant, A.J., Sant'Ana Pereira, J.A., Moorman, A.F., 1999. RNA content differs in slow and fast muscle fibers: implications for interpretation of changes in muscle gene expression. J. Histochem. Cytochem. 47(8), 9951004.

- Henley, C.M., Rybak, L.P., 1995. Ototoxicity in developing mammals. Brain Res. 20, 68-90.

- Henneman, E., Somjen, G., Carpenter, D.O., 1965. Excitability and inhibitability of motoneurons of different sizes. J. Neurophysiol. 28(3), 599-620.

- Hodgson, J.A., Roy, R.R., Higuchi, N., Monti, R.J., Zhong, H., Grossman, E., Edgerton, V.R., 2005. Does daily activity level determine muscle phenotype? J. Exp. Biol. 208(19), 3761-3770.

- Huttenbrink, K.B., 2007. Clinical significance of stapedioplasty biomechanics: swimming, diving, flying after stapes surgery. Adv. Otorhinolaryngol. 65, 146-149.

- Jung, H.H., Han, S.H., Nam, S.Y., Kim, Y.H., Kim, J.L., 2004. Myosin heavy chain composition of rat middle ear muscles. Acta Otolaryngol. 124(5):569-573.

- Keilmann A., 1993. Einfluss einer Schalldeprivation auf die Hörbahnreifung bei der Ratte. Laryngorhinootologie 72, 15-18.

- Kent, W.D., Tan, A.K., Clarke, M.C., Bardell, T., 2002. Excessive noise levels in the neonatal ICU: potential effects on auditory system development. J. Otolaryngol. 31(6), 355-360.

- Korfage, J.A., Koolstra, J.H., Langenbach, G.E., van Eijden, T.M., 2005. Fiber-type composition of the human jaw muscles - (part 2) role of hybrid fibers and factors responsible for inter-individual variation. J. Dent. Res. 84(9), 784-793.

- Korfage, J.A., Van Eijden, T.M., 2000. Myosin isoform composition of the human medial and lateral pterygoid muscles. J. Dent. Res. 79(8), 1618-1625.

- Kwa, S.H., Weijs, W.A., van Eijden, T.M., 2002. Effects of activation rate on contractile properties of rabbit masseter motor units. Exp. Brain Res. 142(2), 221-226.

- Larsson, L., Moss, R.L., 1993. Maximum velocity of shortening in relation to myosin isoform composition in single fibres from human skeletal muscles. J. Physiol. 472, 595-614.

- Lefaucheur, L., Edom, F., Ecolan, P., Butler-Browne, G.S., 1995. Pattern of muscle fibre type formation in the pig. Dev. Dyn. 203, 27-41.

- Lenoir, M., Bock, G.R., Pujol, R., 1979. Supra-normal susceptibility to acoustic trauma of the rat pup cochlea. J. Physiol. (Paris) 75(5), 521-524. 
- Martin G., Beck C., 1975. Untersuchungen über die Unbehaglichkeitsschwelle des stapedektomierten Ohres. Ein Beitrag zur Funktion des M. stapedius. Arch. Otorhinolaryngol. 209(2), 107-111.

- McCue, M.P., Guinan, J.J., 1988. Anatomical and functional segregation in the stapedius motoneuron pool of the cat. J. Neurophysiol. 60(3), 1160-1180.

- Mckoy, G., Hou, Y., Yang, S.Y., Vega Avelaira, D., Degens, H., Goldspink, G., Coulton, G.R., 2005. Expression of Ankrd2 in fast and slow muscles and its response to stretch are consistent with a role in slow muscle function. J. Appl. Physiol. 98(6), 2337-2343.

- Møller, A.R., 2000. Hearing: Its Physiology and Pathophysiology, Academic Press, San Diego, CA, p. 390.

- $\quad$ Pang XD, Guinan JJ Jr., 1997. Effects of stapedius-muscle contractions on the masking of auditory-nerve responses. J. Acoust. Soc. Am. 102(6), 3576-3586.

- Pilz, P.K.D., Ostwald, J., Kreiter, A., Schnitzler, H., U., 1997. Effect of the middle ear reflex on sound transmission to the inner ear of the rat. Hear. Res. 105, 171-182.

- Punkt, K., Naupert, A., Asmussen, G., 2004. Differentiation of rat skeletal muscle fibres during development and ageing. Acta Histochem. 106(2), 145-154.

- $\quad$ Quiroz-Rothe, E., Rivero, J.L., 2004. Coordinated expression of myosin heavy chains, metabolic enzymes, and morphological features of porcine skeletal muscle fiber types. Microsc. Res. Tech. 65(1-2), 43-61.

- Rasmy, E., 1986. Stapedius reflex after stapedectomy with preservation of the stapedius tendon. J. Laryngol. Otol. 100(5), 521527.

- Robertson, A., Stuart, A., Walker, L., 2001. Transmission loss of sound into incubators: implications for voice perception by infants. J. Perinatol. 21(4), 236-241.

- Sant'Ana Pereira, J.A.A., Haan, de A., Wessels, A., Moorman, A.F.M., Sargeant, A.J., 1995a. The mATPase histochemical profile of rat type IIX fibres: Correlation with MyHC immunolabelling. Histochem. J. 27, 715-722.

- Sant'Ana Pereira, J.A.A., Wessels, A., Nijtmans, L., Moorman, A.F.M., Sargeant, A.J., 1995b. New method for the accurate characterisation of single human skeletal muscle fibres demonstrates a relation between mATPase and MyHC expression in pure and hybrid fibre types. J. Muscle Res. Cell Motility 16, 21-34.

- $\quad$ Schiaffino, S., Gorza, L., Sartore, S., Saggin, L., Ausini, S, Vianello, M., Gundersen, K., Lomo, T., 1989. Three myosin heavy chain isoforms in type 2 skeletal muscle fibres. J. Muscle Res. Cell Motility 10, 197-205.

- Sciote, J.J., Horton, M.J., Rowlerson, A.M., Link, J., 2003. Specialized cranial muscles: how different are they from limb and abdominal muscles? Cells Tissues Organs. 174(1-2), 73-86.

- Sciote, J.J., Kentish, J.C., 1996. Unloaded shortening velocities of rabbit masseter muscle fibres expressing skeletal or alphacardiac myosin heavy chains. J. Physiol. 492 (Pt 3), 659-667.

- Silverstein, H., Hester, T.O., Deems, D., Rosenberg, S., Crosby, N., Kwiatkowski, T., 1999. Outcomes after laser stapedotomy with and without preservation of the stapedius tendon. Ear Nose Throat J. 78(12), 923-928.

- Smerdu, V., Erzen, I., 2001. Dynamic nature of fibre-type specific expression of myosin heavy chain transcripts in 14 different human skeletal muscles. J. Muscle Res. Cell Motil. 22, 647-655.

- Staron, R.C., Pette, D., 1993. The continuum of pure and hybrid myosin heavy chain based fibre types in rat skeletal muscles. Histochemistry 100, 149-153.

- Steffen, T.N., Nixon, J.C., Glorig, A., 1963. Stapedectomy and noise. Laryngoscope 78, 1044-1060. 
Chapter 6

- Stennert, E., Schulte, F.J., Vollrath, M., Brunner, E., Frauenrath, C., 1978. The etiology of neurosensory hearing defects in preterm infants. Arch. Otorhinolaryngol. 221(3), 171-182.

- Walger, M., Laska, M., Schneider, I., Diekmann, H., von Wedel, H., 1993. Maturation of auditory evoked potentials in young guinea pigs with binaural conductive hearing loss. Eur. Arch. Otorhinolaryngol. 250, 362-365.

- Whalen, R.G., Sell, S.M., Butler-Browne, G.S., 1981. Three myosin heavy-chain isozymes appear sequentially in rat muscle development. Nature 292, 805-809.

- Whyte, J., Cisneros, A., Yus, C., Blasco, J., Torres, A., Sarrat, R., 2001. Contribution to the development of the stapedius muscle structure in human fetuses. Anat. Histol. Embryol. 30(3), 175-178.

- Wormald, P.J., Rogers, C., Gatehouse, S., 1995. Speech discrimination in patients with Bell's palsy and a paralysed stapedius muscle. Clin. Otolaryngol. Allied Sci. 20(1), 59-62. 


\section{Summary}

It is still a subject of debate in which manner the stapedius muscle (SM) modulates the acoustical energy in its way through the middle ear towards the cochlea and what its purpose may be. It is the aim of this thesis to contribute to the understanding of the function of the SM. Three major functions usually are attributed to the SM activity; they are incorporated in the injury preventing, interference and desensitization theory. The ossicular-chain fixation theory is also mentioned, which includes that middle ear (MEM) contraction serves to control the position of the ossicles for optimal sound transmission. The tensor tympani muscle is not part of the present thesis. Besides describing the aims and design of our study, the gross anatomy of the middle ear, auditory system and proposed functions of the SM are addressed in Chapter 1.

In Chapter 2 we present the myosin composition of SM fibres of the rat, using consecutive complete SM cross-sections, which were processed by enzymehistochemistry $(\mathrm{EHC})$ to determine acid/alkali lability of myofibrillar adenosine triphosphatase (mATPase) or by immunohistochemistry (IHC) using myosin heavy chain (MHC) antibodies. Fibres were assigned to different mATPase categories on the basis of combinations of staining intensities. Fibres that did not meet the criteria of the mATPase categories type I, IIA, IIX or IIB were assigned to type 'Misc' (miscellaneous) categories. Method accuracy was determined in co-processed extensor digitorum longus (EDL). Per mATPase category the fibres were attributed to groups with specific MHC compositions. It was shown that IHC is complementary to EHC to detect amounts of myosin isoforms that are not detected by EHC. In turn, EHC is able to determine the MHC isoform expressed most dominantly. SM fibres were assigned to the mATPase type IIA category in about 25\%, to the type IIX in 60\% and 15\% to a 'Misc' category (in which most fibres expressed MHC I). All SM fibres expressed two or more MHC isoforms, with a weak immunoreactivity (IR) to MHC IIB, and an intermediate to strong IR to MHC IIA and/or IIX in most fibres. These findings confirm the hypothesis that most SM fibres have the capacity to contract fast and have the better fatigue resistance.

In Chapter 3 we describe developmentally associated changes of myosin composition of SM fibres. We used consecutive complete SM cross-sections (taken from rats on post natal days (PNDs) 7, 14, 16, 21, 28,42 and 84. The muscle fibre classification method applied was essentially the same as used in Chapter 2. Neonatal MHC expression could not be documented with the mAb used. Embryonal (Emb) MHC was expressed at PND 7, very little at PND 14 and none at later PND. Expression of MHC IIB, which was present in almost 50\% of the fibres at PND 7 and 14, diminished to 3\% at PND 84. A decrease in the number of MHC hybrid fibres was found. These results show that the SM is a precociously developing muscle, moreover that the expression of the adult MHC isoform phenotype coincides with the onset of auditory function in the third postnatal week. 
In Chapter 4 we describe the effect of auditory sound deprivation and stimulation on brain stem auditory evoked responses (ABRs) during the maturation period of the rat auditory system. At PND 21, rats were categorized in 3 groups: an auditory deprivation group in which a bilateral average conductive hearing loss of $27 \mathrm{~dB}$ was surgically induced, an auditory activation group exposed to 65-90 dB sound pressure level and a control group. ABR recordings were performed on PND 84. No differences were observed in the inter peak latencies (IPLS) of peaks I-IV between the three groups. Possible confounding factors explaining the discrepancy between these results and those of other animal studies were reviewed. We conclude that the noise exposition did not cause injury to the cochlea nor did the sound deprivation hinder the maturation of the rat auditory system.

In Chapter 5 we report on investigations concerning adaptation of SM fibre type composition comparable to those in skeletal muscle; interventions unloading the skeletal muscle cause slow-to-fast MHC conversions, whereas fast-to-slow conversions occur when the muscle is engaged in resistance training and endurance exercise. We investigated the difference in myosin composition of muscle fibres from SMs of rats exposed to noise, from auditory deprived rats and from rats exposed to low-level ambient noise (control group). A slow to fast shift after sound deprivation (SM unloading) suggests that the SM needs a certain degree of daily activity evoked by environmental sound to sustain its normal composition. Findings are also supportive of the idea that the SM is frequently active in the control group, where ambient noise did not exceed the $80 \mathrm{~dB}$ sound pressure level (SPL). The injury preventing purpose of the SM was supported to some extent by showing changes of SM fibre composition when the rats were exposed to longstanding noise (SM training). Our results are in favour of another postulated function of the $\mathrm{SM}$; the unmasking of high frequency signals in low frequency background noise in every day life.

Finally, Chapter 6 reflects on the method of identification of muscle fibres in the SM and on applying the most meaningful markers to address muscle fibre properties. Furthermore, peculiarities encountered when examining the $\mathrm{SM}$, involving widespread $\mathrm{MHC}$ hybridism and presence or either absence of certain $\mathrm{MHC}$ isoforms, is discussed. We suggest a functional purpose of the complex SM fibre composition and MHC hybridism. The fast maturing of the neonatal rat SM, exemplifies the functional relevance of the SM, which is discussed relating it to the increased susceptibility to noise induced hearing losS (NIHL) in the premature human infant. The findings from the noise exposition and auditory deprivation studies compared to the control group are in favour of an SM having a high daily activity level even in the absence of high noise levels that is supportive of the interference and desensitization function. These sound modulating functions and the increased susceptibility to NIHL possibly caused by loss of SM function, as is documented in literature, are in favour of re-addressing the preservation of SM function in surgery for otosclerosis. 


\section{Samenvatting}

Het is nog steeds een onderwerp van discussie op welke manier de musculus stapedius (SM) het geluid op zijn weg door het middenoor richting slakkenhuis moduleert en wat daar de bedoeling van kan zijn. Het is het doel van dit proefschrift om bij te dragen tot het beter begrijpen van de functie van de SM. Drie belangrijke functies worden gewoonlijk toegeschreven aan de activiteit van de SM; zij maakt deel uit van de theorie van preventie van binnenoorbeschadiging, interferentie en over-stimulatie in het kader van de perceptie van geluid. In de literatuur wordt ook de "gehoorbeentjes fixatie theorie" beschreven die inhoud dat contractie van de middenoorspieren de positie van de gehoorbeentjes controleert ten behoeve van optimale voortgeleiding van geluid. De musculus tensor tympani (TM) maakt geen onderdeel uit van dit proefschrift. Naast de doelstellingen en de opzet van onze studie, zijn de globale anatomie van het middenoor, het auditieve systeem en de voorgestelde functies van de SM besproken in Hoofdstuk 1.

In Hoofdstuk 2 beschrijven we de spiervezelsamenstelling van de SM in de rat. Direct opeenvolgende dwarsdoorsneden van de SM werden bewerkt met mATPase enzymhistochemie (EHC), of met immunohistochemie (IHC). De nauwkeurigheid van de methodiek werd bepaald door synchroon met de coupes van de SM, coupes van een bekende spier, de extensor digitorum longus (EDL), te verwerken. De vezels werden op basis van combinaties van diverse myofibrillaire adenosine triphosphatase (mATPase) bewerkingen ingedeeld in verschillende mATPase categorieën. De vezels die niet voldeden aan de criteria van de mATPase categorie type I, IIA, IIX of IIB, werden toegewezen aan een mATPase categorie type 'Diverse' (in het Engels; miscellaneous, afgekort 'Misc'). In iedere mATPase categorie werden de spiervezels vervolgens ingedeeld in subgroepen met ieder een specifieke samenstelling van het soort zware keten van het myosine eiwit (in het Engels: myosin heavy chain, afgekort MHC). Van het MHC bestaan verschillende moleculaire vormen (isovormen); MHC I, IIA, IIX, IIB en meer. De in de spiervezel aanwezige $\mathrm{MHC}$ isovormen werden geïdentificeerd door de immunoreactiviteit (IR) van specifieke tegen verschillende MHC isovormen opgewekte monoklonale antilichamen (mAbs) te bepalen. De spiervezels van de SM werden in ongeveer 25\% toegewezen aan de mATPase type IIA categorie, in 60\% aan mATPase type IIX en in 15\% aan een mATPase categorie 'Misc.' De SM spiervezels bevatten twee of meer MHC isovormen. De meeste vezels tonen een zwakke immunoreactiviteit (IR) voor de mAb tegen MHC IIB en een gemiddelde tot sterke IR voor de mAb tegen MHC IIA en/of IIX. Deze bevindingen bevestigen de hypothese dat de meeste SM vezels de capaciteit hebben snel te contraheren en dat ze een relatief groot uithoudingsvermogen bezitten.

In Hoofdstuk 3 beschrijven we de veranderingen in myosine samenstelling van spiervezels van de SM tijdens de postnatale ontwikkeling van de rat. Gebruikmakend van de eerder toegepaste methode uit Hoofdstuk 2 werd de spiervezeltype samenstelling beschreven van de SM van ratten op een aantal dagen 
na de geboorte (in het Engels postnatal day, afgekort PND); 7, 14, 16, 21, 28, 42 en 84. De neonatale $\mathrm{MHC}$ isovorm kon met het door ons gebruikte mAb niet worden aangetoond. De Embryonale (Emb) MHC isovorm werd tot aan PND 14 gevonden. De op mATPase-gebaseerde classificatie toonde geen grote wijzigingen na PND 21. Aanwezigheid van de MHC IIB isovorm, die in bijna 50\% van de vezels bij PND 7 en 14 aanwezig was, verminderde tot 3\% bij PND 84, tevens zagen wij een daling van het aantal MHC hybride vezels. Deze resultaten tonen aan dat de SM een relatief vroegrijpe spier is en dat de expressie van het volwassen MHC isovorm fenotype van de SM samenvalt met het begin van de auditieve functie in de derde postnatale week.

In Hoofdstuk 4 beschrijven we het effect van auditieve deprivatie en geluidsstimulatie op de auditief opgewekte hersenstam potentialen (in het Engels auditory brainstem respons, afgekort $A B R$ ) tijdens de rijpingsperiode van het auditieve systeem in de neonatale rat. Op PND 21, werden veertig pasgeboren ratten ingedeeld in 3 groepen; een auditieve deprivatie groep, een lawaai expositie groep en een controle groep. De ABRs werden verkregen op PND 84. Er werden geen verschillen waargenomen in interpiek latentietijden (IPLs) van pieken I-IV tussen de drie groepen. Er is een discrepantie tussen de door ons gevonden resultaten en die van vergelijkbare studies in de literatuur. De hiervoor mogelijk verantwoordelijke factoren werden geëvalueerd. Wij constateren dat de lawaai-expositie geen beschadiging aan het slakkenhuis heeft veroorzaakt (in het Engels noise induced hearing loss, afgekort $\mathrm{NIHL}$ ) en ook dat de geluidsdeprivatie de rijping van het auditieve systeem in de ratten niet heeft belemmerd.

In Hoofdstuk 5 rapporteren we over het onderzoek betreffende veranderingen van de spiervezeltype samenstelling in de SM vergelijkbaar met veranderingen in skeletspieren, zoals die beschreven zijn in de literatuur; het niet gebruiken van de skeletspier leidt tot een verschuiving van langzame naar snellere MHC isovormen in de spiervezels, terwijl een verschuiving van snelle naar langzamere MHC isovormen wordt gezien wanneer de spier intensief wordt gebruikt. Wij onderzochten het verschil in spiervezeltype samenstelling tussen SMs afkomstig van ratten die aan lawaai werden blootgesteld, van auditief gedepriveerde ratten en van ratten die aan het normale omgevingsgeluid in de proefdiervoorziening werden blootgesteld (controle groep). De langzame naar snellere MHC isovorm verschuiving na geluidsdeprivatie (ontlasten van de SM) pleit ervoor dat de SM een bepaalde mate van dagelijkse activiteit, uitgelokt door extern omgevingsgeluid, nodig heeft om zijn normale spiervezeltype samenstelling te behouden. Dit ondersteunt de veronderstelling dat de SM veelvuldig actief is in de controle groep, waar het geluidsniveau de $80 \mathrm{~dB}$ niet overschreed. De hypothese dat de SM bescherming biedt tegen NIHL werd enigszins gesteund doordat de veranderingen die werden aangetoond in de SM spiervezeltype samenstelling na langdurig lawaai expositie, overeenkomen met de veranderingen die in skeletspieren beschreven zijn na trainingsexperimenten. Onze resultaten pleiten ook voor een andere al eerder veronderstelde functie van de SM, namelijk het demaskeren van hoog frequente signalen in laag frequent achtergrondgeluid. 
Tot slot wordt in Hoofdstuk 6 ingegaan op de methodiek van identificatie van de spiervezels in de SM en op het gebruik van de enzym- en immunohistochemistische parameters waarmee een weerspiegeling van de eigenschappen van de spiervezel werd verkregen. De combinatie van IHC en EHC verschafte een verfijning van de SM vezelclassificatie die geen van beide technieken alléén verstrekt kon hebben. Voorts werden onverwachte bevindingen van spiervezeltype samenstelling besproken zoals het wijdverspreide MHC hybridisme en de aanwezigheid van, of juist de afwezigheid van bepaalde MHC isovormen. De complexe spiervezeltype samenstelling van de SM heeft een functionele betekenis en het snelle rijpen van de SM in de pasgeboren rat, die zelfs voorloopt op de rijping van het auditieve systeem, lijkt de functionele relevantie van de SM te benadrukken. In dit verband is ook de verhoogde gevoeligheid voor $\mathrm{NIHL}$ in de humane premature neonaat besproken Het vergelijken van de bevindingen in SMs van ratten die werden blootgesteld aan lawaai en in SMs van de auditief gedepriveerde ratten met de bevindingen in de SMs van ratten in de controle groep duiden erop dat de SM dagelijks een hoog activiteit niveau heeft, zelfs bij het afwezig zijn van lawaai (geluidsniveau kwam niet boven de $60 \mathrm{~dB}$ SPL), zodat de functie van de SM ook goed past in de interferentie (demaskeren) en anti-over-stimulatie theorieën. Deze geluidsmodulerende functies van de SM en de verhoogde gevoeligheid voor NIHL die mogelijk door verlies van de SM functie na routine otosclerose chirurgie wordt veroorzaakt, zijn een reden om te streven naar het behoud van een functionerende SM tijdens de chirurgische behandeling van otosclerose. 
- 
Appendix 1

\section{Clinical application of stapedius muscle contraction}


In daily audiological clinical practice the activity of the stapedius muscle (SM) is used as a diagnostic tool. SM contraction is part of the acoustic reflex (AR) when it is elicited in response to a loud sound. The $A R$ is used clinically in the following cases.

\section{Ossicular chain abnormalities}

Recording the AR in a patient provides information about the mobility of the stapes or the integrity of the ossicular chain. The AR is absent when the stapes is fixed as in otosclerosis or when the ossicular chain is interrupted.

\section{Assessment of hearing level}

Acoustic reflex threshold (ART) measurements have been proposed as a way to determine the hearing level in neonates (Kankkunen and Liden, 1984), or in malingering patients or in patients in which not a reliable tone audiogram can be obtained. ART measurement has been shown to effectively detect hearing losses exceeding $30 \mathrm{~dB}$ in adults. Furthermore, in patients with sensorineural hearing loss (SNHL), the term that covers hearing loss caused by dysfunction of the cochlea, the auditory nerve or of the entire auditory nervous system, the threshold of the AR is more increased for broadband noise than for tones (Margolis, 1993). Determination in a patient of the ART for broadband noise predicts best for average loss of hearing (Hyde et al., 1980).

In patients with SNHL measuring the amplitude of the AR with increasing stimulus intensity can help to differentiate between cochlear dysfunction and auditory nerve dysfunction (retrocochlear). In retrocochlear dysfunction an increased latency and smaller amplitude of the AR is found (Møller, 2000). The failure of persistence of the AR response on prolonged stimulation, i.e. the reflex decay test is specifically associated with auditory nerve lesions (Hirsch, 1983).

In patients suffering from hyperacusis whereby acoustic stimuli provoke unpleasant and painful sensations the loudness discomfort level (LDL) is decreased. In these patients ascertainment of an intact $A R$ is needed to exclude peripheral causes for hyperacusis, such as facial nerve palsy or myasthenia gravis, before considering the possibility of central causes like demyelinating lesions in the central auditory pathway (Weber et al., 2002).

\section{Hearing aid fitting}

The perception of loudness and especially the sound pressure level (SPL) at which the loudness discomfort is experienced, i.e. the LDL is supposed to have a relation with the ART. ART determination in conjunction with measurements of hearing threshold level makes an accurate estimate of LDL possible (Charuhas et al., 1978). In sensorineural hearing-impaired individuals the ART can predict LDL accurately enough to be used clinically as a tool for hearing aid fitting in patients incapable of communicating their acoustic sensation. Investigators successfully predicted LDL within $+/-10 \mathrm{~dB}$ for a high percentage of the subjects (McLeod and Greenberg, 1979; Sanborn, 1998). In normal hearing individuals the ART not 
always turned out to be a valid predictor of individual loudness perception (Knobel and Sanchez, 2006; Morgan et al., 1979; Olsen et al., 1999a). In cochlear-impaired listeners a high correlation of the ART with the most comfortable listening level was found (Olsen et al., 1999b). The results indicated that the most comfortable listening level and AR fell at approximately the same intensities under all test conditions, whereas the LDL occurred at approximately $18 \mathrm{~dB}$ higher intensity (Keith, 1979).

\section{Cochlear implantation}

Cochlear implantation $(\mathrm{Cl})$ includes placing of an electrode in the cochlea that can electrically

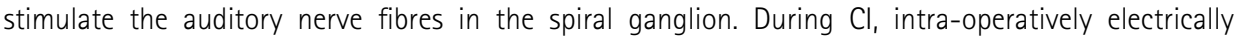
elicited ART can be used to predict maximum comfortable levels to improve speech processor fitting postoperatively (Gordon et al., 2004; Zehnder et al., 1999). The electrically elicited AR does provide a means to estimate an approximate LDL.

\section{Neurological diseases}

The AR could serve as a prognostic indicator of cochlear recovery in sudden onset SNHL (Gerwin and LaCoste, 1982) and of facial nerve recovery in Bell's palsy cases (Portmann et al., 1990). This information is also useful in the topodiagnosis in facial nerve paralysis patients; the presence or absence of an AR indicates a lesion distal or proximal of branching of the stapedial nerve, respectively (Ide et al., 1988). Furthermore the AR in patients with untreated myasthenia gravis was found to be typically depressed or absent and was used in monitoring the effects of therapy (Bischoff et al., 1989; Neff et al., 1980; Toth et al., 2000).

\section{References}

- Bischoff, C., Klingelhofer, J., Conrad, B., 1989. Decay and recovery of the stapedial reflex by prolonged stimulation in the diagnosis of myasthenia gravis. J. Neurol. 236(6), 343-348.

- $\quad$ Charuhas, P.A., Chung, D.Y., Barry, S., 1978. Relationship between uncomfortable loudness level and acoustic reflex threshold as a function of hearing loss. J. Aud. Res. 18(4), 237-242.

- Gerwin, J.M., LaCoste, P., 1982. The acoustic stapedial reflex as a prognostic indicator in sudden onset sensorineural hearing loss. Otolaryngol. Head Neck Surg. 90(6), 857-861.

- Gordon, K.A., Papsin, B.C., Harrison, R.V., 2004. Toward a battery of behavioral and objective measures to achieve optimal cochlear implant stimulation levels in children. Ear Hear. 25(5), 447-463.

- Hirsch, A., 1983. The stapedius reflex tests in retrocochlear hearing disorders. Audiology 22(5), 463-470.

- Hyde, M.L., Alberti, P.W., Morgan, P.P., Symons, F., Cummings, F., 1980. Pure tone threshold estimation from acoustic reflex thresholds-a myth? Acta Otolaryngol. 89(3-4), 345-357.

- Ide, M., Morimitsu, T., Ushisako, Y., Makino, K., Fukiyama, M., Hayashi, A., 1988. The significance of stapedial reflex test in facial nerve paralysis. Acta Otolaryngol. Suppl. 446, 57-63.

- Kankkunen, A., Liden, G., 1984. Ipsilateral acoustic reflex thresholds in neonates and in normal-hearing and hearing-impaired pre-school children. Scand. Audiol. 13(2), 139-144. 
- Keith, R.W., 1979. Loudness and the acoustic reflex: cochlear-impaired listeners. J. Am. Audiol. Soc. 5(2), 65-70.

- Knobel, K.A., Sanchez, T.G., 2006. Loudness discomfort level in normal hearing individuals. Pro Fono. 18(1), 31-40.

- Margolis RH. 1993. Detection of hearing impairment with the acoustic stapedius reflex. Ear Hear.14 (1), 3-10.

- McLeod, H.L., Greenberg, H.J., 1979. Relationship between loudness discomfort level and acoustic reflex threshold for normal and sensorineural hearing-impaired individuals. J. Speech Hear. Res. 22(4), 873-883.

- Møller, A.R., 2000. Hearing: Its Physiology and Pathophysiology, section III; Acoustic reflexes, Academic Press, San Diego, CA, pp. 364-365.

- Morgan, D.E., Dirks, D.D., Bower, D., Kamm, C.A., 1979. Loudness discomfort level and acoustic reflex threshold for speech stimuli. J. Speech Hear. Res. 22(4), 849-861.

- Neff, P.A., Morioka, W.T., Sample, P.A., Cantrell, R.W., 1980. Audiometric and tympanometric monitoring of a disease affecting nerve-muscle transmission. Audiology 19(4), 293-309.

- Olsen, S.O., Rasmussen, A.N., Nielsen, L.H., Borgkvist, B.V., 1999a.The acoustic reflex threshold: not predictive for loudness perception in normally-hearing. Audiology 38(6), 303-307.

- Olsen, S.O., Rasmussen, A.N., Nielsen, L.H., Borgkvist, B.V., 1999b. The relationship between the acoustic reflex threshold and levels of loudness categories in hearing-impaired listeners. Audiology 38(6), 308-311.

- Portmann, M., Dauman, R., Negrevergne, M., Cazenave, M., 1990. The prognostic value of the stapedius reflex in peripheral facial palsy. Ear Nose Throat J. 69(10), 696-697.

- Sanborn, P.E., 1998. Predicting hearing aid response in real ears. J. Acoust. Soc. Am. 103(6), 3407-3417.

- Toth, L., Lampe, I., Dioszeghy, P., Repassy, G., 2000. The diagnostic value of stapedius reflex and stapedius reflex exhaustion in myasthenia gravis. Electromyogr. Clin. Neurophysiol. 40(1), 17-20.

- Weber, H., Pfadenhauer, K., Stohr, M., Rosler, A., 2002. Central hyperacusis with phonophobia in multiple sclerosis. Mult. Scler. $8(6), 505-509$.

- Zehnder, A., Allum, J.H., Honegger, F., Probst, R., 1999. Bedeutung der intraoperativ registrierten elektrischen Stapediusreflexschwelle für die Cochlear-implant-Anpassung bei Kindern. HNO 47(11), 970-975. 
Appendix 2

Muscle fibre types; their classification and properties 


\section{Introduction}

The performance of a skeletal muscle depends on the contractile properties of its fibres; we assume that this is also the case for the SM. Muscle fibre properties as contraction velocity and fatigue resistance are dependent on their myosin heavy chain ( $\mathrm{MHC}$ ) isoform protein contents. The $\mathrm{MHC}$ isoforms can be considered as molecule markers of a complete pattern of protein expression including for example, the enzymes involved in the production of ATP. The pattern of MHC isoforms is the molecular equivalent of the fibre type for determination of the muscle function as determent by physiology (Bottinelli et al., 1999). It is therefore the main marker used in our investigation concerning the function of the SM.

\section{Skeletal muscle anatomy}

Skeletal muscles consist of bundles of muscle fibres, which are elongated multinuclear cells that are orientated more or less in the line of muscle action. The myofibrils in the muscle fibre accommodate the actual contractile elements. The myofibrils are formed by continuous chains of sarcomeres. The muscle fibres are called striated due to their appearance under the light microscope which is caused by the regular arrangement of the myofibrils such that the parallel running sarcomeres are aligned perpendicular in the line of muscle fibre action. The contraction of a muscle is the resultant of an orchestrated action of the sarcomeres, the basic units of muscle function. The sarcomere is formed by an arrangement of the proteins actin and myosin in filaments that interact resulting in sliding movement in relation to each other (Fig. A2.1).

This process of sliding filaments involves the forming of temporary cross-bridges between the myosin and actin molecules. The contractile force arises from a large conformational change in the attached head of the myosin molecule, either in the binding angle made with actin (Huxley, 1969) or within the head itself (Rayment et al., 1993; Burgess et al., 1997). For cross-bridge cycling to occur, Mg-ATP and $\mathrm{Ca} 2+$ must be present. Sarcomere length (and therefore the extent of overlap between thin and thick filaments) affects the number of cross-bridges present. This is the fundamental basis of the forcelength relationship. The myosin heavy chain $(\mathrm{MHC})$ is a structural protein component of the large myosin molecule (Fig. A2.2). It is also an enzyme (ATPase) which enzymatically hydrolyses adenosine triphosphate (ATP) and is therefore the key factor in determining the nature of excitation-contraction coupling and movement (Barany, 1967).The energy from each cycle of cross-bridge formation comes from splitting of ATP into ADP and Pi.

Besides myosin and actin there are many other proteins characterized in the sarcomere which contribute to the contractile processes. These include tropomyosin, troponin $T_{1}$ troponin $I$, troponin $C, C$ protein, $H$ protein, M protein, capZ, titin, $\alpha$-actinin, nebulin, and others, which have been reviewed by Schiaffino and Reggiani, (1996).

Myosin is a large protein molecule composed of two MHCs and four myosin light chains (MLC). The MLC can be divided into two groups, the alkali or essential chains and the regulatory light chains (Fig. A2.2). The exact role of the myosin light chains in muscle contraction is still uncertain. In rat limb muscle the 


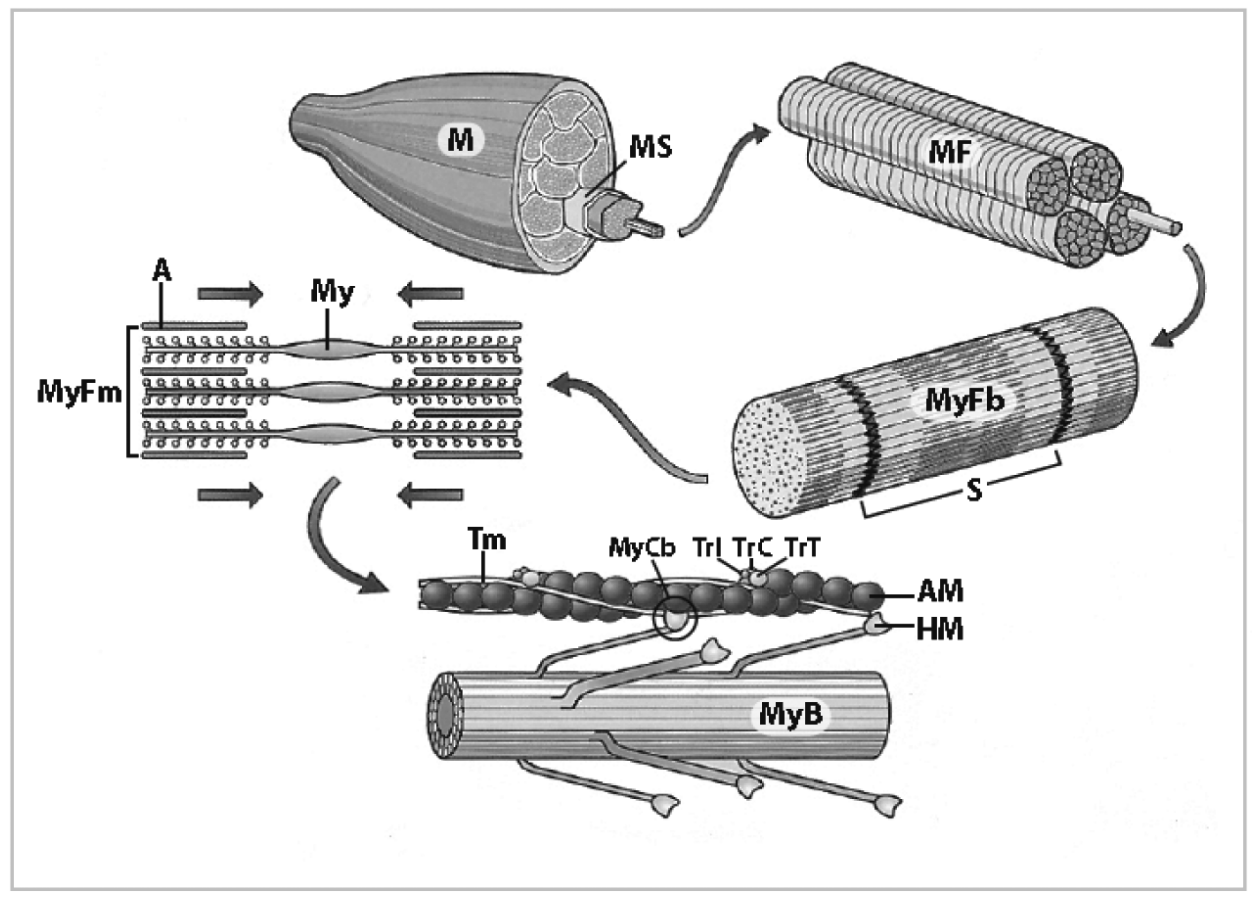

Fig. A2.1. Skeletal muscle organisation. A, actin (thin filament); $A M$, actin molecule; $H M$, head of myosin molecule; $M$, muscle; $M F$, muscle fibre (cell); MS, muscle segment (fasciculus); My, myosin (thick filament); MyB, myosin backbone; MyCb (in circle), myosin cross-bridge; MyFb, myofibril; MyFm, myofilaments; S, sarcomere; Tm, tropomyosin; $\operatorname{TrC}$, troponin C; Trl, troponin l; TrT, troponin T.

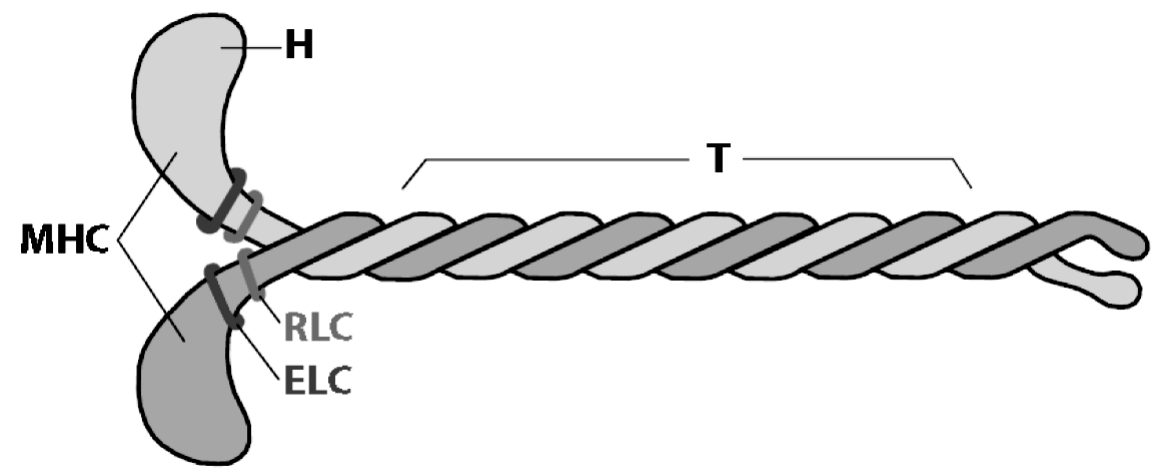

Fig. A2.2. Myosin molecule. ELC, essential myosin light chain; $H$, head of molecule; MHC, myosin heavy chain; $R L C$, regulatory myosin light chain; T, tail of molecule. 
MLCs appear to modify the shortening speed of muscle fibres with some type MHC II in their myosin (Bottinelli et al., 1994), but in human skeletal muscle this effect has not been demonstrated (Larsson and Moss, 1993). The MLC isoform content varies within fibre types. Generally fast contracting fibre types (type II fibres) contain fast regulatory MLC isoform and different proportions of the fast essential MLC isoforms, slow contracting (type I) fibres contain slow regulatory MLC isoform and slow essential MLC isoform. Slow MLC isoforms were also present in type IIA fibres (Andruchov et al., 2006). It is generally accepted that while the cross-bridge kinetics are mainly determined by the MHC isoforms present, it can be affected by a mixture of fast and slow MLC isoforms. The physiological role of fast and slow MLC isoforms in type IIA fibres is a fine-tuning of the cross-bridge kinetics (Andruchov et al., 2006).

In mammalian skeletal muscle nine MHC isoforms have been identified. Embryonic and neonatal MHC isoforms are expressed during muscle development. In terms of MHC gene expression, both slow and fast muscles are still in an undifferentiated state at the time of birth; the embryonic and neonatal MHC isoforms are abundantly expressed shortly after birth. During the first 3-4 weeks of neonatal development, both slow and fast muscles rapidly grow and differentiate into their adult MHC phenotype (d'Albis et al., 1989; Di Maso et al., 2000).

Typically, there are four myosin heavy chain ( $\mathrm{MHC}$ ) isoforms expressed in adult skeletal muscles: one slow type designated as type $\mathrm{MHCI}$ (or cardiac- $B$ when addressed in the cardiac muscle) and three fast types, designated as MHC IIA, IIX, and IIB. Other MHCs have been described; super fast IIM, extraocular (EO) myosin which is named IIL MHC also, being the main MHC in the posterior cricoarytenoid muscle, are expressed in only a few specialized muscles (Wieczorek et al., 1985; Perie et al., 2000). The cardiac- $\alpha$ MHC isoform is expressed in the masseter muscle of the adult rat (Bredman et al., 1991; 1992). These proteins come from a large gene family. All of the genes for the skeletal muscle MHC proteins have been characterized by cDNA and/or genomic cloning, and are found in two chromosomal clusters (Mahdavi et al., 1984; Weiss and Leinwand, 1996).

Much physiological information can be gathered about a muscle by a systematic classification of its fibres. Through the years, in a large number of different species, comparison of muscle fibre type characteristics between different skeletal muscles has been conducted using a variety of techniques. These techniques include: myofibrillar ATPase activity assessed by histochemical staining; histochemical staining to determine metabolic enzyme levels or more recently techniques that involve the determination of MHC by means of immunohistochemistry.

\section{Fibre typing by enzymehistochemistry (EHC)}

Barany (1967) demonstrated that if myosin is extracted from skeletal muscle and subsequently activated in the presence of actin, the amount of ATPase activity produced is directly proportional to the speed of shortening of the muscle from which the myosin was taken, the notion of fast and slow myosin isoforms was established. Myofibrillar ATPase (mATPase) staining methods were developed which determine sarcomeric mATPase activity only. Brooke and Kaiser found that the ATPase activity of the different isoforms was $\mathrm{pH}$ dependent. Pre-incubating muscle in acid or alkali buffers before histochemical 
ATPase activity staining could distinguish between types I, IIA and IIB fibres (Brooke and Kaiser, 1970). Modifications of the mATPase assays were numerous (Guth and Samaha, 1969; Ogilvie and Feeback, 1990; Schiaffino et al., 1989) and the double pre-incubation technique enables identification of a new myosin type IIX (Gorza, 1990; Sant'Ana Pereira et al., 1995).

It was recognized that the ability of a muscle to resist fatigue is associated with the oxidative capacity of muscle fibres. Skeletal muscle fibres which need large amounts of ATP rapidly for short periods show a high activity of glycolytic enzymes, e.g. $\alpha$-glycerophosphate dehydrogenase (GPD). A muscle fibre classification system that combined the mATPase activity staining quality with the activity assays of glycolytic as well as oxidative enzymes, resulted in fibre types like slow oxidative (SO), fast oxidative glycolytic (FOG) and fast glycolytic (FG) (Peter et al., 1972). To sustain the high workload imposed by the innervating nerve, slow fibres have a high oxidative capacity and are therefore not easily fatigued. In skeletal muscles of mouse, rat, and rabbit amongst the fast type fibres, type IIB fibres are the largest, with a high glycolytic capacity and these are the least oxidative fibres. The type IIA fibres are the smallest with intermediate to high levels of glycolytic enzymes and these are the most oxidative fibres. Type IIX fibres are intermediate to these values (Hämäläinen and Pette, 1993).

\section{Fibre typing by immunohistochemistry $(\mathrm{IHC})$}

Fibre type-specific antibodies with reactivity for individual isoforms of MHC were developed. The ability to electrophoretically separate isoforms of MHC enabled the development of these antibodies. Numerous different antibodies were generated in various species, against numerous domains of the myosin molecules. The poor electrophoretic resolutions of $\mathrm{MHC}$ isoform bands are probably due to the high degree of molecular homology, 78-98\% amino acid identity between myosin isoforms in striated muscles (Weiss and Leinwand, 1996). Antibodies specific for type I, IIA and IIB were produced first, and supported the earlier histochemical classification system. The third major type II MHC, type IIX, and its presence in some muscles has extended the histochemical classification system (DeNardi et al., 1993; Gorza, 1990; Sant'Ana Pereira et al., 1995). In human muscles it was revealed that the actual MHC isoform designated earlier as IIB in mATPase type IIB fibres is in fact the MHC isoform IIX. Although the MHC IIB gene has been identified in the human genome, evidence for its expression at the protein level has not been established (Sant'Ana Pereira et al., 1997; Smerdu et al., 1994). It is likely that earlier histochemical studies misclassified the type IIX fibre as type IIB, as well in human as in animal studies. The large differences in shortening velocity and energy consumption among the members of the myosin family appear to be solely due to variations in the rates and equilibrium constants of the acto-myosin interaction cycle (the cross-bridge cycle) and not to the existence of different molecular mechanisms (Capitanio et al., 2006). The reported comparable shortening speeds of adult skeletal myosins is extraocular $>I I M>I I B>I I X>I I A>$ cardiac- $\alpha>$ I (Schiaffino and Reggiani, 1996; Sciote and Kentish, 1996). The phenotypic differences in contractile, metabolic, and morphological properties between fibre types are related with MHC content. Fibres expressing MHC IIX and/or MHC IIB generate greater force, but the ATP consumption per cross bridge cycle is much higher than in fibres expressing $\mathrm{MHC}$ I or MHC IIA 
isoforms, the latter two are the most energy efficient (Han et al. , 2003; He et al., 2000 ). Slow fibres have the lowest mATPase activity (related to shortening velocity), the highest oxidative capacity, the lowest glycolytic metabolism and glycogen content, the smallest cross-sectional area (CSA), the greatest capillary, and nuclear densities, the reverse pattern is true for pure IIB fibres, whereas type IIA and IIX fibres have intermediate properties. Discrimination of muscle fibres according to their MHC content was possible on the basis of their contractile and non-contractile profiles (Quiroz-Rothe and Rivero, 2004).

\section{MHC polymorphism}

IHC on serial muscle tissue cross-sections and single fibre analysis has revealed the phenomenon of MHC hybridism or polymorphism; single muscle fibres co-expressing more than one MHC isoform in various combinations. Neither of the EHC methods traditionally employed to distinguish fibre types in skeletal muscles (mATPase-based or metabolic enzyme-based histochemistry) can be used effectively for the identification and characterization of MHC hybrids (Hämäläinen and Pette, 1995; Schiaffino and Reggiani, 1996). The frequency of their occurrence in various muscles and species suggests that hybrid fibres are not a rare phenomenon. Hybrid fibres are present in both developing and adult muscles, also in transforming skeletal muscle in response to changes of innervation or altered usage. Skeletal muscles also show adaptations in MHC-isoform composition in response to decrease or increase of neuromuscular activity (Demirel et al., 1999; Schuler and Pette, 1996). Permanent MHC hybridism of adult muscle fibres could serve a smooth but possibly relative fast adaptation to new functional changing demands (Baldwin and Haddad, 2001; Caiozzo et al., 2000; Caiozzo et al., 2003).

In MHC hybrid fibres a continuum of muscle fibre properties, such as maximum shortening velocity (Vmax), oxidative capacity, glycolytic enzyme activity and muscle fibre CSA is displayed (Caiozzo et al., 2003; Pette and Staron, 2000; Rivero et al., 1998; Staron and Pette, 1993; Stephenson, 2001). The correlation between the MHC isoform content of single fibres and their CSA, succinate dehydrogenase (SDH) and $\alpha$-glycerophosphate dehydrogenase (GPD) values in the rat medial gastrocnemius muscle was consistent enough that most of the fibres could be discriminated into discrete groups with the same MHC isoforms content when their CSA, SDH and GPD values were considered together (Rivero et al., 1998). Hybrid fibres (in porcine muscles) have mean values of mATPase activity (related to shortening velocity), SDH activity (oxidative capacity), GPD activity (glycolytic metabolism), glycogen content, CSA, capillary and nuclear densities, intermediate in between their respective pure phenotypes at both extremes pure type MHC I and type MHC IIB (Quiroz-Rothe and Rivero, 2003). The prevailing view is that hybrid fibres enable a muscle to fine-tune its efficiency for the wide range of forces, velocities, levels of endurance and levels of resistance to fatigue it is required to generate (Pette and Staron, 2000; Pette, 2002; Stephenson, 2001).

\section{Postnatal development of mammalian muscles}

The mammalian skeletal muscle development involves a transition from dominance of developmental Emb MHC and Neo MHC isoform expression towards adult MHC isoforms. Towards a 
more adult age a transient shift into a muscle specific defined adult MHC phenotype occurs (Agbulut et al. 2003). Studies on postnatal development of mammalian muscles showed programs of myosin expression for each muscle specifically, the sequential transition of myosin isoforms during postnatal development is controlled by multiple factors such as functional demand, innervation, calcium influx and hormonal signals (Schiaffino and Reggiani, 1996; Pette and Staron, 2000; Watchko et al., 1998). The contraction velocity of developmental Emb MHC and Neo MHC isoform seems to be slow (D'Antona et al., 2003). The speed of the transition towards adult MHC isoforms reflects the importance of mature functioning of specific muscles soon after birth (d'Albis et al., 1989; Butler-Browne and Whalen, 1984; Lloyd et al., 1996; Sutherland et al., 1993; Usami et al. 2003; Wang et al., 2004).

\section{Adaptation to the functional demands of the muscle}

Adaptation of muscle phenotype was described on the level of enzymes involved in pathways of ATP production (Chillibeck et al., 1998; Tikkanen et al., 1995) or involves decrease or increase in muscle mass (Chalmers et al., 1992). Increase in mitochondrial content serves to enhance the aerobic-oxidative capacity of energy supply for sustained performance, while fast-to-slower fibre-type transitions, induced by exercise, form the basis for more economical energy expenditure (Pette and Staron, 2000). After reduced neuromuscular activity, a shift towards fast $\mathrm{MHC}$ isoforms at the expense of the slow MHC I usually occurs, with the magnitude of this shift varying among the different animal and experimental models used (Talmadge, 2000).

That metabolic and contractile properties may be co-ordinately regulated follows from work on the calcineurin-induced upregulation of slow-fibre type-specific myofibrillar protein isoforms and metabolic properties in case of increased muscle use (Chin et al. 1998; Olson and Williams, 2000; Meissner et al. 2001). The $\mathrm{Ca}^{2+}$-calmodulin protein kinase-related pathway also induces both metabolic (mitochondrial biogenesis) and myofibrillar adaptations (Allen and Leinwand, 2002). An independent pathway of signal transduction involving AMP-activated protein kinase has been identified as being related to only the metabolic adaptation to increased contractile activity such as exercise training or chronic low frequency stimulation (CLFS) (Hutber et al. 1997; Putman et al. 2003; Winder et al., 2006). Enhanced contractile activity creates the intracellular conditions of increased 5 -AMP and $\mathrm{Ca}^{2+}$ concentrations that are both necessary and sufficient to simultaneously activate all three pathways, and probably accounts for the apparent co-ordinated regulation of metabolic changes with MHC-based fibre type transitions typically observed after exercise training and CLFS (Hood et al., 2006; Pette and Staron, 2000; Pette and Vrbová, 1992).

\section{Muscle fibre typing in the stapedius muscle}

The muscle fibres of the SM have been characterized in different species (Anniko and Wroblewski, 1981; Burgener and Mayr, 1980; Lyon and Malmgren, 1982; Mascarello et al., 1983; Veggetti et al., 1982; Berge and Wirtz 1989; Godfrey et al., 1990a; Godfrey et al., 1990b; de Jong, 1988; Vita et al., 1983). There exists no data on the human SM fibre composition. Different staining protocols and muscle 
fibre classification methods have been used leading to a variety of description of the fibre types. Results of these investigations are summarized in Table A1.

Table A1.

Literature on histochemical characterized SM fibres in various animal species

1 Guinea pig: Combined electron microscopy and mATPase methods; two thirds of the fibres resembled myotubes and stain for mATPase like IIC fibres, which suggested a developing nature. One third of the fibres appeared to be more mature, they stained like type I fibres. (Burgener and Mayr, 1980).

2 Guinea pig: Combined electron microscopy and mATPase methods; the muscle fibres were multinucleated, contained a high amount of ribosomes, a centriole was often found adjacent to the cell nucleus. Both type I and type II fibres occurred, the latter with three subtypes (Anniko and Wroblewski, 1981).

3 Horse, sheep, cow, pig, dog, cat and rabbit: enzymehistochemistry; Type I and IIA fibres in ratios of 20/80\% up to $50 / 50 \%$, presumably belonging to slow and fast contracting fatigue-resistant motor units. Fast-twitch white fibres type IIB were not found (Veggetti et al., 1982).

4 Cat: enzymehistochemistry; $77 \%$ fast oxidative glycolytic fibres (FOG, supposed to be equivalent to type IIA) and 23\% smaller fibres, 13\% type 1' fibres and 10\% type 2' fibres, assumed to be comparable to slow oxidative (SO) type I and fast glycolytic (FG) type IIB (Lyon and Malmgren, 1982).

5 Cat, dog, fox, ferret, stone-marten, New World monkeys (Callithrix, Saimiri) and Old World monkeys (Cercopithecus, Macaca); mATPase based type I (slow-twitch) and IIA fibres, Immunohistochemically determined slow-tonic fibres were found in the SMs of only two carnivores, the ferret and stone-marten (Mascarello et al., 1983)

6 Rabbit: enzymehistochemistry by means of the myofibrillar ATPase and NADH-tetrazolium reductase techniques; two different fibre types, type 1 and type 2b, were identified in the SM. (Vita et al., 1983)

7 Rat: enzymehistochemistry, mATPase, SDH and GPD straining; IIA fibres (87\%) and slow fibres (5\%), both staining in a moderate to strong way to SDH as well as GPD. The fast twitch fibres were classified type IIA, because of their high oxidative as well as glycolytic capacity (immunohistochemistry showed discrepancies with enzymehistochemistry; first time mention of MHC hybridism) (van den Berge and Wirtz, 1989).

8 Cat SM and rat SM homogenates: High activities of the enzymes choline acetyltransferase and acetylcholinesterase. High activity of oxidative enzymes in rat SMs, low activity in the cat SM. Glycolytic enzyme activity in rat and cat was remarkably low. (Godfrey et al., 1990 a; Godfrey et al., 1990 b).

9 Rat SM homogenates: MHC IIX (58.0\%+/- 4.2\%) and MHC IIA (32.3\%+/- 6.7\%), with a smaller percentage of MHC IIB $(7.4 \%+/-0.2 \%)$ and $\mathrm{MHCI}(1.9 \%+/-0.1 \%)$. Neonatal and embryonic MHCs were detected at very low levels (Jung et al., 2004). 


\section{References}

- $\quad$ Agbulut, O., Noirez, P., Beaumont, F., Butler-Browne, G., 2003. Myosin heavy chain isoforms in postnatal muscle development of mice. Biol. Cell. 95(6), 399-406

- d'Albis, A., Couteaux, R., Janmot, C., Roulet, A., 1989. Specific programs of myosin expression in the postnatal development of rat muscles. Eur. J. Biochem. 183(3), 583-590.

- $\quad$ Allen, D.L., Leinwand, L.A., 2002. Intracellular calcium and myosin isoform transitions. Calcineurin and calcium-calmodulin kinase pathways regulate preferential activation of the lla myosin heavy chain promoter. J. Biol. Chem. 277(47), 45323-45330.

- $\quad$ Andruchov, O., Andruchova, O., Wang, Y., Galler, S., 2006. Dependence of cross-bridge kinetics on myosin light chain isoforms in rabbit and rat skeletal muscle fibres. J. Physiol. 571, 231-242.

- Anniko, M., Wroblewski, R., 1981. Ultrastructure and elemental composition of the stapedius muscle in guinea pig. Arch. Otorhinolaryngol. 230(2), 109-112.

- $\quad$ Baldwin, K.M., Haddad, F., 2001. Plasticity in Skeletal, Cardiac, and Smooth Muscle, Invited Review: Effects of different activity and inactivity paradigms on myosin heavy chain gene expression in striated muscle. J. Appl. Physiol. 90, 345-357.

- Barany, M., 1967. ATPase activity of myosin correlated with speed of muscle shortening, J. Physiol., 213, 455-474.

- $\quad$ Berge, H. van den, Wirtz, P., 1989. Detailed morphology of the stapedius muscle of the rat. J. Anat. 166, 157-169.

- $\quad$ Bottinelli, R., Betto, R., Schiaffino, S., Reggiani, C., 1994. Unloaded shortening velocity and myosin heavy chain and alkali light chain isoform composition in rat skeletal muscle fibres. J. Physiol. 478(2), 341-349.

- Bottinelli, R., Pellegrino, M.A., Canepari, M., Rossi, R., Reggiani, C., 1999. Specific contributions of various muscle fibre types to human muscle performance: an in vitro study. J. Electromyogr. Kinesiol. 9(2), 87-95.

- $\quad$ Bredman, J.J., Wessels, A., Weijs, W.A., Korfage, J.A., Soffers, C.A., Moorman, A.F., 1991. Demonstration of cardiac-specific myosin heavy chain in masticatory muscles of human and rabbit. Histochem. J. 23, 160-170.

- Bredman, J.J., Weijs, W.A., Moorman, A.F., 1992. Presence of cardiac $\alpha$-myosin correlates with histochemical myosin Ca2+ ATPase activity in the rabbit masseter muscle. Histochem. J. 24, 260-265.

- $\quad$ Brooke, M.H., Kaiser, K.K., 1970. Muscle fibre types how many and what kind? Arch. Neurol. 23, 369-379.

- $\quad$ Burgener, J., Mayr, R., 1980. Guinea pig stapedius muscle. A histochemical, light and electron microscopic study. Anat. Embryol. $161,65-81$.

- $\quad$ Burgess, S.A., Walker, M.L., White, H.D., Trinick, J., 1997. Flexibility within myosin heads revealed by negative stain and singleparticle analysis. J. Cell Biol. 139(3), 675-681.

- $\quad$ Butler-Browne, G.S., Whalen, R.G., 1984. Myosin isozyme transitions occurring during the postnatal development of the rat soleus muscle. Dev. Biol. 102(2), 324-334.

- $\quad$ Caiozzo, V.J., Haddad, F., Baker, M., McCue, S., Baldwin, K.M., 2000. MHC polymorphism in rodent plantaris muscle: effects of mechanical overload and hypothyroidism. Am. J. Physiol. Cell Physiol. 278, C709-C717.

- $\quad$ Caiozzo, V.J., Baker, M.J., Huang, K., Chou, H., Wu, Y.Z., Baldwin, K.M., 2003. Single-fiber myosin heavy chain polymorphism: how many patterns and what proportion? Am. J. Physiol. Regul. Integr. Comp. Physiol. 285(3), R570-R580.

- Capitanio, M., Canepari, M., Cacciafesta, P., Lombardi, V., Cicchi, R., Maffei. M., Pavone, F.S., Bottinelli, R., 2006. Two independent mechanical events in the interaction cycle of skeletal muscle myosin with actin. Proc. Natl. Acad. Sci. 103(1), 87-92.

- Chalmers, G.R., Roy, R.R., Edgerton, V.R., 1992. Variation and limitations in fiber enzymatic and size responses in hypertrophied muscle. J. Appl. Physiol. 73 (2), 631-641. 
- Chin, E.R., Olson, E.N., Richardson, J.A., Yang, Q., Humphries, C., Shelton, J.M., Wu, H., Zhu, W., Bassel-Duby, R., and Williams, R.S. 1998. A calcineurin-dependent transcriptional pathway controls skeletal muscle fiber phenotype. Genes Develop. 12, 24992509 .

- Chillibeck, P.D., Bell, G.J., Socha, T., Martin, T., 1998. The effect of aerobic exercise training on the distribution of succinate dehydrogenase activity throughout muscle fibres. Can. J. Appl. Physiol. 23 (1), 74-86.

- D'Antona, G., Pellegrino, M.A., Adami, R., Rossi, R., Carlizzi, C.N., Canepari, M., Saltin, B., Bottinelli, R., 2003. The effect of ageing and immobilization on structure and function of human skeletal muscle fibres. J. Physiol. 552, 499-511.

- Demirel, H.A., Powers, S.K., Naito, H., Hughes, M., Coombes, J.S., 1999. Exercise-induced alterations in skeletal muscle myosin heavy chain phenotype: dose-response relationship. J. Appl. Physiol. 86(3), 1002-1008.

- DeNardi, C., Ausoni, S., Moretti, P., Gorza, L., Velleca, M., Buckingham, M., Schiaffino, S., 1993. Type 2X-myosin heavy chain is coded by a muscle fiber type-specific and developmentally regulated gene. J. Cell. Biol. 123(4), 823-835.

- $\quad$ Di Maso, N.A., Caiozzo, V.J., Baldwin, K.M., 2000. Single-fiber myosin heavy chain polymorphism during postnatal development: modulation by hypothyroidism. Am. J. Physiol. Regul. Integr. Comp. Physiol. 278(4), R1099-R1106.

- Gorza, L., 1990. Identification of a novel type 2 fibre population in mammalian skeletal muscle by combined use of histochemical myosin ATPase and anti-myosin monoclonal antibodies. J. Histochem. Cytochem. 38, 257-265.

- Godfrey, D.A., Judkins, R.F., Wiet, G.J., Parli, J.A., Ross, C.D., Rubin, A.M., 1990a. Enzymes of transmitter and energy metabolism in cat middle ear muscles. Otolaryngol. Head Neck Surg.103, 799-804.

- Godfrey, D.A., Wiet, G.J., Parli, J.A., Beranek, K.L., Ross, C.D., 1990b. Enzymes of transmitter and energy metabolism in rat middle ear and extraocular muscles. Hear. Res. 48(3), 187-194.

- Guth, L. and Samaha, F.J., 1969. Procedure for the histochemical demonstration of actomyosin ATPase. Exp. Neurol. 28, 365367.

- Hämäläinen, N., Pette, D., 1993. The histochemical profiles of fast fiber types IIB, IID, and IIA in skeletal muscles of mouse, rat, and rabbit. J. Histochem. Cytochem. 41(5), 733-743.

- Hämäläinen, N., Pette, D., 1995. Patterns of myosin isoforms in mammalian skeletal muscle fibres. Microsc. Res. Tech. 30(5), 381-389.

- Han, Y.S., Geiger, P.C., Cody, M.J., Macken, R.L., Sieck, G.C., 2003. ATP consumption rate per cross bridge depends on myosin heavy chain isoform. J. Appl. Physiol. 94(6), 2188-2196.

- He, Z.H., Bottinelli, R., Pellegrino, M.A., Ferenczi, M.A., Reggiani, C., 2000. ATP consumption and efficiency of human single muscle fibers with different myosin isoform composition. Biophys. J. 79(2), 945-961.

- Hood, D.A., Irrcher, I., Ljubicic, V., Joseph, A.M., 2006. Coordination of metabolic plasticity in skeletal muscle. J. Exp. Biol. 209(Pt 12), 2265-2275.

- Hutber, C.A., Hardie, D.G., Winder, W.W., 1997. Electrical stimulation inactivates muscle acetyl-CoA carboxylase and increases AMP-activated protein kinase. Am. J. Physiol. 272(2 Pt 1), 262-266.

- Huxley, H.E., 1969. The mechanism of muscular contraction. Science 164(886), 1356-1365.

- Jong, F.I.C.R.S. de, Kingma, H., Wirtz, P., Berge, H. van den, Marres, E.H.M.A., 1988. Indications of a differentiated regulation of sound transmission by the middle ear muscles of the rat. Am. J. Otol. 9(1), 70-75.

- Jung, H.H., Han, S.H., Nam, S.Y., Kim, Y.H., Kim, J.L., 2004. Myosin heavy chain composition of rat middle ear muscles. Acta Otolaryngol. 124(5):569-573. 
- Larsson, L., Moss, R.L., 1993. Maximum velocity of shortening in relation to myosin isoform composition in single fibres from human skeletal muscles. J. Physiol. 472, 595-614.

- $\quad$ Lloyd, J.S., Brozanski, B.S., Daood, M., Watchko, J.F., 1996. Developmental transitions in the myosin heavy chain phenotype of human respiratory muscle. Biol. Neonate 69(2), 67-75.

- Lyon, M.J., Malmgren, L.T., 1982. A histochemical characterisation of muscle fiber types in the middle ear muscles of the cat, the stapedius muscle. Acta Otolaryngol. 94, 99-109.

- Mahdavi, V., Chambers, A.P., Nadal-Ginard, B., 1984. Cardiac alpha- and beta-myosin heavy chain genes are organized in tandem. Proc. Natl. Acad. Sci., 81(9), 2626-2630.

- Mascarello F, Veggetti A, Carpene E, Rowlerson A., 1983. An immunohistochemical study of the middle ear muscles of some carnivores and primates, with special reference to the IIM and slow-tonic fibre types. J. Anat. 137, 95-108.

- Meissner, J.D., Gros, G., Scheibe, R.J., Scholz, M., Kubis, H.P., 2001. Calcineurin regulates slow myosin, but not fast myosin or metabolic enzymes, during fast-to-slow transformation in rabbit skeletal muscle cell culture. J. Physiol. 533, 215-226.

- Ogilvie, R.W., Feeback, D.L., 1990. A metachromatic dye-ATPase method for the simultaneous identification of skeletal muscle fibre types I, IIA, IIB and IIC. Stain Technol. 65, 231-241.

- Olson, E.N., Williams, R.S., 2000. Calcineurin signalling and muscle remodelling. Cell 101(7), 689-692.

- $\quad$ Perie, S., Agbulut, O., St Guily, J.L, Butler-Browne, G.S., 2000. Myosin heavy chain expression in human laryngeal muscle fibers. A biochemical study. Ann. Otol. Rhinol. Laryngol. 109(2), 216-220.

- Peter, P.B., Barnard, R.J., Edgerton, V.R., 1972. Metabolic profiles of three fibre types of skeletal muscle in Guinea pigs and rabbits. Biochemistry 11, 2627-2633.

- $\quad$ Pette, D., 2002. The adaptive potential of skeletal muscle fibers. Can. J. Appl. Physiol. 27(4), 423-448.

- Pette, D., Staron, R.S., 2000. Myosin isoforms, muscle fiber types, and transitions. Microsc. Res. Tech. 50(6), 500-509.

- Pette, D., Vrbová, G., 1992. Adaptation of mammalian skeletal muscle fibers to chronic electrical stimulation. Rev. Physio. Biochem. Pharmacol. 120, 115-202.

- $\quad$ Putman, C.T., Kiricsi, M., Pearcey, J., MacLean, I.M., Bamford, J.A., Murdoch, G.K., Dixon, W.T., Pette, D., 2003. AMPK activation increases uncoupling protein-3 expression and mitochondrial enzyme activities in rat muscle without fibre type transitions. J. Physiol. 551,169-178.

- Quiroz-Rothe, E., Rivero, J.L., 2004. Coordinated expression of myosin heavy chains, metabolic enzymes, and morphological features of porcine skeletal muscle fiber types. Microsc. Res. Tech. 65(1-2), 43-61.

- $\quad$ Rayment, I., Holden, H.M., Whittaker, M., Yohn, C.B., Lorenz, M., Holmes, K.C., Milligan, R.A., 1993. Structure of the actin-myosin complex and its implications for muscle contraction. Science 261(5117), 58-65.

- $\quad$ Rivero, J.L., Talmadge, R.J., Edgerton, V.R., 1998. Fibre size and metabolic properties of myosin heavy chain-based fibre types in rat skeletal muscle. J. Muscle Res. Cell Motil. 19, 733-742.

- Sant'Ana Pereira, J.A.A., Haan, de A., Wessels, A., Moorman, A.F.M., Sargeant, A.J., 1995. The mATPase histochemical profile of rat type IIX fibres: Correlation with MyHC immunolabelling. Histochem. J. 27, 715-722.

- Sant'Ana Pereira, J. A., Ennion, S., Sargeant, A. J., Moorman, A. F. and Goldspink, G., 1997. Comparison of the molecular, antigenic and ATPase determinants of fast myosin heavy chains in rat and human: a single-fibre study. Pflügers Arch. 435, 151-163.

- Schiaffino, S., Gorza, L., Sartore, S., Saggin, L., Ausini, S, Vianello, M., Gundersen, K., Lomo, T., 1989. Three myosin heavy chain isoforms in type 2 skeletal muscle fibres. J. Muscle Res. Cell Motil. 10, 197-205. 
- Schiaffino, S., Reggiani, C., 1996. Molecular diversity of myofibrillar proteins: gene regulation and functional significance. Physiol. Rev. 76, 371-423.

- Schuler, M., Pette, D., 1996. Fiber transformation and replacement in low-frequency stimulated rabbit fast-twitch muscles. Cell Tissue Res. 285(2), 297-303.

- Sciote, J.J., Kentish, J.C., 1996. Unloaded shortening velocities of rabbit masseter muscle fibres expressing skeletal or alphacardiac myosin heavy chains. J. Physiol. 492, 659-667.

- Smerdu, V., Karsch-Mizrachi, I., Campione, M., Leinwand, L., Schiaffino, S., 1994. Type Ilx myosin heavy chain transcripts are expressed in type Illb fibers of human skeletal muscle. Am. J. Physiol. 267, C1723-C1728.

- Staron, R.C., Pette, D., 1993. The continuum of pure and hybrid myosin heavy chain based fibre types in rat skeletal muscles. Histochemistry 100, 149-153.

- Stephenson, G.M., 2001. Hybrid skeletal muscle fibres: a rare or common phenomenon? Clin. Exp. Pharmacol. Physiol. 28(8), 692-702.

- Sutherland, C.J., Esser, K.A., Elsom, V.L., Gordon, M.L., Hardeman, E.C., 1993. Identification of a program of contractile protein gene expression initiated upon skeletal muscle differentiation. Dev. Dyn. 196(1), 25-36.

- Talmadge, R., J., 2000. Myosin heavy chain isoform expression following reduced neuromuscular activity: potential regulatory mechanisms. Muscle Nerve 23 (5), 661-679.

- Tikkanen, H.O., Naveri, H.K., Harkonen, M.H., 1995. Alteration of regulatory enzyme activities in fast-twitch and slow-twitch muscles in low intensity endurance trained rats. Eur. J. Appl. Occup. Physiol. 70 (4), 281-287.

- Usami, A., Abe, S., Ide, Y., 2003. Myosin heavy chain isoforms of the murine masseter muscle during pre- and post-natal development. Anat. Histol. Embryol. 32(4), 244-248.

- Veggetti, A., Mascarello, F., Carpene, E., 1982. A comparative histochemical study of fibre types in middle ear muscles. J. Anat. $135,333-352$.

- Vita, G., Muglia, U., Germana, G., Pennica, F., Carfi, F., 1983. Histochemical characteristics of rabbit stapedius muscle. Exp. Neurol. $81(2), 511-516$.

- Wang, J., Han, Y., Su, H., Mu, L., 2004. Expression of unique and developmental myosin heavy chain isoforms in adult human digastric muscle. J. Histochem. Cytochem. 52(7), 851-859.

- Watchko, J.F., Daood, M.J., Sieck, G.C., 1998. Myosin heavy chain transitions during development. Functional implications for the respiratory musculature. Comp. Biochem. Physiol. Biochem. Mol. Biol. 119(3), 459-470.

- Weiss, A., Leinwand, L.A., 1996. The mammalian myosin heavy chain gene family. Ann. Rev. Cell Dev. Biol. 12, 417-439.

- Wieczorek, D.F., Periasamy, M., Butler-Browne, G.S., Whalen, R.G., Nadal-Ginard, B., 1985. Co-expression of multiple myosin heavy chain genes, in addition to a tissue-specific one, in extraocular musculature. J. Cell. Biol. 101(2), 618-629.

- Winder, W.W., Taylor, E.B., Thomson, D.M., 2006. Role of AMP-activated protein kinase in the molecular adaptation to endurance exercise. Med. Sci. Sports Exerc. 38(11), 1945-1949. 


\section{Dankwoord}

En dan is het proefschrift er eindelijk. De stroomversnelling waarin het onderzoek van de stapedius spier de afgelopen 2 jaren terecht is gekomen, staat in contrast met de trage vorderingen van de jaren ervoor. Het leek wel een mission impossible. Het realiseren van dit proefschrift heb ik te danken aan een groot aantal mensen. De volgende personen wil ik op deze plaats in het bijzonder noemen.

Prof. dr. H. van Mameren, beste Henk. Ik ben je dankbaar voor je nimmer aflatende structurele steun aan het onderzoek, ondanks dat het onderzoek zelf niet meer paste in de dagelijkse praktijkvoering van je afdeling. Het bij mij afdwingen van een logische manier van denken en schrijven over de materie heeft er toe geleid dat het onderzoek en het hieruit voortkomende proefschrift betekenis heeft gekregen. Een vriendschap is gesmeed.

Prof. dr. J.J. Manni, beste Hans. Ik ben je dankbaar voor je belangstelling in de vorderingen van het onderzoek, juist ook in perioden dat er relatief weinig nieuws te melden was. Je scherpzinnige blik is diverse malen over conceptteksten heen gegaan waardoor de leesbaarheid en inhoud steeds beter werden.

P. van Dijk, zoölogisch analist, beste Paul. Vanaf het prille begin van dit onderzoek ben jij mijn steun en toeverlaat geweest. We hebben samen uitgezocht welke histochemische technieken we het beste gebruiken konden. Ondanks het bezoeken van andere universitaire laboratoria, wij toch zelf moesten uitvinden hoe en onder welke condities het spiertje behandeld diende te worden. De vele uren van coupes snijden, kleuren en fotograferen zijn jou ten deel gevallen, het werk wat je hebt verricht met de $1.2 \mathrm{~mm}$ grote spiertjes mag een huzarenstuk worden genoemd. Het is ook aan jouw doorzettingsvermogen en trouw te danken dat de publicaties er zijn gekomen en ook uiteindelijk dit boek is verschenen.

Dr. L.J.C. Anteunis, beste Lucien. Jouw inbreng was veel meer dan de feitelijke bijdrage aan de hoofdstukken. Je altijd ingetogen en nuchtere kijk op zaken heeft bijgedragen aan het afronden van het onderzoek wat natuurlijk ook altijd de bedoeling is geweest.

Michelène Chenault, beste Mickey. Dank voor je statistische analyses en taalkundige correcties. Veel dank voor de fijne samenwerking.

Prof. dr. E.H.M.A. Marres, beste Ed. Nu het proefschrift af is, wil ik je danken voor de opdracht die je mij destijds hebt gegeven om te starten met het onderzoek naar de functie van de stapedius spier. Bovenal dank ik je nog voor de opleidingsplaats tot KNO-arts in Maastricht en voor het feit dat ik op jouw voordracht een jaar ervaring heb mogen opdoen bij Professor Stell in Liverpool. 
Prof. dr. J. Drukker, u stond mede aan de wieg van de samenwerking tussen de KNO en de Anatomie/ Embryologie afdeling te Maastricht. Veel dank voor de interesse en tijd die $u$ aan het beoordelen van mijn publicaties in wording heeft besteed.

Hans Rensema, wetenschappelijk illustrator en vormgever, veel dank voor het bewerken van figuren en het ter beschikking stellen van je eigen kunst ten behoeve van dit boekje.

De medewerkers van de vakgroep Anatomie en Embryologie van de Universiteit Maastricht, bedankt voor de gastvrijheid en belangstelling.

letje Thiery, hartelijk dank voor het ontwerp van de omslagillustratie en de voorbereidingen van de layout van de thesis.

Professor dr. A.F.M. Moorman, bedankt voor het beschikbaar stellen van de antilichamen en voor de gewaardeerde medewerking aan de eerste publicatie die als beschrijving van materiaal en methode een belangrijke basis van deze thesis is.

Ook de overige druk bezette leden van de beoordelingscommissie wil ik hartelijk danken voor het kritisch doorlezen van het manuscript.

Michel Oude Ophuis, paranimf en maatschapslid, beste Michel. Jouw morele steun is er altijd geweest, waarvoor dank. Je hebt vele uren gestoken in het redigeren van teksten vanuit mijn steenkool Engels tot leesbare literatuur. Ik ben je hier erg dankbaar voor.

Mijn andere maten, Welty Elprana en Eric Theunissen, jullie hebben achter iedere keuze gestaan met betrekking tot dit onderzoek. We zijn straks een "zeergeleerde maatschap". Beste Eric, Michel en Welty, ik hoop met jullie, als maatschap KNO-artsen van het VieCuri, nieuwe uitdagingen aan te mogen gaan in de komende jaren en onze fantastische praktijk verder te ontwikkelen.

Rob Brouwer, goede vriend en paranimf, beste Rob. Je vriendschap en je altijd positieve steun hebben zeker bijgedragen aan het afronden van mijn promotieonderzoek. Het was voor mij vanzelfsprekend jou te vragen mij ook tijdens de openbare verdediging van deze thesis te steunen, ook al ligt je expertise op het juridische vlak. Ik kan me geen betere paranimf voorstellen.

Lieve pa en ma, schoonouders, familie en vrienden, diverse malen is de voortgang van mijn onderzoek bij gelegenheid voorzichtig ter sprake gekomen en ik wist dat ik kon vertrouwen op jullie steun. Het heeft even geduurd maar zie hier, het resultaat. 
Mijn schatten van kinderen, Floris, Maud en Joost, jullie hebben vooral de lasten gehad van pa's onderzoek, jullie hebben het te vaak zonder mij moeten doen. Ik beloof de komende jaren de vrije tijd meer met jullie in te vullen, waar ik me trouwens ook op verheug. Jullie zijn mijn inspiratie, ik zal mijn best doen dat ook voor jullie te blijven.

Ten slotte, lieve Petra. Niemand anders dan jij weet beter wat het kost om te leven met iemand met een onvoltooide missie als een promotieonderzoek. Jou komt dan ook een groot deel van de eer toe dat het afgekomen is, en dat terwijl het gezin floreert. Schat, ik hou van je.

Patrick F.M. Dammeijer,

Venlo, november 2007 